

\section{LIGHT IN CONTROL OF TWISTING MATTER}

Supitchaya Iamsaard 
Members of the committee:

Chairman: Prof. dr. W.P.M. van Swaaij (University of Twente)

Promotors: Prof. dr. N. Katsonis (University of Twente)

Prof. dr. J.J.L.M. Cornelissen (University of Twente)

Members: Prof. dr. D.J. Broer (Eindhoven University of

Technology)

Prof. dr. S. P. Fletcher (University of Oxford)

Prof. dr. J. Huskens $\quad$ (University of Twente)

Dr. M. Hempenius $\quad$ (University of Twente)

The research described in this thesis was performed within the laboratories of the Biomolecular Nanotechnology (BNT) group, the MESA+ institute for Nanotechnology, and the Department of Science and Technology (TNW) of the University of Twente. This research was supported by the Netherlands Organization for Scientific Research (NWO) and by the European Research Council (ERC).

\section{MESA+ \\ INSTITUTE FOR NANOTECHNOLOGY}

UNIVERSITY OF TWENTE.

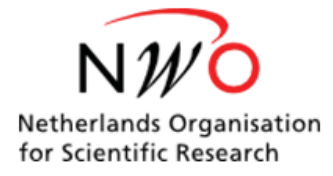
for Scientific Research

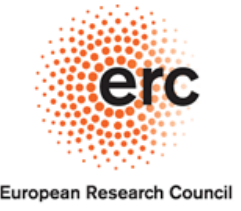

European Research Council

\section{Light in control of twisting matter}

Copyright (C) 2016, Supitchaya Iamsaard, Enschede, The Netherlands.

All rights reserved. No part of this thesis may be reproduced or transmitted in any form, by any means, electronic or mechanical without prior written permission of the author.

ISBN: $\quad 978-90-365-4069-8$

DOI: $\quad 10.3990 / 1.9789036540698$

Cover art: $\quad$ PANCAT (Pichamon Graphic House), Supitchaya Iamsaard

Printed by: $\quad$ Gildeprint Drukkerijen - The Netherlands 


\title{
LIGHT IN CONTROL OF TWISTING MATTER
}

\author{
DISSERTATION
}

\author{
to obtain \\ the degree of doctor at the University of Twente, \\ on the authority of rector magnificus \\ Prof. dr. H. Brinksma,
}

On account of the decision of the graduation committee,

to be publicly defended

on Friday March 4, 2016 at 14.45 h

by

\author{
Supitchaya Iamsaard \\ Born on May 24, 1985 \\ in Samutsongkhram, Thailand
}


This dissertation has been approved by:

Promotors: Prof. dr. Nathalie Katsonis

Prof. dr. Jeroen J.L.M. Cornelissen 
This thesis is dedicated to my family 


\section{Table of contents}

$\begin{array}{ll}\text { Chapter 1: Introduction } & 1\end{array}$

1.1 General Introduction 1

$\begin{array}{lll}1.2 & \text { Liquid crystals and liquid crystal polymer networks } & 1\end{array}$

1.3 Aim and outline 2

1.4 References 4

Chapter 2: Strategies to amplify the light-driven motion of 5 molecules up to the macroscopic level

2.1 Introduction 6

$\begin{array}{lll}2.2 & \text { Nanoscale mechanics of photochromic molecules } & 7\end{array}$

2.2.1 Translational mode 7

2.2.2 Rotational mode 8

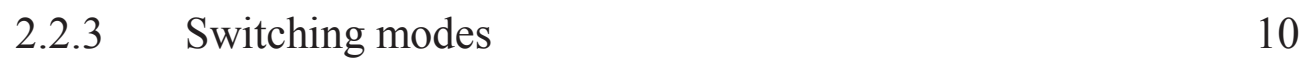

2.2.4 Other complex mechanical modes 11

2.3 Amplification of molecular mechanics in polymer networks $\quad 14$

$\begin{array}{lll}\text { 2.3.1 Light-responsive polymer gels } & 15\end{array}$

2.3.2 Light-responsive liquid crystal polymer networks 18

$2.4 \quad$ Macroscopic motion at work 31

2.5 Conclusions 33

$\begin{array}{lll}2.6 & \text { References } & 34\end{array}$

Chapter 3: Bio-inspired strategies to convert light into 39 macroscopic twist motion

$\begin{array}{lll}3.1 & \text { Introduction } & 40\end{array}$

3.2 Results and discussion $\quad 40$

3.2.1 Amplifying molecular chirality in a liquid crystal 40 polymer

3.2.2 Generating a diversity of helical shapes 42

3.2.3 Photoactuation modes of the helical ribbons 46

3.2.4 Mimicking the mechanical behaviour of plant tendrils $\quad 50$ 
3.3 Conclusions $\quad 52$

3.4 Acknowledgments 53

3.5 Materials and methods 53

$\begin{array}{lll}3.5 .1 & \text { General } & 53\end{array}$

3.5.2 Synthesis of the molecular switch $\quad 54$

3.5.3 Preparation of the springs, structural characterization $\quad 55$ and photo-mechanical properties

$\begin{array}{ll}3.5 .4 & \text { Supplementary information }\end{array}$

$\begin{array}{lll}3.6 & \text { References } & 62\end{array}$

Chapter 4: Preserving the delayed relaxation of $o$-fluorinated 65 azobenzenes in liquid crystal polymer networks

$\begin{array}{lll}4.1 & \text { Introduction } & 66\end{array}$

$\begin{array}{lll}4.2 & \text { Results and discussion } & 69\end{array}$

4.2.1 Photochemistry of F-1 in solution 69

4.2.2 Photochemistry of $\mathbf{F - 1}$ once covalently bound to the 70 liquid crystal polymer network

4.2.3 Photo-actuation and shape relaxation 72

$\begin{array}{lll}4.3 & \text { Conclusions } & 75\end{array}$

$\begin{array}{lll}4.4 & \text { Acknowledgments } & 76\end{array}$

$\begin{array}{lll}4.5 & \text { Materials and methods } & 76\end{array}$

$\begin{array}{lll}4.5 .1 & \text { General } & 76\end{array}$

4.5.2 Synthesis of F-1 76

4.5.3 Preparation of the ribbons 77

$\begin{array}{lll}\text { 4.5.4 Supplementary information } & 78\end{array}$

$\begin{array}{lll}4.6 & \text { References } & 79\end{array}$

Chapter 5: Twisting matter with light: engineering photo- 83 switchable topological states

$\begin{array}{lll}5.1 & \text { Introduction } & 84\end{array}$

$\begin{array}{lll}5.2 & \text { Results and discussion } & 85\end{array}$

$\begin{array}{lll}5.3 & \text { Conclusions } & 92\end{array}$

5.4 Acknowledgments 92

$\begin{array}{lll}5.5 & \text { Materials and methods } & 92\end{array}$ 
5.5.1 General 92

5.5.2 Synthesis and characterization of the chiral dopants 93

5.5.3 Preparation of the frustrated films of light-responsive 94

5.6 References

96

Chapter 6: Towards dual-responsive liquid crystal films

6.1 Introduction

100

$\begin{array}{lll}\text { 6.2 } & \text { Results and discussion } & 101\end{array}$

6.2.1 A dual-responsive cholesteric liquid crystal and its 101 photo-response under confinement

6.2.2 Creation of localized defects in thin films of dual 106 responsive liquid crystals using light

6.2.3 Creation of localized defects in thin films of dual 108 responsive liquid crystals using electric field

$\begin{array}{lll}\text { 6.3 Conclusions and perspectives } & 109\end{array}$

$\begin{array}{lll}6.4 & \text { Acknowledgments } & 109\end{array}$

$\begin{array}{lll}\text { 6.5 } & \text { Materials and methods } & 109\end{array}$

6.5.1 General 109

6.5.2 Preparation of the cholesteric liquid crystals 110

6.5.3 Helical twisting power of dopants in a dual frequency 111

6.6 References

113

Summary

115

$\begin{array}{ll}\text { Samenvatting } & 117\end{array}$

$\begin{array}{lr}\text { Acknowledgments } & 119\end{array}$

$\begin{array}{ll}\text { About the author } & 125\end{array}$ 


\section{Chapter 1}

\section{Introduction}

\subsection{General Introduction}

The high-tech materials we will design and engineer in the coming years will have a major impact in shaping our future. Arguably, a sustainable future will require the development of responsive, adaptable, self-healing and maybe even intelligent materials, that all fall under the category of smart materials: materials that are designed to perform a specific function when being commanded, by adjusting their physical and/or chemical properties. ${ }^{1}$

The recent years have seen the development of a variety of stimuli-responsive

materials ${ }^{2-4}$ that respond the external stimuli such as heat, light, magnetic field, electric field, stress and humidity. In the research presented here, we favor the use of light as an external trigger, in view of the high advantages of light technology: clean, rapid and precise stimulus.

The exquisite sophistication of organic chemistry has allowed to develop a broad range of molecules that respond with complex changes to irradiation with UV light. ${ }^{5}$ However, transducing these effects from the molecular level up to the supramolecular and eventually the macroscopic level of smart materials, remains an unrequited challenge.$^{6-9}$ In this thesis, we address the challenge by combining the sensitivity of liquid crystals with the dynamic properties of light-responsive molecular motors and switches.

\subsection{Liquid crystals and liquid crystal polymer networks}

We choose liquid crystals as basic elements to build up smart function due to the high sensitivity of their organization to external stimuli. ${ }^{10,11}$

We design smart materials with special mechanical functions by converting lightresponsive liquid crystals into liquid crystal polymer networks. These liquid crystal polymer networks change shape under irradiation with UV light and allow reaching beyond the state of the art in the stimuli-induced deformation of smart materials, in particular in terms of 
General introduction

versatility and complexity. Moreover, we show that an appropriate design of the photochromic switches and their rational incorporation in the liquid crystal polymer network, allows controlling the kinetics of shape relaxation, once light is switched off.

We also design smart materials with special optical functions by designing the confinement conditions for chiral liquid crystals, with a special focus on cholesteric liquid crystals. Cholesteric liquid crystals display a helix-based organization that is characterised by their pitch, i.e. the distance over which the average molecular orientation rotates by $2 \pi .^{12}$ Due to their helical organisation, cholesteric liquid crystals are well-known for their unique optical properties. ${ }^{12}$ Light-responsive chiral liquid crystals, where photo-chromic molecules are incorporated as dopants, are capable of adjusting their helical structure when light is used as a trigger, which leads to the dynamical control over optical properties such as reflection colours, polarization of light and optical imaging. ${ }^{13-16}$ Recently, these special optical properties have been revisited at the local level, as well-defined microstructures created locally, that can also be considered as topological defects, ${ }^{17}$ and show potential for optical applications such as information storage, diffractive gratings and beam-steering devices. While inspiring examples have been reported in the last few years, possible improvements in the field include 1) optimise the photo-control over the film properties in terms of sensitivity, tuning ability and reversibility 2) developing new alternative strategies for the creation of defects 3) manipulating these defects and controlling their dynamic properties.

\subsection{Aim and outline of this thesis}

The aim of this thesis is to engineer light-responsive materials towards innovative designs for controllable mechanical- (Chapter 3-4) and optical- (Chapter 5-6) functions. The strategy is based on incorporating molecular motors and switches in chiral liquid crystals, which provides matter with the capacity to modulate its twisted organization and properties under command of light. For achieving both functions (Chapter 3-6), a key is that amplifying nanoaction of photo-chromic molecules in chiral liquid crystals using light can lead to smart functions of the materials. 
Chapter 2 contains a short literature overview over the mechanics of molecules at the nanoscale, and strategies that have been developed in order to amplify their molecular mechanics up to larger length-scale functions. A special focus is given to previous achievements involving photo-induced mechanical materials, including light-liquid crystal polymer networks that are able to perform work.

Chapter 3 describes new strategies for the amplification of molecular motion towards the complex photo-deformation of springs, inspired by the curling and twisting of plant tendrils. It was also demonstrated that these materials can perform work.

Chapter 4 deals with the kinetics of relaxation of liquid crystal polymer networks, once light is switched off. While the vast majority of networks that have been developed to date initiate their relaxation as soon as irradiation stops, we show that the use of orthofluoroazobenzenes provides a valid alternative, and that the slow relaxation of these molecular switches can be preserved at the macroscopic level.

In contrast with chapters 3 and 4, where smart materials with mechanical properties are discussed primarily, Chapter 5 involves with molecular materials with stimuli-responsive optical functions. Specifically, the chapter describes how to create a class of defects, twistedmicrostructures named torons, by using light as a trigger in thin films of liquid crystals that twist under irradiation with light. Remarkably, we show that incorporating molecular motors in these liquid crystals allows generating a variety of torons, at a much lower energetic cost.

Chapter 6 builds on chapter 5 and describes a strategy to design dual-responsive cholesteric liquid crystals, where an electric field can be used to create the torons, and light can be used in order to manipulate them - for example, to make them rotate, or to control their translation. These dynamic motion will enable modern optical function, particularly, for spontaneous transformation of optical information. 


\subsection{References}

1 Kobayashi, A. T. a. H. Responsive Materials and Methods: State-of-the-Art StimuliResponsive Materials and Their Applications Wiley-Scrivener, 2013.

2 Schattling, P.; Jochum, F. D.; Theato, P. Polym. Chem. 2014, 5, 25.

3 Kelley, E. G.; Albert, J. N. L.; Sullivan, M. O.; Epps, I. I. I. T. H. Chem. Soc. Rev. 2013, 42, 7057.

4 Murphy, E. B.; Wudl, F. Prog. Polym. Sci. 2010, 35, 223.

5 Peplow, M. Nature 2015, 525, 18.

6 Gibson, L. J. J. R. Soc. Interface 2012.

7 Screen, H. R. C. JBSE 2009, 4, 481.

8 Wang, J.-S.; Wang, G.; Feng, X.-Q.; Kitamura, T.; Kang, Y.-L.; Yu, S.-W.; Qin, Q.-H. Sci. Rep. 2013, 3, 3102.

9 Ikeda, T.; Mamiya, J.-i.; Yu, Y. Angew. Chem. Int. Ed. 2007, 46, 506.

10 Fleischmann, E.-K.; Zentel, R. Angew. Chem. Int. Ed. 2013, 52, 8810.

11 Li, Q. Liquid Crystals Beyond Displays: Chemistry, Physics, and Applications; Wiley, 2012.

12 Eelkema, R.; Feringa, B. L. Org. Biomol. Chem. 2006, 4, 3729.

13 Bisoyi, H. K.; Li, Q. Acc. Chem. Res. 2014, 47, 3184.

14 Ma, J.; Xuan, L. Displays 2013, 34, 293.

15 White, T. J.; McConney, M. E.; Bunning, T. J. J. Mater. Chem. 2010, 20, 9832.

16 Aßhoff, S. J.; Iamsaard, S.; Bosco, A.; Cornelissen, J. J. L. M.; Feringa, B. L.; Katsonis, N. Chem. Commun. 2013, 49, 4256.

17 Smalyukh, I. I.; Lansac, Y.; Clark, N. A.; Trivedi, R. P. Nat. Mater. 2010, 9, 139. 


\section{Chapter 2}

\section{Strategies to amplify the light-driven motion of molecules up to the macroscopic level}

Precise, complex, directional and reversible movement is at the heart of numerous functional systems in biology. In chemistry, light-responsive molecules have been developed that convert light into complex motion. However, in most examples, motion is essentially restricted to the nanoscale, which is essentially the molecular scale, due to a lack of cooperative strategies for the efficient amplification of molecular motion. Some pioneering examples involve the use of crystals, polymers or liquid crystals as media promoting cooperative action, and several materials have been reported in which photogenerated mechanical motion has been achieved successfully. In this chapter we review strategies to harness mechanics at the molecular level and to transduce this motion up to the macroscopic scale. Special focus is given to examples where macroscopic motion shows a potential for application by producing work. 
Strategies to amplify the light-driven motion of molecules up to the macroscopic level

\subsection{Introduction}

Molecular motion is the key to designing and producing dynamic materials and devices. ${ }^{1,2}$ The expression can refer to a movement that takes place at molecular level, ${ }^{3}$ or to a larger scale motion that occurs via well-defined molecular constructions. Molecular motion can be amplified to reach the supramolecular level (e.g. in molecular assemblies) ${ }^{4}$ and eventually the macro-scale (by cooperative effects)..$^{5-7}$ The advantages to designing and creating functional materials and devices controlled by motion at the molecular level lie in the possibility to predict and precisely control the motion in response to stimuli. ${ }^{1,2}$ Molecular motion can be generated by triggers of energy, e.g. electric field, heat or light, which induce motion of either the whole molecule or a part of it. In terms of practical use for applications in materials and devices, the non-contact trigger, the possibility of a fast response and the reversibility of irradiation with light are clear assets. ${ }^{8,9}$ Arguably, the most straightforward manner to impart light-responsiveness to a molecular system is to use photochromic molecules, ${ }^{10-13}$ which are capable of reversibly altering their structure under irradiation with light, and in so doing they provide light-induced mechanical motion at the molecular level.

The motion produced by various classes of photochromic molecules is discussed in the following sections, based on their specific mechanics. I then describe the inclusion of photochromic molecules into photo-responsive gels and liquid crystal polymer networks, which are efficient strategies to amplify molecular motion, from the molecular scale up to the macroscopic level. Both photo-responsive gels and liquid crystal polymer networks are smart polymeric materials that have unique properties, in terms of cross-link density, order of network, and swelling capability in a fluid medium. The swelling-deswelling ability of photo-responsive gels makes them advantageous for use in wet conditions. By contrast, photo-responsive liquid crystal polymer networks can be used in applications where dry conditions are desirable. Achievements in applying both materials are also discussed. 


\subsection{Nanoscale mechanics of photochromic molecules}

Photochromic molecules are defined as molecules that can switch between two different isomers under the influence of light. ${ }^{14,15}$ The key to building and controlling macroscopic motion and functionality effectively at the level of materials and devices is understanding and designing a target motion at the molecular level. The motion of photochromic molecules can be broadly divided into four mechanical modes: 1) the translational mode; 2) the rotational mode; 3) the geometrical switching mode; and 4) other complex modes.

\subsubsection{Translational mode}

Translation corresponds to linear motion, and arguably constitutes the simplest mechanical mode, ${ }^{16,17}$ for which rotaxanes constitute an archetypal example. ${ }^{3,18,19}$ Rotaxanes are typically composed of a mobile macrocycle threaded on an axle unit via molecular recognition (Figure 2.1a). The position where the cyclic ring is locked is determined by competitive affinity between two distinct recognition sites on the axle. The highest affinity site can be reversibly tuned by light, and this drives the linear shuttling of the macrocycle.

Berna et al. ${ }^{20}$ reported the light-triggered motion of a rotaxane-based system produced by photo-isomerization of a fumaramide moiety (Figure 2.1b). The trans-cis isomerization of the fumaramide gave rise to a change in the stronger binding affinity of the two, i.e. the fumaramide and fluoroalkane site, yielding a linear shuttling motion. Interestingly, this shuttling is associated with the disclosure or concealment of the fluoroalkane site, which constitutes an asset for designing surfaces with switchable wetting properties. 
a

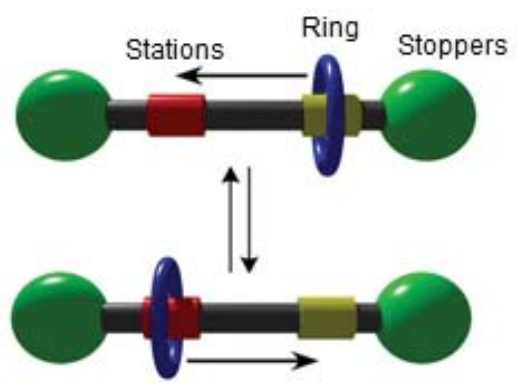

$\mathrm{b}$

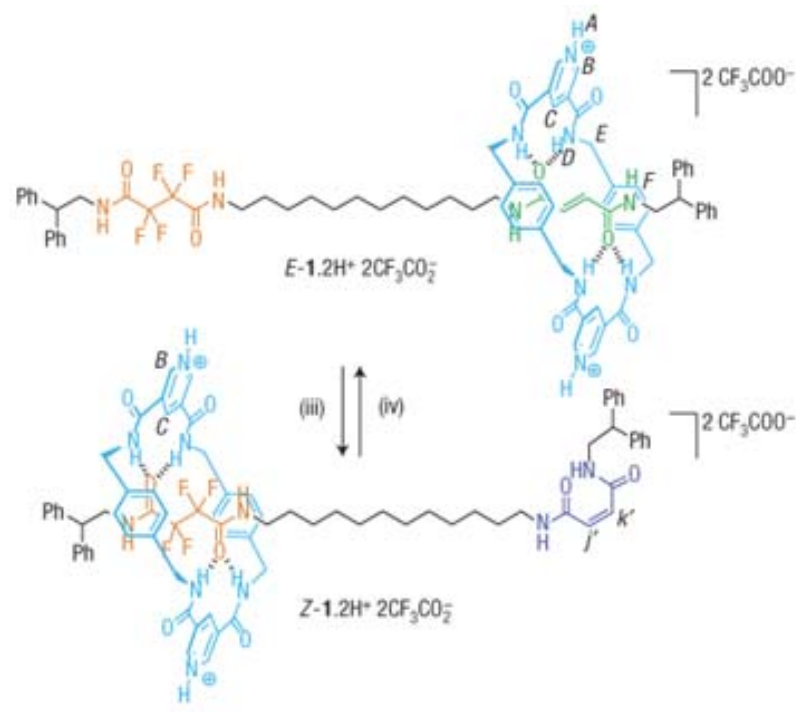

Figure 2.1 a) Model concept of a molecule showing linear motion. Adapted with permission from [3] (Copyright 2015 American Chemical Society). b) Structure and linear shuttling upon light irradiation of a rotaxane based system. Adapted with permission from [20] (Copyright 2005 Nature Publishing Group).

\subsubsection{Rotational mode}

Molecular rotation, described as motion along a specific axis, has also been reported in molecules. ${ }^{1,16}$ In its simplest setting, rotation can be generated by photo-isomerization of a molecule containing a double bond, either a carbon-carbon or a nitrogen-nitrogen double bond. These basically provide in-plane rotation, with no sense of directionality (Figure 2.2a). ${ }^{1}$ Such random rotation is essentially unpredictable and it can cancel out from one molecule to another, leading to poor operation of the molecular device. Feringa et al., however, have designed rotatory motors based on overcrowded alkenes, ${ }^{21,22}$ which demonstrate superior control of directionality by undergoing unidirectional rotation (Figure 2.2b). In these molecular rotors, unidirectionality was imparted by bringing an overcrowded alkene to a higher energy state by trans-cis isomerization, followed by subsequent relaxation to a lower energy state through helix inversion (Figure 2.2c). The energy difference between two helical conformations arises due to the steric strain encoded by a chiral center. These molecular rotors are divided into two mechanical functions: the upper rotor and the lower stator. Upon alternating cycles of excitation with light followed by thermal relaxation, the rotor undergoes a full $360^{\circ}$ rotational motion, which also occurs unidirectionally due to the 
steric impact of the chiral substituent in the so-called "fjord region", as illustrated by the rotary cycle of the first-generation motor 2 (Figure 2.2c). Various designs of a later generation of rotatory motors ${ }^{23}$ have been developed using symmetrical lower halves $\left(2^{\text {nd }}\right.$ generation, Figure 2.2b), and by modifying the size and structure of the substituents in order to control the rotational speed, ${ }^{24,25}$ the wavelength of absorption, ${ }^{26}$ and other properties that must be tuned for specific applications. Further, compound 3, incorporating a second generation motor, was specifically designed to bear tripodal legs and a perfluorobutyl group to permit attachment to a surface and control wettability (Figure $2.2 \mathrm{~d}$ ). ${ }^{27}$

a

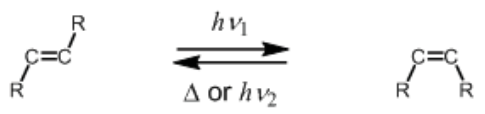

$\mathrm{c}$
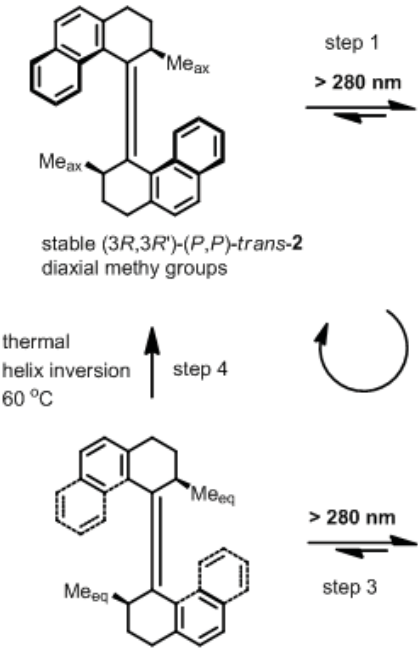

unstable $\left(3 R, 3 R^{\prime}\right)-(M, M)$-trans-2 diequatorial methy groups b
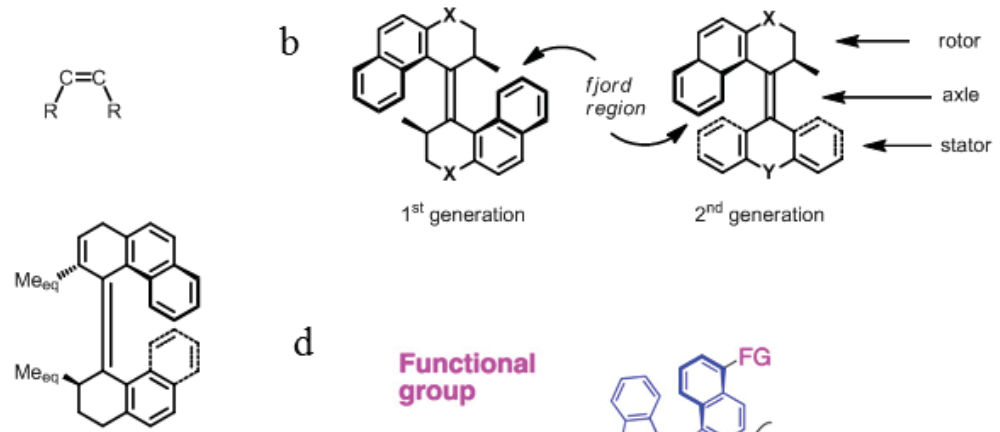

unstable $\left(3 R, 3 R^{\prime}\right)-(M, M)$-cis-2 diequatorial methy groups
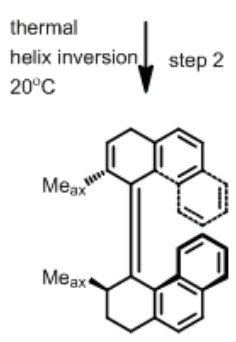

stable $\left(3 R, 3 R^{\prime}\right)-(P, P)$-cis-2 diaxial methy groups

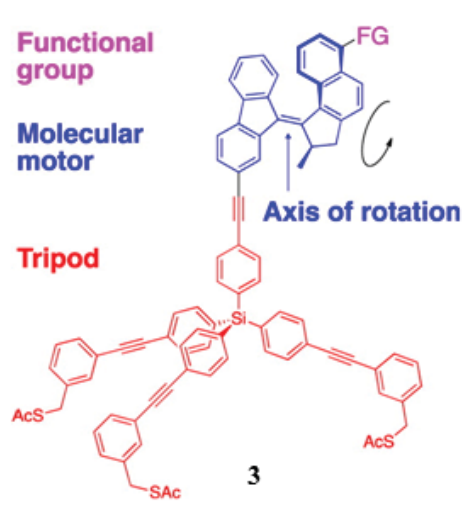

Figure 2.2 a) Light-induced isomerization of alkenes. b) Distinct generational design of overcrowded alkenes capable of demonstrating rotation under light irradiation. c) Lightinduced rotary cycle of $1^{\text {st }}$ generation overcrowded alkene. d) Structure of molecular motor with new design of functional substitution. Adapted with permission from [27] (Copyright 2014 American Chemical Society). 
Strategies to amplify the light-driven motion of molecules up to the macroscopic level

\subsubsection{Switching modes}

Besides translation and rotation, other types of molecular mechanics have also been observed, ${ }^{1}$ as shown in Scheme 2.1. These modes are referred to as switching modes. In these examples, switching takes place via different processes, such as pericyclic reaction, dissociation, intramolecular hydrogen transfers or group transfers, and electron transfer.
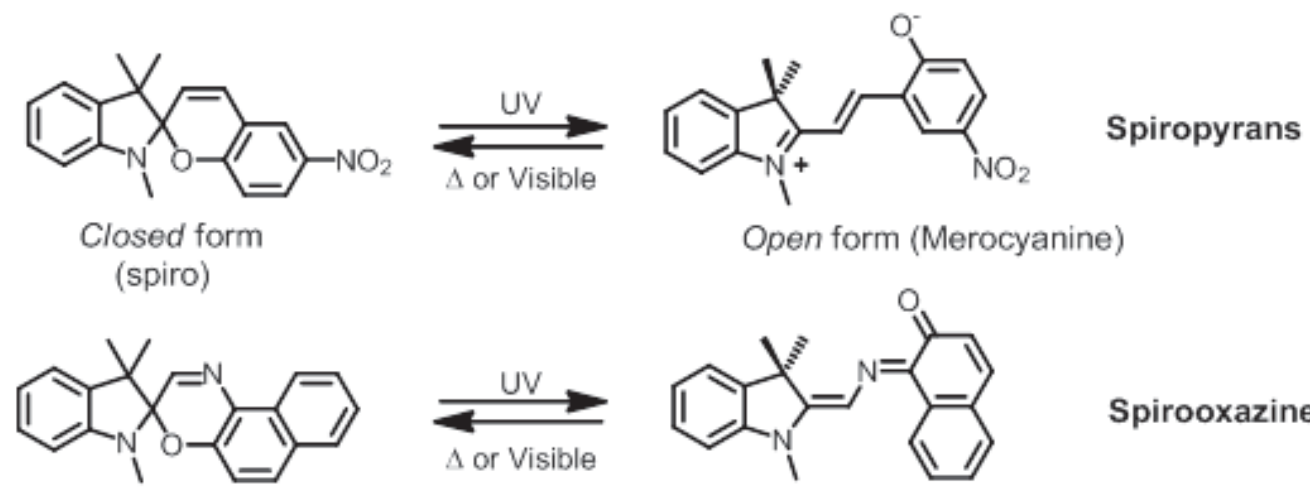<smiles>CN1/C(=C/N=C2C(=O)C=Cc3ccccc32)C(C)(C)c2ccccc21</smiles>

\section{Spirooxazines}

Closed form Open form (Merocyanine)<smiles>Cc1cc2c(/C(=C3\C(C(F)(F)F)C(F)(F)C(F)(F)C3(F)F)c3c(C)sc4ccccc34)cccc2s1</smiles>

Open form<smiles>CC(C)=C1C(=O)OC(=O)C(C)=C1C</smiles>

Open form
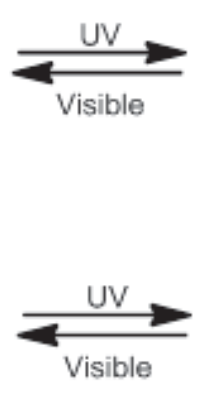

Fulgides

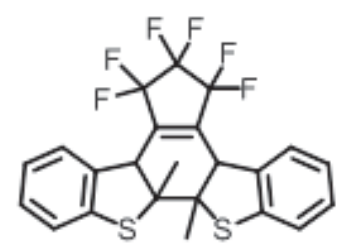

Closed form

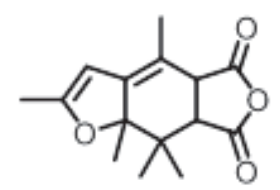

Closed form

Scheme 2.1 Light-induced switching of some photo-active molecules. 


\subsubsection{Other complex mechanical modes}

Complex molecules can yield sophisticated modes of motion, when they are engineered to mimic either the mechanics of macroscopic machines ${ }^{28,29}$ or the complex mechanical modes of biological materials. ${ }^{30,31}$ In these molecular machines, complex actuation modes are built by carefully engineering each molecular component, with a special focus on how the photo-active unit is coupled to the passive moieties of the molecule. Overall, these molecular designs integrate many minor components, among which at least one adopts one of the three basic mechanical modes mentioned above, i.e. linear, rotational, or switching mode. Researchers have designed a large variety of molecular machines, including molecular-tweezers, ${ }^{32-35}$-hinge, ${ }^{36}$-pedal, ${ }^{37,38}$-scissor, ${ }^{28}$ nanocar, ${ }^{39-41}$ and machines displaying clam-like motion ${ }^{30}$ etc. A few examples of sophisticated mechanical modes are discussed below.

Aida and coworkers have successfully created a molecular machine that operates similarly to the opening-closing motion of scissors (Figure 2.3a). ${ }^{28}$ The scissor mechanics were engineered by photo-switching of an azobenzene component (handle, see Figure 2.3b), which in turn gears the in-plane rotation of the ferrocene unit (pivot), and finally transfers to the angular motion of the two connecting phenyl rings (blades). When the azobenzene is in its trans-isomeric form, the great distance between the benzene rings of the azo-unit pulled the phenyl rings at end (A, blade) into an overlapped configuration via a complementary ferrocene motion (pivot), thus causing blade closure. Further, the close proximity of two benzene rings of cis-azobenzene led to the opposite action by photo-isomerization. The scissor motion of molecule 4 was confirmed by CD spectroscopy, which indicated motion change of planar chirality of the ferrocene unit. Moreover, ${ }^{1} \mathrm{H}-\mathrm{NMR}$ displayed an evident down-field shift of the phenyl protons of a blade once opened by light due to reduction of the shielding effect of the phenyl rings as they separate.

Tour and coworkers have constructed a molecular vehicle that incorporates a light-driven molecular motor, ${ }^{39}$ in an effort to propel it under irradiation with light. The nanocar 5 (Figure 2.3c) combines three components: a chassis, two axles, and four wheels that are designed to work together on a surface. Specifically, the idea was to take advantage of the light-driven unidirectional rotation of the molecular motor that would act as a paddle (Figure 2.3d). This 
Strategies to amplify the light-driven motion of molecules up to the macroscopic level

paddle-like rotor is expected to propel nanocar 5 by a force that is generated when interacting with the surface during the rotation. Axles (alkyne with enabling bond rotation) and wheels ( $p$-carborane with near spherical symmetry) are selected to complete the propulsion of nanocar 5. Although photo-induced rotation of the motor unit in $\mathbf{5}$ was achieved with high photo-conversion in solution ( $82 \%$ at photo-stationary state), the slow speed of rotation restricted the propulsion of 5 on a surface. ${ }^{40}$

Interestingly, Feringa et al. have successfully demonstrated propulsion of nanocars 6 along a copper surface with controlled trajectories of motion (Figure 2.3e). ${ }^{42}$ Nanocar 6 is built with four motor units as paddlewheels, of which the concerted unidirectional rotation induced by electrical excitation from the tunneling tips of an STM (scanning tunneling microscope) drive the movement of $\mathbf{6}$ on a surface. The mechanics of the motors in nanocar 6 responding to electrical excitation are similar to excitation by light. Notably, movement of 6 along a surface can be directed to proceed with directionality, at random, or it can be fixed by adjusting the chirality and orientation of each motor unit that is deposited on the surface (Figure 2.3f). 
a

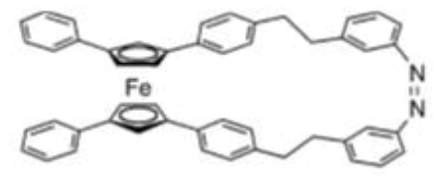

4

$\mathrm{c}$

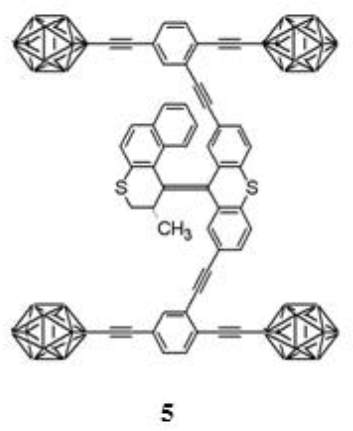

e

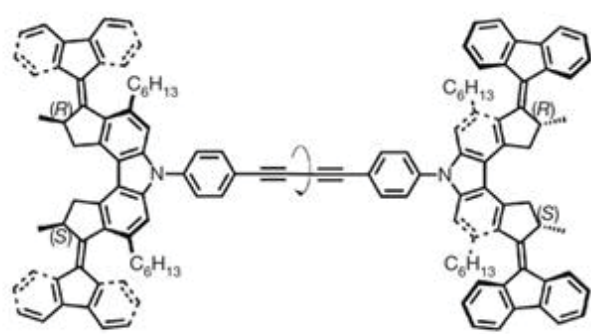

6a (Meso-isomer) b

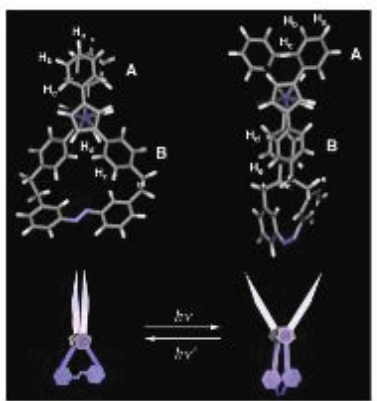

d

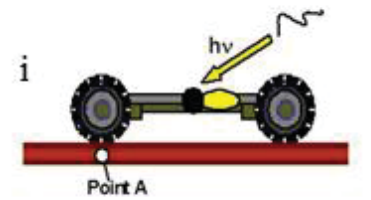

ii

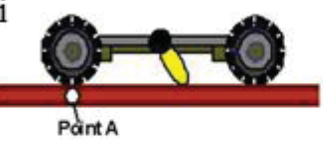

iii

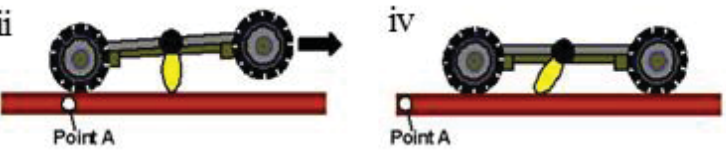

f

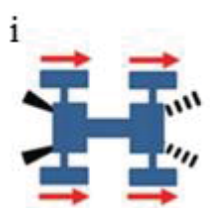

ii

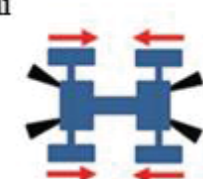

Meso-isomer, 6 a

'correcting landing'

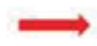

iii

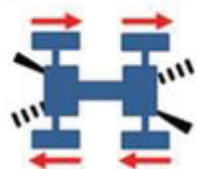

$(R, R-R, R), 6 \mathbf{c}$

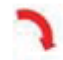

Meso-isomer, $6 \mathrm{~b}$ 'wrong landing'

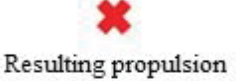

Figure 2.3 a) Design of a molecular scissor and b) representation of its light-induced mechanics. Adapted with permission from [28] (Copyright 2003 American Chemical Society). c) Design of motorized nanocar 5 and d) its expected motion on a surface. Adapted with permission from [39] (Copyright 2006 American Chemical Society). e) Design of nanocar 6a, incorporating four motor wheels. f) Control of motion of $\mathbf{6}$ by distinct chirality and geometry of motor units; i) directional motion; ii) random motion; and iii) immobility. Adapted with permission from [42] (Copyright 2011 Nature Publishing Group).

The demonstration of a range of photo-driven mechanical devices at the molecular scale has paved the way to designing and operating the complex motion of soft functional materials, by transduction of molecular motion up to the supramolecular level ${ }^{4,43}$ and eventually up to the macro-scale, ${ }^{6,7}$ as outlined below. 


\subsection{Amplification of molecular mechanics in polymer networks}

Intuitively, we anticipate that the larger the length scale, the more elusive the amplification of nano-scale motion will be. ${ }^{7}$ In order to achieve motion at the supramolecular level, assembling light-responsive motors or switches via non-covalent interactions is typically sufficient, and has been achieved through $\pi-\pi$ stacking, hydrogen bonding, polar attraction, Van der Waals forces etc. ${ }^{4,44,45}$ For example, Feringa et al. have designed a molecular building block by integrating a dithienylethene photo-switch into hydrophobic cholesterol and hydrophilic poly(ethylene glycol)-modified pyridinium components. ${ }^{44}$ The resulting molecular units form vesicles in water and gel fibers in organic solvents, in which self-assembly is dictated by hydrophobic and hydrophilic interactions with the solvent. Reversible control of the formation of the vesicles and the organogel was achieved upon ringopening and ring-closure of the switching units.

The assembly of photo-active molecules exclusively is also a relevant strategy to build mobile materials, for instance through non-covalent interactions in thin crystals of diarylethenes. ${ }^{46-48}$ However, the macroscopic motion produced by these molecular crystals is usually of low amplitude, because the switches are densely packed, which makes the structure rigid. Moreover, applicability of these systems remains a challenge, since they are brittle and usually crack after deformation, as strain develops within the crystals as a consequence of volume change and phase separation induced by photo-modulation of the switch structure. ${ }^{7}$ The low amplitude of motion and the fragility that characterize thin molecular crystals highlight the inherent restrictions of systems that are composed exclusively of light-responsive molecules.

Designing a sensitive matrix to efficiently amplify the motion of light-responsive switches consequently appears to be a strategic step towards achieving large, reversible, complex, multimodal deformation and motion at the macroscale. Since, by definition, soft matter is sensitive to changes in its environment, polymers and liquid crystals have provided excellent amplification matrices in this regard. ${ }^{5,6,10}$ In polymers, cooperativity originates from the elasticity and mobility of the polymer chains, which allow the network conformation to change, while cooperativity in liquid crystals arises from the high responsiveness of the 
orientational order of the liquid crystal molecules. Below, I describe how the incorporation of light-responsive molecules in polymer gels (section 2.2.1) and liquid crystal polymer networks (section 2.2.2) allows achieving higher efficiencies in the transduction of light into molecular motion, and eventually macroscopic motion.

\subsubsection{Light-responsive polymer gels}

Polymer gels are three-dimensional, cross-linked polymer networks, which can store solvent in large amounts. Typically, in a swollen gel, the ratio of solvent molecules over polymer network reaches up to a hundred times. ${ }^{49,50}$ Polymer gels display relatively great flexibility while beholding the cooperative effects that are key to amplifying molecular motion. Because they can swell, polymer gels are typically used to work in wet conditions. Depending on the type of bonds which are linking the polymer chains in the network, polymer gels can be classified into either chemical gels or physical polymer gels. Physical gels usually display low thermal stability, i.e. they dissolve above a certain temperature. The temperature-dependent behavior of physical gels constitutes an additional challenge in terms of applications, which is why this section focuses primarily on chemical gels that are formed by polymers. Photo-active molecules have been integrated successfully in polymer gels to engineer macroscopic functionality, including motion, shape transformation, and assembly. ${ }^{51-54}$ Conceptually, photo-induced motion of photo-active molecules can lead to a cooperative change in the chain network in a light-responsive polymer gel, ultimately presenting macroscopic motion. A few examples of light-responsive polymer gels are discussed below, where the specific mechanical motion of photo-active molecules is exploited to achieve macroscopic motion.

Amplification of molecular switching was achieved successfully in polymer gels via noncovalent interactions. Naumov et al. have designed and synthesized multi-responsive films that not only change their emission color under $\mathrm{pH}$ variation (acidochromism) but also display macroscopic motion in response to humidity and light. ${ }^{51}$ The polymer gel used by Naumov et al. was formed by blending an alkene derivative BHSB as a light-active element and an agarose gel matrix, the hygroscopicity of which is known to be high, which facilitates the humidity-driven response of this hybrid gel (Figure 2.4). In additional to its acidochromism and humidity gradient-induced deformation, the hybrid film exhibited 
Strategies to amplify the light-driven motion of molecules up to the macroscopic level

various deformations under exposure to UV light, in its dry state (Figure 2.4b). The dimensions of the film strip, including its aspect ratio, were shown to influence the macroscopic mechanics, and the film strips were either spiral, twisted or bent, which suggests that the motion of each strip is likely dependent on the orientation of the guest molecule BHSB in the film. For example, a strip with a relatively long length and a narrower width bends under UV light (Figure 2.4b (iii)). Upon bending, a plot of the position of the apex of the film as a function of time highlighted four mechanistic steps (Figure 2.4b (iv)), involving the conversion of light into kinetic energy, potential energy, and the relaxation of the film. Surprisingly, this transformation of light into motion was successfully performed by trans-cis switching of the alkenes, even though the BHSB containing these photo-active groups was included in the materials through non-covalent interactions (Figure 2.4c). Overall, the system was found to lack certain directionality in yielding a target motion, probably due to the lack of control over molecular orientation.

a

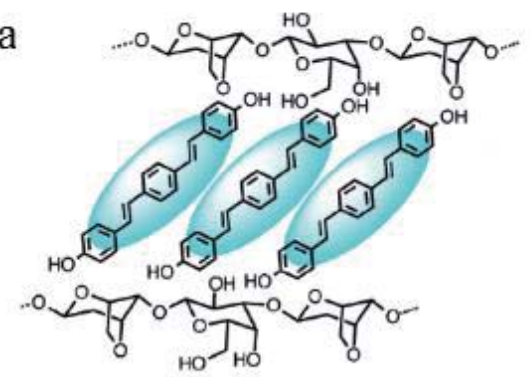

$\mathrm{c}$

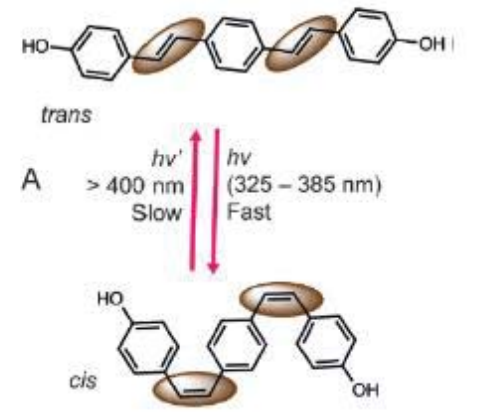

$\mathrm{b}$

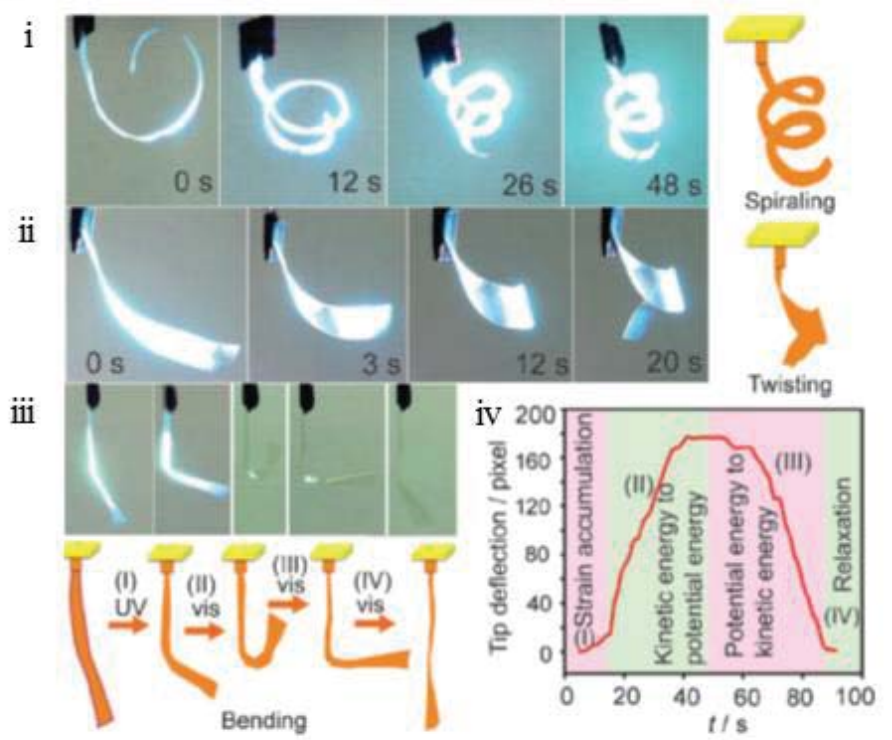

Figure 2.4 a) Structure of agarose-based hybrid film, non-covalently bearing 1,4-bis(parahydroxystyryl)benzene (BHSB). b) Mechanical response of hybrid strip upon light exposure. i) UV-triggered spiraling; ii) UV-triggered twisting; and iii) sequence of UV and visible light-triggered bending (UV exposure in first two panels and visible light exposure in last three panels). iv) Bending site of film tips over time, showing four phases of energy conversion: c) trans-cis isomerization of BHSB. Adapted with permission from [51] (Copyright 2015 WILEY-VCH Verlag GmbH \& Co. KGaA, Weinheim). 
The unidirectional rotary motion of molecular motors has also been harnessed in lightresponsive polymer gels. A second-generation motor with high molecular speed, ${ }^{52}$ was functionalized by PEG linkers terminated by an alkyne at the lower half, and an azide at the upper half, respectively (Figure 2.5a). The modified motor was transformed into a lightactive polymer gel via a click reaction. Interestingly, gel 9 displays large macroscopic shrinkage under irradiation with light in toluene, with up to $\sim 80 \%$ volume change (Figure 2.5b). The original size of gel 9 was restored with longer irradiation times (Figure 2.5b, (iii)(iv)).

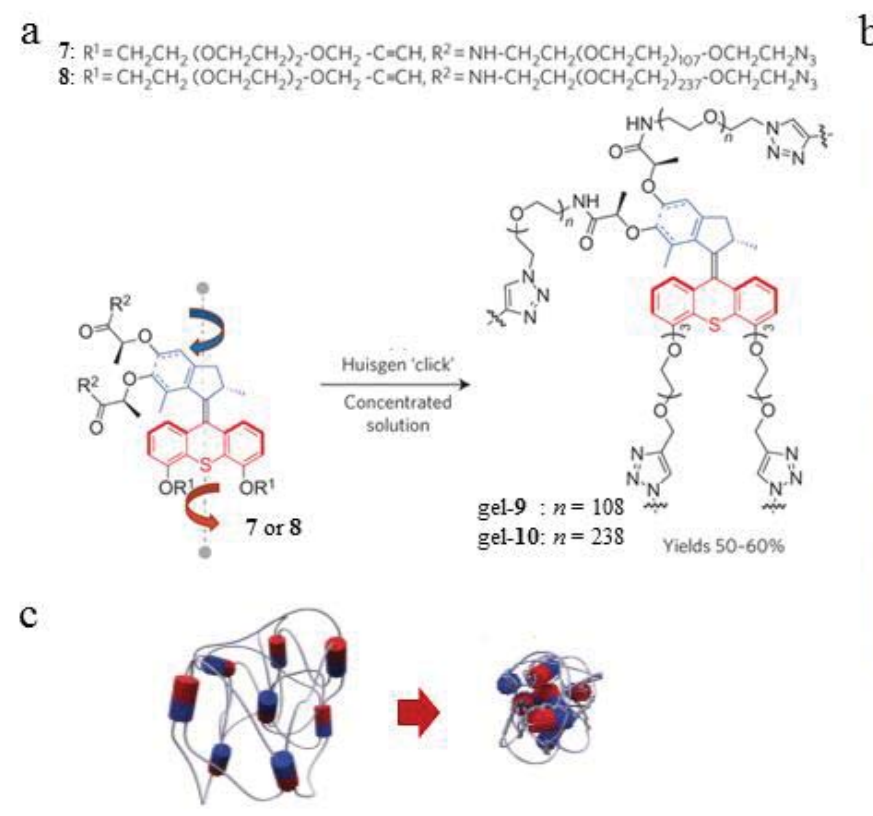

b
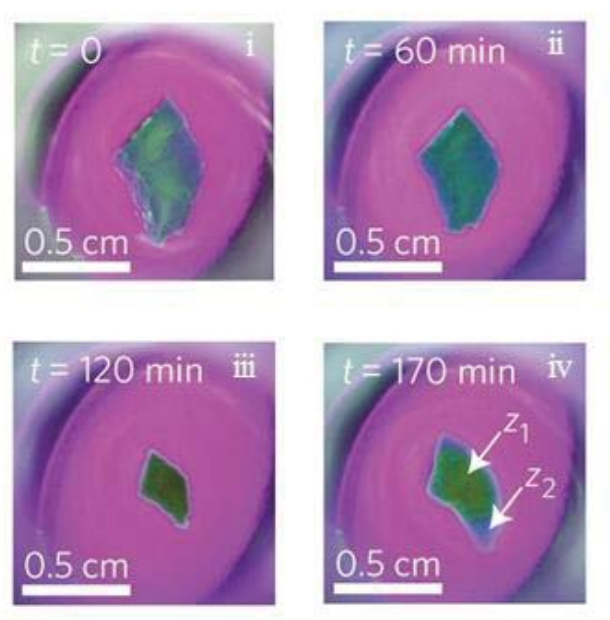

Figure 2.5 a) Design of motor monomers and click reaction to form chemical gel $\mathbf{9}$ and $\mathbf{1 0}$. b) Photo-induced macroscopic contraction of gel $\mathbf{9}$ in toluene ( $\mathrm{z}_{1}$ indicates a region that is still contracted and $z_{2}$ indicates a region that has started to disrupt). c) Proposed model presenting unidirectional rotatory mechanics of a motor in polymer gels. Adapted with permission from [52] (Copyright 2015 Nature Publishing Group).

In contrast, a gel made with an analogous monomer, but with the alkene moiety replaced with an episulfide group, showed no response to light. The inactivity of the control gel bearing the episulfide moitey and the observation of larger pore sizes in gel $\mathbf{9}$ under light irradiation, allow one to conclude that the overcrowded alkene performs as a rotary motor unit in the active gel $\mathbf{9}$, by twisting up towards a more entangled state under irradiation with light (Figure 2.5c). This rotation leads to a tighter network, which then affords a larger space 
Strategies to amplify the light-driven motion of molecules up to the macroscopic level

in between wound polymer (larger pores), and finally results in macroscopic shrinkage of the gel. Interestingly, the gel shrinkage can also be considered as an energy storage system, in which the motor unit transforms light energy and stores it in the form of a twisted gel network.

While polymer gels have proved efficient in delivering macroscopic deformation and motion with large amplitudes, these soft structures also require wet working conditions and their structure is usually not very well defined, ${ }^{55}$ which makes their motion less predictable ${ }^{51}$ and thus less relevant in terms of practical applications. A strategy to address these limitations, uses matrices that display both elasticity and highly ordered structures. ${ }^{56}$

\subsubsection{Light-responsive liquid crystal polymer networks}

Incorporating photo-responsive molecular switches into liquid crystal polymer networks is an efficient strategy to amplify molecular motion, leading to programmable and adaptive mechanics. ${ }^{57}$ In contrast to polymer gels, liquid crystal polymer networks combine both the elastic properties of a polymer and the orientational order of liquid crystals. ${ }^{58}$ The orientational order of liquid crystal polymers manifests an anisotropy that promotes creation of macro-scale motion aside from the assist of its polymer-like character. The resident photoactive molecule is triggered into motion by light, and the anisotropy of the liquid crystal polymer would then allow the liquid crystal network to cooperatively re-orient in a systematic manner, namely with a preferred direction. As a result, the motion of each unit in the liquid crystal polymer would be uniformly integrated. ${ }^{55}$ This is capable of producing high amplitude and expectable macroscopic motion based on the well-defined orientational structure.

By comparison, polymer gels are amorphous materials, which essentially means that their structure is random and lacks any anisotropic features. When molecular motion is triggered by light, the gel network adjusts into another conformation, but in a disordered fashion. Due to the unfavorable directionality, the relative motion of each polymer gel unit is then offset, leading to unpredictable and low degree of macroscopic motion. Accordingly, a liquid crystal polymer is superior to a polymer gel in terms of motion control, both of amplitude and directionality. In addition, liquid crystals display high responsiveness and reversible features. The high responsiveness of a liquid crystal mesogen to an external trigger confers higher 
sensitivity on the liquid crystal polymer than a polymer gel. This means that the motion of a photo-active molecule in a trivial site of the network is sufficient to create macroscopic motion in the bulk liquid crystal polymer. Moreover, the ability to re-orient a liquid crystal mesogen also facilitates reversibility in the performance of liquid crystal polymer materials.

a Liquid crystal main-chain polymers

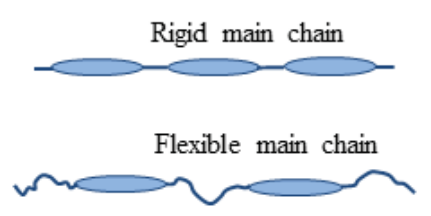

b

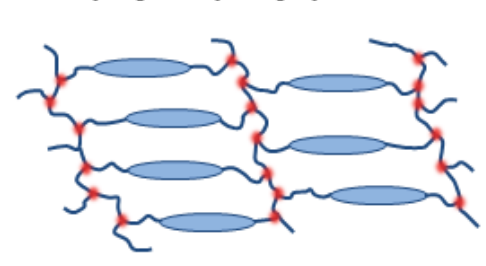

c Liquid crystal elastomers

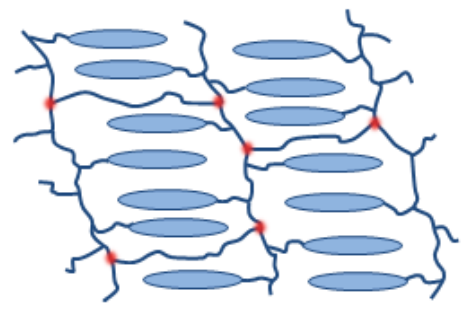

Figure 2.6 Representation of distinct structure of: a) liquid crystal polymers (LCPs); b) glassy liquid crystal polymer networks (LCNs); and c) liquid crystal elastomers (LCEs). Adapted from [57].

Historically, there are family terms for polymer materials incorporating liquid crystal for instance liquid crystal polymers (LCPs), polymeric LCs, liquid crystal elastomers (LCEs) and liquid crystal polymer networks (LCNs). They are defined based on the classification of their properties, such as cross-link density and thermomechanical properties. ${ }^{57}$ Here, we refer primarily to liquid crystal elastomers and liquid crystal networks (see Figure 2.6). Even though both of these materials are composed of a cross-linked liquid crystal, they can be distinguished by their network density. Liquid crystal polymer networks are characterized by their moderate-to-densely crosslinked network, while liquid crystal elastomers are loosely crosslinked networks that are associated with high flexibilities as well as large deformations in respond to stimuli. The flexible backbone of liquid crystal elastomers is often formed by a polysiloxane. Finkelman et al. were the first to invent polysiloxane-based liquid crystal elastomers in which a photo-switch was embedded, which permitted them to contract/ extend by up to $20 \%$ in response to light irradiation. ${ }^{59}$ Building on this, research groups lead by Ikeda ${ }^{60}$ and Broer ${ }^{61}$ have engineered glassy liquid crystal polymer networks that also display photo-induced macroscopic bending.

Designing the appropriate architecture for the liquid crystal polymer is key to achieving large macroscopic motion. The orientation of the liquid crystal, its chemical composition, the incorporation of the photo-active molecules, and the fabrication method are all parameters 
Strategies to amplify the light-driven motion of molecules up to the macroscopic level

that determine the final architecture of the liquid crystal polymer and thus define its mechanical motion and deformation at the macro-scale. In addition, using light as an external trigger allows additional control parameters, including polarization and the exposure area (bulk or localized area). These approaches have typically resulted in two modes of macroscopic motion from different liquid crystal polymers, including bending and torsional modes.

Below we provide a brief review of the designs of liquid crystal polymers that display intriguing motion, including bending and torsional motion, based on the amplification of nanoscale motion. Because control of the molecular orientation is key to optimizing the performance of these materials, some classical examples of liquid crystal orientation are introduced first, with a brief introduction to the commonly accepted mechanisms yielding photo-induced macroscopic motion.

\subsubsection{Macroscopic organization of liquid crystal polymers in thin films}

Control of the liquid crystal orientation is a pre-requisite to gain control over the macroscale deformation of liquid crystal polymers. Liquid crystals are particularly sensitive to boundary conditions, meaning that their orientation can be promoted by subjecting the interfaces to specific treatments. Usually, a polymer-coated glass slide is used for that effect. $^{62}$ The induced alignment of the liquid crystal stems from the hydrophobic intermolecular interaction of the liquid crystal molecule with surface anchoring, for example via Van der Waals forces, dipole-dipole interactions, or hydrogen bonding. A variety of alignment techniques can be used to prepare surface anchoring, depending on the target material and its intended application, e.g. rubbing, evaporation, and photo-alignment. Complex orientation patterns are relatively easy to achieve using the photo-alignment technique. ${ }^{63}$ Typical organizations of liquid crystals that can be promoted in thin films by controlling the orientation at the interfaces are shown in Figure 2.7, including uniaxial planar, homeotropic, splay, and twist organizations. Planar anchoring at both interfaces promotes inplane alignment of the director, with the liquid crystal molecules preferentially oriented parallel to the substrate (Figure 2.7a), while homeotropic (perpendicular) anchoring is used to induce preferential alignment perpendicular to the plane (Figure 2.7b). In twist and splay 
liquid crystal ordering, the liquid crystal director is subjected to a gradient in orientation, yielding a $90^{\circ}$ rotation in the plane of the substrate (Figure 2.7c) and a $90^{\circ}$ rotation perpendicular to the plane of the substrate (Figure 2.7d), respectively.
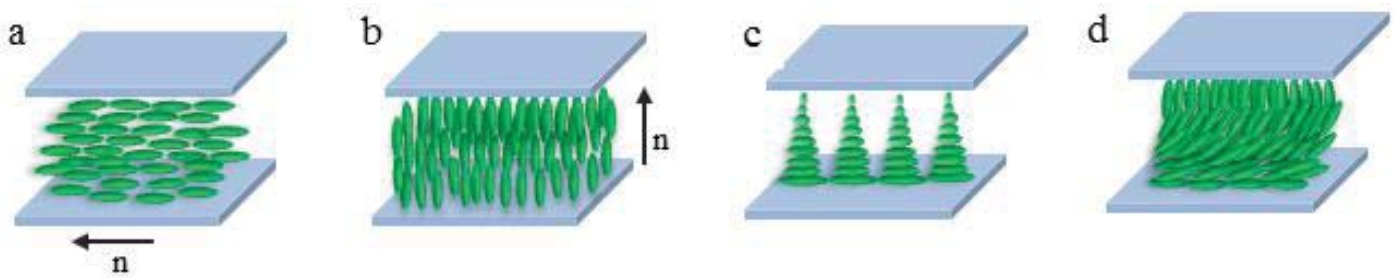

Figure 2.7 Director profiles in thin films of liquid crystals, promoted by boundary conditions at the interface: a) planar uniaxial; b) homeotropic; c) twist; and d) splay orientations.; the director (n) represents the average local orientation of the liquid crystal.

\subsubsection{Photo-actuation mechanism in liquid crystal polymer networks}

In liquid crystal polymer networks incorporating photo-switches, it is generally accepted that the photo-actuation mechanism stems from the disorder introduced upon molecular switching. ${ }^{64-66}$ A few molecular switching events can disrupt the long-range orientational order of liquid crystals, resulting in a phase transition from the ordered nematic phase to the disordered isotropic phase (Figure 2.8). The reduction in order is accompanied by the development of a contractile strain, which causes an anisotropic contraction along the molecular director, and a perpendicular expansion. Azobenzenes are the most commonly used photo-switches because of the structural changes that occur during trans-cis photoswitching: the rod-like geometry of the trans-isomer is compatible with the orientational order of the liquid crystals, whereas the bent shape of the cis-form is incompatible, which generates disorder (Figure 2.8). This process is reversible as the liquid crystal re-orients, either under the effect of the back-isomerization once irradiation has stopped, or by optically induced back-isomerization of the azobenzenes. Interestingly, it has been demonstrated that photo-actuation of liquid crystal polymer networks by order loss of liquid crystal during light irradiation is also accompanied with a free volume increase/ density decrease of the films. ${ }^{67}$ 


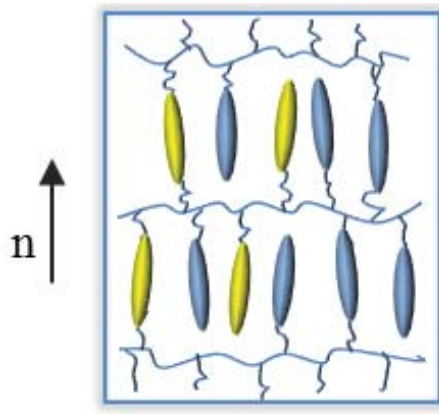

$\mathrm{N}$ phase

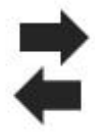

I phase

Figure 2.8 Change of mesogenic orientational order by manipulation of resident photo-active molecules and the resulting macroscopic mechanical motion. Adapted from [64 and 68].

\subsubsection{Bending modes}

In relatively thick films or with high absorption of photo-switchable units, liquid crystal polymer films display a bending motion. The mechanism of bending mostly relies on unequal contraction through the depth of the films under non-uniform illumination. ${ }^{7,69}$ Such unequal contraction originally arises from attenuation of light through the film depth, which induces molecular motion and corresponding strain, being more pronounced on the side where light enters. Such a strain gradient across the depth of the film forces the film to accommodate imbalanced contraction over its depth, and eventually it bends. Specific liquid crystal ordering yields distinct bending behaviors in particular, in terms of bending direction and amplitude.

The light-induced bending of liquid crystal polymer networks was reported by Kondo et al. (Figure 2.9a), ${ }^{70}$ who observed thin film with either uniaxial planar or homeotropic orientation. Interestingly, both films were found to bend under light irradiation, yet in opposite directions. The planar film bends towards the light source while the homeotropic film bends away from it (Figure 2.9b). This difference in bending direction originates in the coupling of a gradient in trans-cis photo-isomerization with the specific orientation of the material, as depicted in Figure 2.9c. In both cases, due to the high absorptivity of azobenzenebased monomer at the working wavelength of light, the light intensity is attenuated through the film thickness. Therefore, the upper surface of the film, being closer to the light source, experiences greater deformation, which causes shrinkage along the molecular director and 
perpendicular expansion. Accordingly, the upper side of the planar film contracts along the plane of the film because the director is aligned horizontally, and bending occurs toward the light source (Figure 2.9c, (i)). Conversely, the upper side of the homeotropic film expands in-plane because the director is aligned vertically, which results in the film bending away from the light (Figure 2.9c, (ii)). These results indicate that the direction of bending can be encoded in the structure of liquid crystal polymers, via orientational control.

a
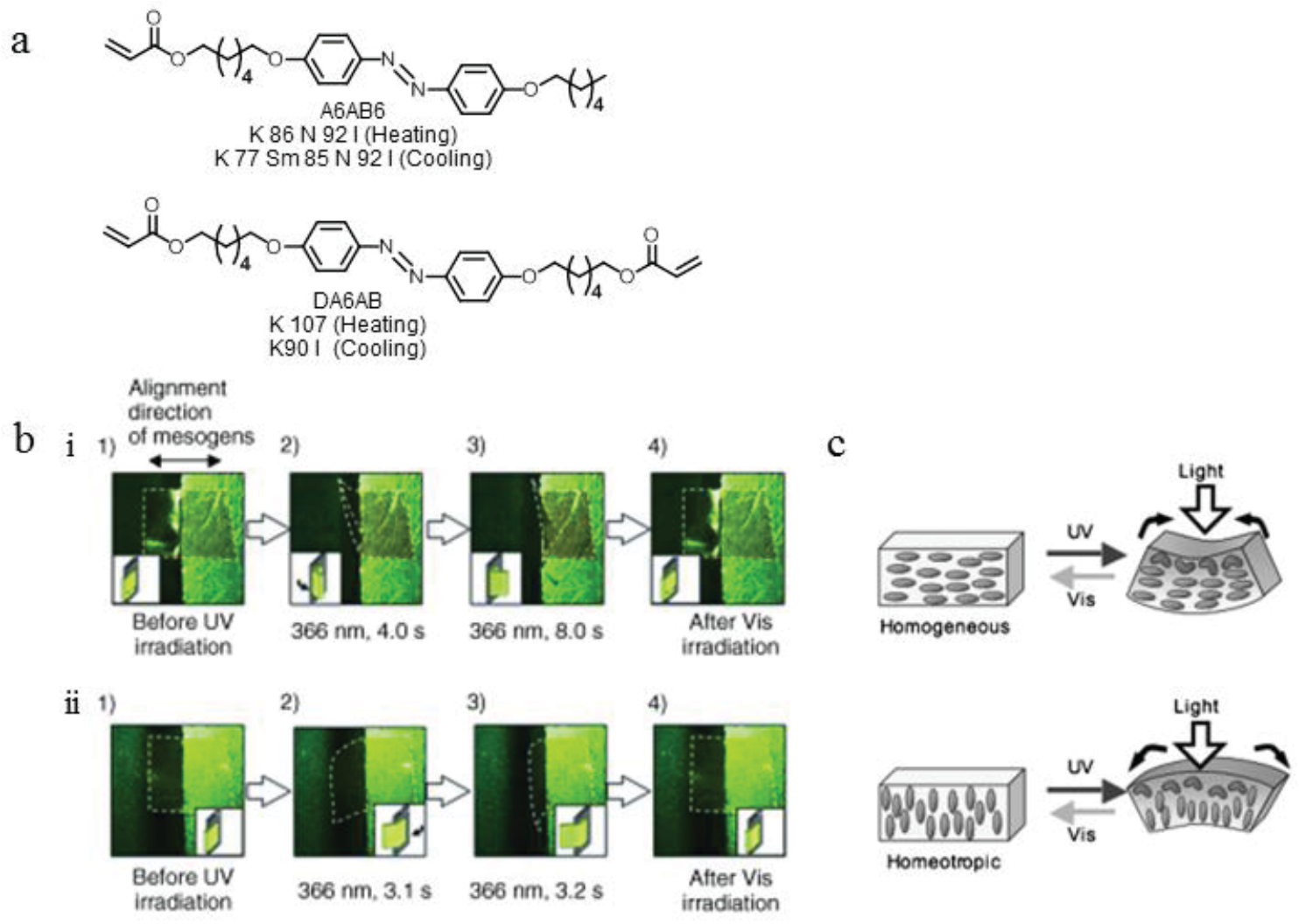

Figure 2.9 a) Structure of liquid crystal mesogen A6AB6 and cross-linker DA6AB. b) Photoresponsive bending and reversibility of films with uniaxial planar (i) and homeotropic orientation (ii). c) Bending mechanism dictated by uniaxial planar and homeotropic ordering. Adapted with permission from [70] (Copyright 2006 WILEY-VCH Verlag GmbH \& Co. KGaA, Weinheim).

The molecular orientation has also been shown to affect the degree of bending. With the aim of enhancing the bending amplitude, as required for micromechanical devices, van Oosten et al. have developed glassy liquid crystal polymer films with a variety of director profiles, including uniaxial planar, twist and splay orientation, and compared their bending amplitudes upon activation with light. ${ }^{71}$ The experimental results were in agreement with 
Strategies to amplify the light-driven motion of molecules up to the macroscopic level

simple models, in that bending for twisted and splay orderings has a greater amplitude than the bending observed in films with uniaxial planar orientation, under identical irradiation conditions. The model involving strain development of a film after triggering with light suggests that a film with uniaxial orientation shows only contractile behavior across the thickness of film. By placing the side with horizontal alignment upwards (towards the light source), the twisted and splayed films contracted in the upper layer of the films and expanded in the lower layer, resulting in a greater strain gradient across the thickness of the films than that generated in a uniaxial planar film, and consequently affording greater bending amplitude.

Engineering the bending function can also be achieved by manipulating the properties of the light chosen to activate molecular motion. ${ }^{60,72-74} \mathrm{Yu}$ et al. examined a polydomain liquid crystal polymer film, which was found able to tune different bending modes by using different polarization directions of linearly polarized light. ${ }^{60}$ The molecular orientation of such polydomain films, bearing an azobenzene moiety as the photo-active unit, is composed of several micro-domains. In each domain, the molecular director oriented uniformly, i.e. in the same direction, but oriented differently between neighboring domains (Figure 2.10b). ${ }^{55}$ Exploiting the selective absorption of azobenzene units among micro-domains as dictated by their distinct orientation, only those domains where the azo-benzene unit oriented in the identical direction with the polarized light would contract, so giving rise to bending of the films in a direction in line with the polarization direction (Figure 2.10a). Based on this selectivity, a cycle of film bending and recovery with a variety of bending modes could be controlled by irradiation with a series of polarization directions $\left(0^{\circ},-45^{\circ},-90^{\circ}\right.$ or $\left.-135^{\circ}\right)$ of linearly polarized light, each of which was followed by visible light irradiation to convert the film to its original state (via cis-trans isomerization of the azobenzene unit). 
a

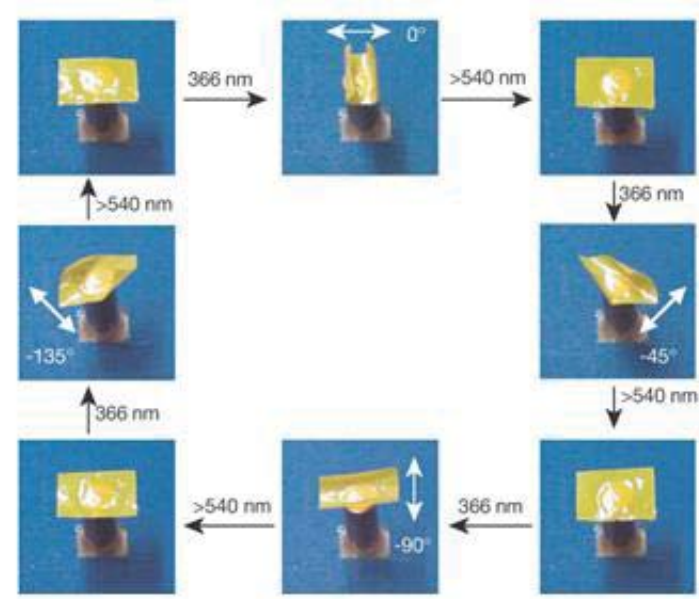

$\mathrm{b}$

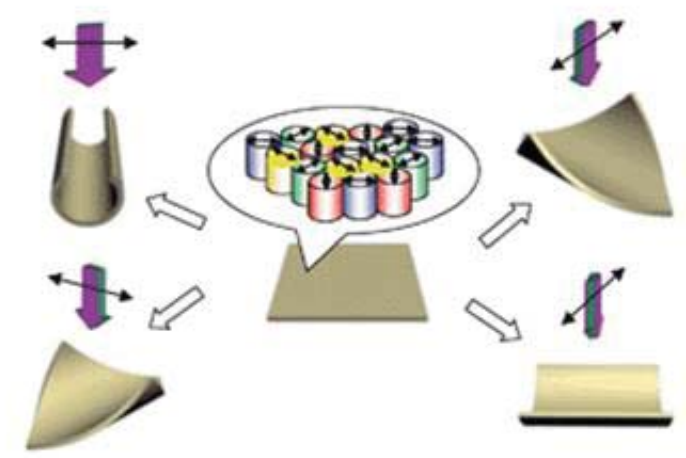

Figure 2.10 Four distinct bending states of liquid crystal films under the control of four polarization directions of linearly polarized light $\left(0^{\circ},-45^{\circ},-90^{\circ}\right.$ or $\left.-135^{\circ}\right)$, with intervening subsequent recovery of the flat state by irradiation with visible light. Adapted with permission from [60 and 55] (Copyright 2003 Nature Publishing Group and 2012 Royal Society of Chemistry).

Another example of controlling the light condition to induce photo-mechanical bending was reported by White, et. al. Using linearly polarized light with a particular wavelength, intensity, and exposure area, a cantilever of monodomain liquid crystal polymer containing an azo-switch molecule displayed oscillation of bending with high amplitude (up to $170^{\circ}$ ) and frequency, resembling the flapping speed of a hummingbird's wing (Figure 2.11a). ${ }^{72}$ Unlike the common mechanism of trans-cis azobenzene isomerization driven by UV light, the strategy here exploited linearly polarized light in a wavelength range that both trans- and cis- isomers absorbed almost equally $(440-515 \mathrm{~nm})$, which in turn caused transcis-trans re-orientation (Figure 2.11b). Similarly, this molecular re-orientation of the azobenzene unit resulted in a macroscopic change of the network by contraction along the direction of polarization and expansion in the perpendicular direction, eventually inducing bending. To acquire the oscillating mode of bending, the light exposure was applied locally to the base of the film cantilever. Due to the dependency of the bending angle on film thickness, the light intensity was also adjusted so that when the first bent state reached its full angular limit, it led to concealment of the just-irradiated side of the film (front side), allowing exposure of the opposite side of the film (back side) (Figure 2.11b, (iii)). These processes caused the reverse direction of bending, which in the end led to alternating re-exposure to light on each side of the film, thereby creating a cycle of up-down bending. Briefly, with 
Strategies to amplify the light-driven motion of molecules up to the macroscopic level

particular light conditions, this oscillation was forced by contraction of the film on the irradiated side owing to molecular reorientation accompanied by recovery of the network during shielding from the light.
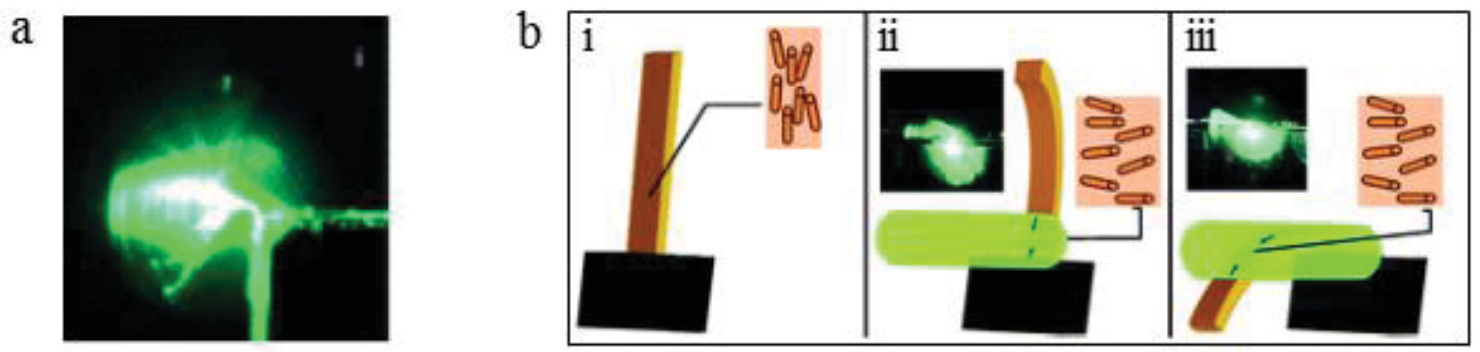

Figure 2.11 a) Oscillation of film under control of linearly polarized laser light, the direction of polarization being parallel to the molecular director. b) Model mechanism of molecular re-orientation of liquid crystal cantilever under control of light, promoting macroscopic bending with oscillation. Adapted with permission from [72] (Copyright 2008 Royal Society of Chemistry).

The bending function can be controlled by another factor than molecular orientation and the projection of light, viz., the geometrical shape of the liquid crystal polymer.

Taikai et al. have recently demonstrated that liquid crystal polymers shaped as a fiber can bend in three dimensions depending on the pathway of light irradiation (Figure 2.12), which shows great promise for the development of artificial muscles. ${ }^{75}$ In contrast to a flat film fabricated in confining glass plates, this fiber-like material containing an azo-functional switch was produced in two steps: copolymerization of photo-active liquid crystal monomers followed by fiber formation by picking the fiber with a toothpick during copolymerization of the active-copolymer and cross-linker. Inspection under the polarized optical microscope revealed that the fiber possessed well-aligned molecular organization, showing a clear contrast at each $45^{\circ}$ turn of the fiber relative to the analyzer. Using a trans-cis switching mechanism similar to that of the photo-induced bending of a flat film, the fiber bent towards the light as the side of the fiber that is exposed to the light contracts more strongly. In contrast to the dimensional bending of a flat film, this fiber can bend in multiple directions based on the approaching pathways of the light. The bending ability in all three dimensions was dictated by the cylindrical geometry of the fiber, which allowed access to the light and a corresponding contraction of the film in any direction. 

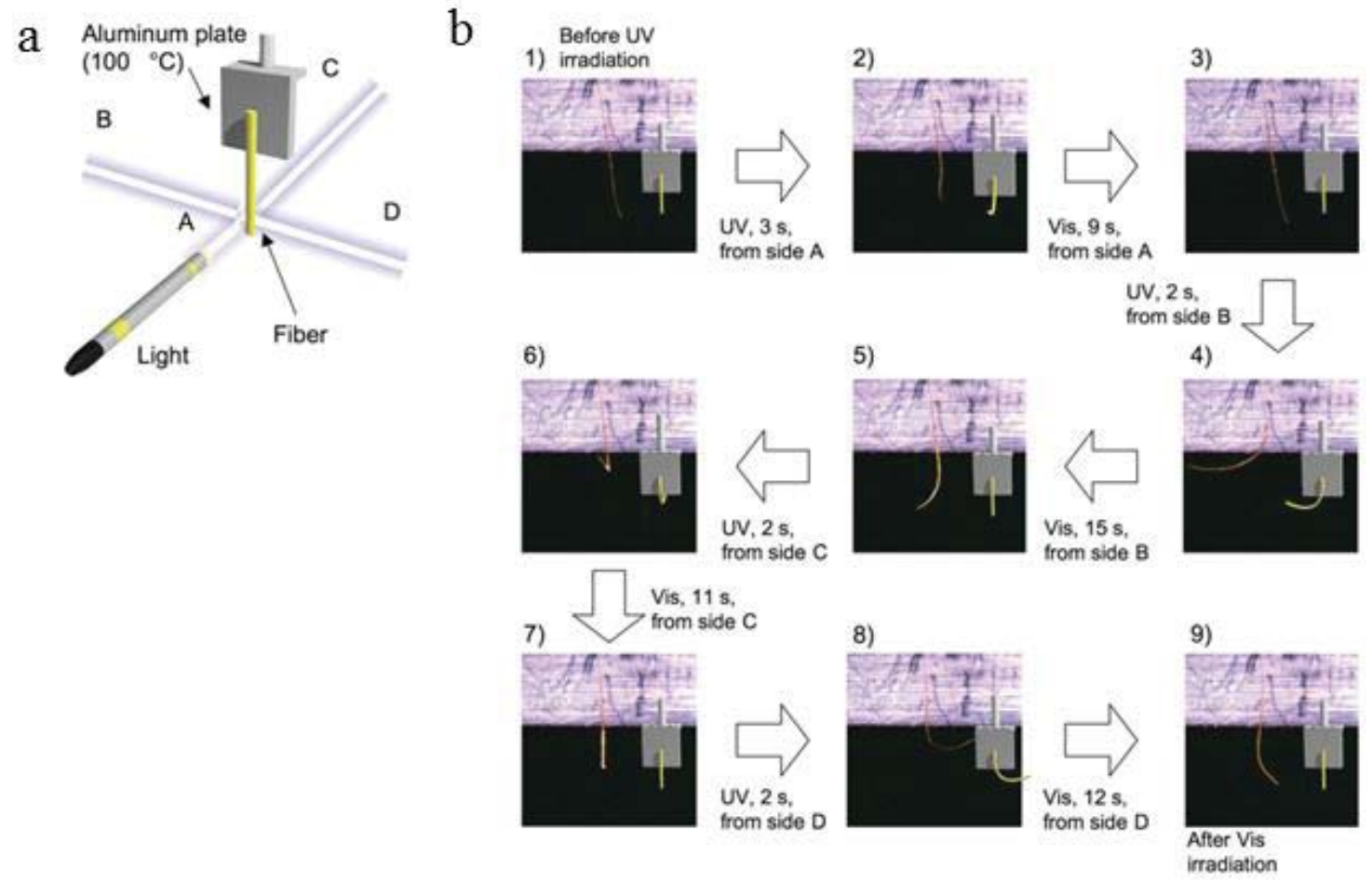

Figure 2.12 a) Diagram of photo-response study showing distinct paths of light (A, B, C and D). b) Bending behavior of fiber after triggering with UV light and subsequent recovery of the film under control of four different light paths. Adapted with permission from [75] (Copyright 2010 WILEY-VCH Verlag GmbH \& Co. KGaA, Weinheim).

To gain additional functionality in the use of liquid crystal polymer bending, a strategic approach consists in using complementary materials with liquid crystal polymer films. ${ }^{76,77}$

Targeting biological applications, the integration of liquid crystal polymer bearing an azotolane-moiety and upconversion material was achieved by Jiang et al. to eliminate the barrier to high energy UV light in common liquid crystal polymer films. ${ }^{77}$ The upconversion materials are polyurethane-based films, consisting of dual dyes that are capable of absorbing longer wavelength red light (by dye-sensitizer) and emitting the shorter wavelength blue light (by dye-emitter). Such blue emissions are matched to the light needed to trigger isomerization of azotolane. Pasting this upconversion film on top of the liquid crystal polymer containing an azotolane moiety, either by adhesive or direct spin coating, generates a composite material that can bend in response to red light based on emission-reabsorption between the films. The use of red light with low intensity required for such a composite film opened the way to biological applications due to effective penetration into tissue and being benign to cell of the red light than light in shorter wavelengths. 
Strategies to amplify the light-driven motion of molecules up to the macroscopic level

Alternatively, macroscopic bending of soft materials has been achieved by embedding molecular switches in liquid crystal polymer networks. Azobenzene switches were used primarily, ${ }^{5}$ but beyond azobenzenes, Mamiya et $a .^{78}$ recently reported the use of diarylethenes as alternative photochromic switches for liquid crystal polymer networks. Specifically, the photoactive unit that they designed was composed by a liquid crystal unit, connected to a diarylethene core (Figure 2.13a). Under irradiation with UV light, the liquid crystal polymer networks incorporating that photo-responsive unit demonstrated bending. Noticeably, the amplitude of bending was small, likely because the disorder induced by shape modification of a diarylethene is much more moderate than when created by the trans-to-cis isomerization of an azobenzene (Figure 2.13a and b, (i)-(ii)). Intriguingly, once bent, the liquid crystal elastomer exhibited thermal stability, i.e. it did not return to its initial shape, even upon heating (Figure 2.13c). However, it reverted to the initial flat state when visible light was used to induce the reversion (Figure 2.13c, (ii)-(iv)). This macroscopic behavior has to be compared to the photochemistry of the diarylethene switch itself, that displays thermal stability in both closed and open forms and can only isomerize between both forms by using irradiation with light (Figure 2.13a). Unlike the temperature-induced reversibility that is typical of liquid crystal elastomers incorporating azobenzenes, thermal stability of this film was thus achieved by harnessing the specific molecular properties of the diarylethene switch. 
a

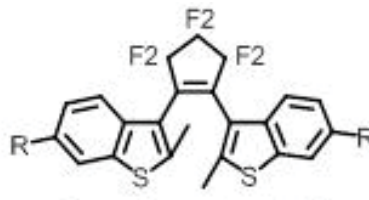<smiles>[R]CC(=O)Oc1ccc(-c2ccc(O[14CH2]OC(=O)C=C)cc2)cc1</smiles>

Open form (6-o, 7-o)

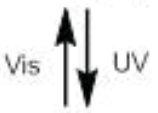<smiles></smiles><smiles>[R7]CC1=C2c3ccc([R])cc3SC2(C)Sc2cc([R])ccc21</smiles>

Closed form (6-c, 7-c)

b
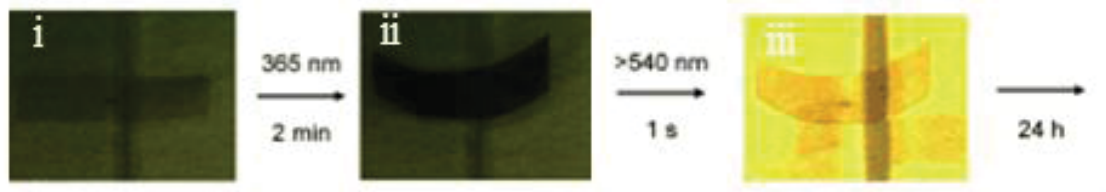

iv c

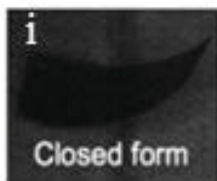

ii

$120^{\circ} \mathrm{C}, 36 \mathrm{~h}$

Closed form

Figure 2.13 a) A liquid crystal incorporating a diarylethene photo-switch. b) UV-driven bending and reverse motion triggered by a combination of irradiation with visible light and thermal recovery at room temperature. c) After irradiation with UV light, the film does not recover its initial shape upon simple heating. Adapted with permission from [78] (Copyright 2015 WILEY-VCH Verlag GmbH \& Co. KGaA, Weinheim).

\subsubsection{Torsional modes}

A liquid crystal polymer can also display torsional motion by magnifying nano-scale motion. The torsional mode is observed when the molecular director is aligned in particular directions, often offset from the main axis of a rectangular strip of liquid crystal polymer film. ${ }^{79,80}$ Similar to the bending mode, non-uniform contraction occurred between the sides of the film strip due to gradient absorption of light by illumination, though it is combined with offset strain contraction caused by offset alignment of the molecular director relative to the main axis. These effects thereby dictated that the film adopts a torsional motion. As strain generation was directed by the molecular motion of photo-active molecules and cooperativity 
Strategies to amplify the light-driven motion of molecules up to the macroscopic level

of the bulk liquid crystal mesogen, the directionality and amplitude of macroscopic torsion of the films were then correlated to the inclination of molecular alignment in respect to the main axis of the strip, refered to as the offset angle.

Harris et al. observed that a cut strip of a film of azo-containing liquid crystal polymer with a twist configuration, where the molecular director on each face of the strip has an offset angle of $45^{\circ}$, exhibited coiling motion under illumination with UV light. ${ }^{81}$ The trans-cis switching of the azo-unit as well as the inclined molecular alingnment scaled to the main axis of the strip accounted for this coiling deformation.

Moreover, trans-cis-trans re-orientation was also utilized as mechanical motion to trigger macroscopic torsion of films. Such a strategy could be achieved by choosing light in the visible range, where absorption is equal between the trans and cis forms. Using linearly polarized visible light $(442 \mathrm{~nm})$, Lee et al. examined the behavior of monodomain azocontaining cantilevers with different imposed molecular alignments (Figure 2.14). ${ }^{82}$ In contrast to the bending mode, where the molecular directors were imposed along the cantilever axis, the twist modes were obtained from cantilevers with offset alignment relative to the main axis. In such systems, the maximum torsion was presented at an offset angle of $65^{\circ}$. Torsional magnitude was found to depend on the degree of inclination of the molecular alignment. Meanwhile, the directionality of torsion (either clockwise or anti-clockwise twist) was defined by the direction of the alignment as exemplified by the opposite twist of cantelivers with offset angle $-65^{\circ}$ and $65^{\circ}$ (Figure $2.14 \mathrm{~b}$ ). In a further investigation, ${ }^{83}$ a flatshaped cantilever with offset molecular alignment, prepared from films with twist and hybrid molecular orientation, was also demonstrated to undergo a higher degree of torsion, which is ascribed to complementary contracting/expanding of distinct offset alignment between both faces of either twist or hybrid geometry films.

Instead of using offset angle of molecular alignment, torsional motion was also observed by adjusting the polarization direction of light to intermediate angles with respect to the strip's axis. Tabiryan et al. investigated light-responsive mono-domain strips where the molecular director aligns along the strip's long axis, upon irradiation with linearly polarized light with $45^{\circ} /-45^{\circ}$ polarization relative to the long axis of the strip (Figure 2.15). ${ }^{84}$ In contrast to the use of direction of polarization along either the short or long axis of the strip to cause bending, a slight twisting of the strips was observed by tilting the direction of polarization to 
those intermediate angles. Interestingly, the twist direction was inverted by inversion of the polarization angle (Figure 2.15b and c).
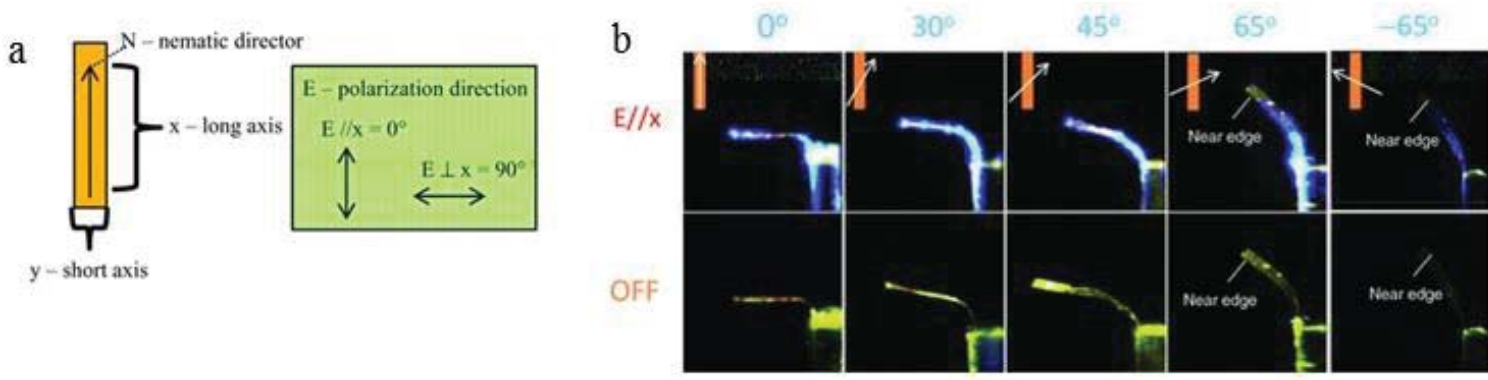

Figure 2.14 a) Representation of alignment director on cantilever dimension and direction of light polarization; b) in-plane bending $\left(0^{\circ}\right)$ and out-of-plane twisting of cantilevers with offset angle $\left(30^{\circ}, 45^{\circ}, 65^{\circ}\right.$ and $\left.-65^{\circ}\right)$. Adapted with permission from [82] (Copyright 2013 Royal Society of Chemistry).
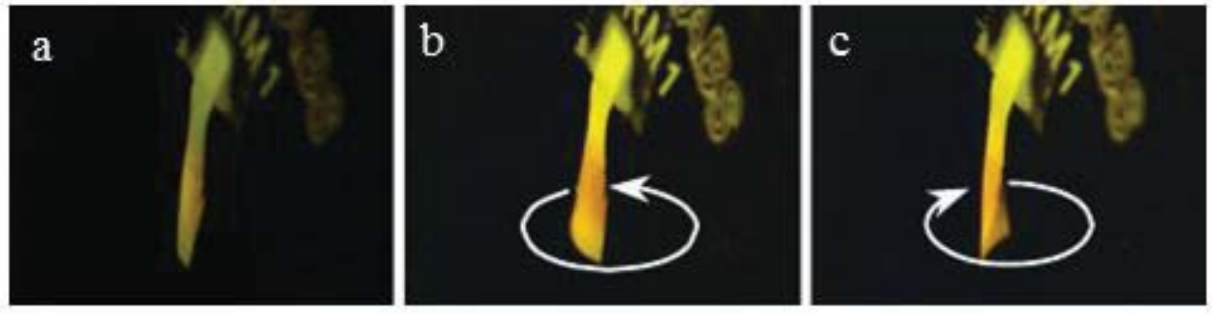

Figure 2.15 a) Strip film before light illumination. b) and c) Twisting of strip films with counter-clockwise and clockwise turn under $-45^{\circ}$ and $45^{\circ}$ polarization relative to long axis of strip, respectively. Adapted with permission from [84] (Copyright 2005 Optical Society of America).

\subsection{Macroscopic motion at work}

Translating motion from the molecular level up to the macroscopic scale is fundamentally relevant, but it also holds promise in terms of applications that can provide useful work at the macroscopic level. The smart design of rotating plastic motor, ${ }^{85}$ micro-pump, ${ }^{61}$ microvalve, ${ }^{86,87}$ and micro-robot arms ${ }^{88}$ have demonstrated the promise of this approach.

Utilizing the translation of molecular mechanics into macroscopic dimensional change, Schiphorst et al. developed a light-responsive polymer gel bearing spiropyran derivatives as an adaptive valve in a microfluidic system. ${ }^{87}$ Profiting from the hydrophilicity and hydrophobicity of the open and closed forms of spiropyran under light-driven conditions, macroscopic swelling and shrinkage of the gel under the action of water (uptake/ release) 
occur reversibly, enabling the gel to function as a size-tunable valve in a channel (Figure 2.16). Using a new design of spiropyran and a self-protonating gel formulation, this gel valve approaches the practical limit of valve closing, while suffering from slow re-swelling and the need for acidic working conditions. Briefly, this application showed that mechanics at the molecular scale under light manipulation compelled the valve to do a useful task at the macro scale, namely opening to let fluid flow or closing to block it.

a

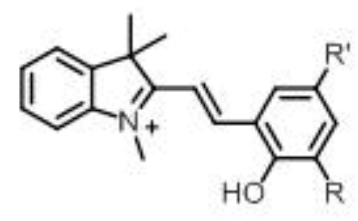

b

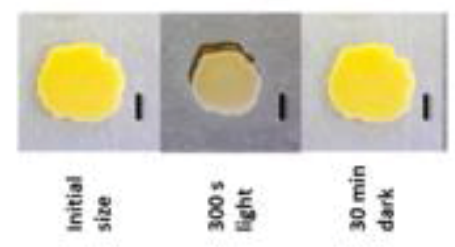

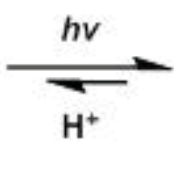

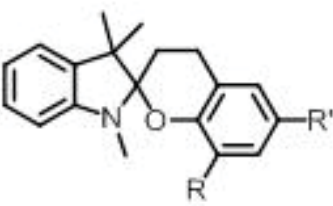

C

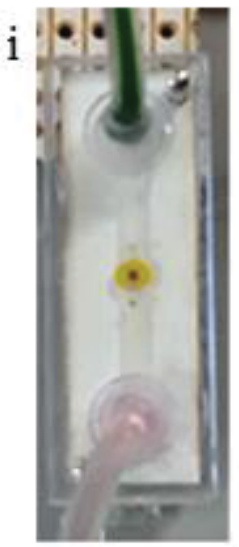

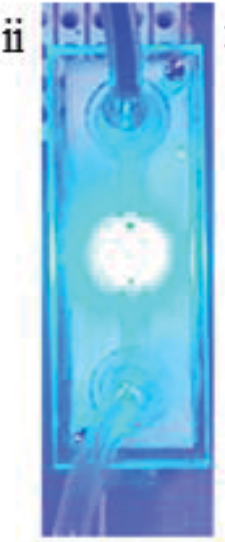

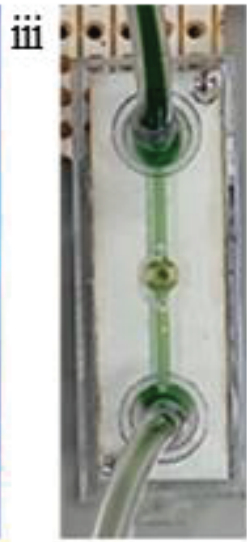

Figure 2.16 a) Light-induced switching of spiropyrans. b) Dimensionality of free-standing gel in initial, irradiated, and reversed states, respectively. c) Operation of gel valve: i) closure of channel by swelling gel (no liquid flow); ii) local irradiation with blue light; iii) opening of channel by shrinking gel valve, occurring after 1 min of irradiation (green liquid flow). Adapted with permission from [87] (Copyright 2015 American Chemical Society).

Liquid crystal polymers have also demonstrated the potential to do work. Going beyond common macroscopic bending, which limits real applications, Ikeda et al. have invented a smart plastic belt capable of steering a pulley system (Figure 2.17). ${ }^{85}$ The smart belt, made of a laminated composite of light-responsive liquid crystal polymer and flexible polyethylene films, was in the form of a closed end film (ring shaped). The belt was placed on the pulley. Utilizing contraction and expansion under light, similar to the bending of open-ended films, the smart belt could roll under the operation of UV and visible light, in turn causing the pulley to rotate continuously. The rotation is due to the presentation of a new site on the films to the 
light path at every turn. Intriguingly, such a smart plastic belt rolling on a wheel can serve as motor system, also demonstrating the transformation of light energy into useful mechanical work.

a

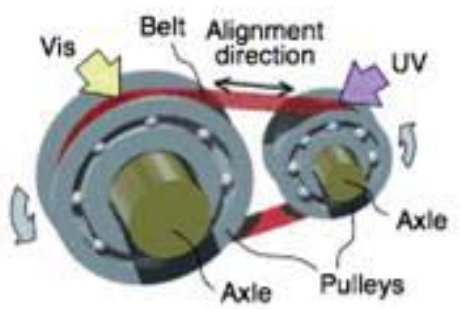

b

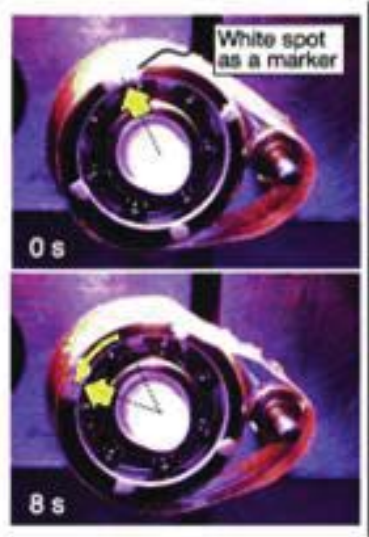

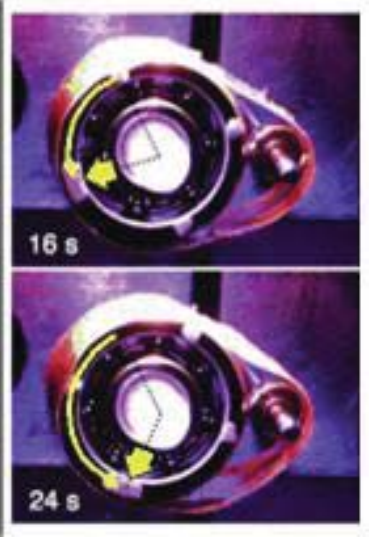

Figure 2.17 a) Model of light-driven plastic motor showing operating site of light and rotational direction. b) Rotation of wheel upon illumination using a s liquid crystal laminated film. Adapted with permission from [85] (Copyright 2008 WILEY-VCH Verlag GmbH \& Co. KGaA, Weinheim).

\subsection{Conclusions}

Harnessing elemental mechanics via the amplification of motion from the molecular level up to the macroscopic level arguably constitutes the most promising strategy to achieve complex, versatile and eventually useful macroscopic motion and deformation of materials. Photo-active molecules are a fascinating source to implement this molecular motion, as light allows information to be delivered rapidly, without contact, and with good precision. Consequently, a variety of molecular level machines have been engineered and synthesized, which transform light into linear, rotary, switch and complex motion, and they have been used to engineer macroscopic mechanical properties such as resizing, bending, twisting and torsion. However, these inspiring examples only hint at the potential of such an approach, ${ }^{89}$ In particular, improvements are expected to affect the controllability of macroscopic motion, the kinetics of motion, its versatility, as well as simplification of the processing of the materials. It may be that someone will perhaps come up with an inventive design of a molecular element or a new approach of structural construction. Learning and adapting from 
Strategies to amplify the light-driven motion of molecules up to the macroscopic level

our master, i.e. Nature, is also crucial in opening the way to success. Eventually, the longterm aim of this research is to find strategies that can be exploited in real-world applications.

\subsection{References}

1 Browne, W. R.; Feringa, B. L. Nat. Nanotechnol. 2006, 1, 25.

2 Wang, J.; Manesh, K. M. Small 2010, 6, 338.

3 Abendroth, J. M.; Bushuyev, O. S.; Weiss, P. S.; Barrett, C. J. ACS Nano 2015.

$4 \quad$ Mattia, E.; Otto, S. Nat. Nanotechnol. 2015, 10, 111.

5 Ikeda, T.; Mamiya, J.-i.; Yu, Y. Angew. Chem. Int. Ed. 2007, 46, 506.

6 Ikeda, T.; Ube, T. Mater. Today 2011, 14, 480.

7 Kim, T.; Zhu, L.; Al-Kaysi, R. O.; Bardeen, C. J. ChemPhysChem 2014, 15, 400.

8 Silvi, S.; Venturi, M.; Credi, A. Chem. Commun. 2011, 47, 2483.

9 Zheng, Y. B.; Hao, Q.; Yang, Y.-W.; Kiraly, B.; Chiang, I. K.; Huang, T. J. J. Nanophotonics. 2010, 4, 042501.

10 Bossi, M. L.; Aramendía, P. F. J Photoch. Photobio. C 2011, 12, 154.

11 Nigel Corns, S.; Partington, S. M.; Towns, A. D. Color. Technol. 2009, 125, 249.

12 Russew, M.-M.; Hecht, S. Adv. Mater. 2010, 22, 3348.

13 Natali, M.; Giordani, S. Chem. Soc. Rev. 2012, 41, 4010.

14 Feringa, B. L.; Browne, W. R. Molecular Switches; Wiley-VCH Verlag GmbH \& Co. KGaA, 2011.

15 Katsonis, N.; Lubomska, M.; Pollard, M. M.; Feringa, B. L.; Rudolf, P. Prog. Surf. Sci. 2007, 82, 407.

16 Robert Resnick, D. H., Kenneth S. Krane Physics; John Wiley \& Sons Inc, 1992.

17 Konstas, K.; Langford, S. J.; Latter, M. J. Int. J. Mol. Sci. 2010, 11, 2453.

18 Yang, W.; Li, Y.; Liu, H.; Chi, L.; Li, Y. Small 2012, 8, 504.

19 Silvi, S.; Venturi, M.; Credi, A. J. Mater. Chem. 2009, 19, 2279.

20 Berna, J.; Leigh, D. A.; Lubomska, M.; Mendoza, S. M.; Perez, E. M.; Rudolf, P.; Teobaldi, G.; Zerbetto, F. Nat. Mater. 2005, 4, 704.

21 Feringa, B. L. Acc. Chem. Res. 2001, 34, 504.

22 Feringa, B. L. J. Org. Chem. 2007, 72, 6635. 
23 Vicario, J.; Walko, M.; Meetsma, A.; Feringa, B. L. J. Am. Chem. Soc. 2006, 128, 5127.

24 Bauer, J.; Hou, L.; Kistemaker, J. C. M.; Feringa, B. L. J. Org. Chem. 2014, 79, 4446.

25 ter Wiel, M. K. J.; van Delden, R. A.; Meetsma, A.; Feringa, B. L. J. Am. Chem. Soc. 2003, 125, 15076.

26 Cnossen, A.; Hou, L.; Pollard, M. M.; Wesenhagen, P. V.; Browne, W. R.; Feringa, B. L. J. Am. Chem. Soc. 2012, 134, 17613.

27 Chen, K.-Y.; Ivashenko, O.; Carroll, G. T.; Robertus, J.; Kistemaker, J. C. M.; London, G.; Browne, W. R.; Rudolf, P.; Feringa, B. L. J. Am. Chem. Soc. 2014, $136,3219$.

28 Muraoka, T.; Kinbara, K.; Kobayashi, Y.; Aida, T. J. Am. Chem. Soc. 2003, 125, 5612.

29 Shirai, Y.; Morin, J.-F.; Sasaki, T.; Guerrero, J. M.; Tour, J. M. Chem. Soc. Rev. 2006, 35, 1043.

30 Zhou, W.; Guo, Y.-J.; Qu, D.-H. J. Org. Chem. 2013, 78, 590.

31 Sasaki, T.; Tour, J. M. Org. Lett. 2008, 10, 897.

32 Irie, M.; Kato, M. J. Am. Chem. Soc. 1985, 107, 1024.

33 Takeshita, M.; Irie, M. Tetrahedron Lett. 1998, 39, 613.

34 Takeshita, M.; Irie, M. J. Org. Chem. 1998, 63, 6643.

35 Takeshita, M.; Soong, C. F.; Irie, M. Tetrahedron Lett. 1998, 39, 7717.

36 Norikane, Y.; Tamaoki, N. Org. Lett. 2004, 6, 2595.

37 Muraoka, T.; Kinbara, K.; Aida, T. Nature 2006, 440, 512.

38 Muraoka, T.; Kinbara, K.; Wakamiya, A.; Yamaguchi, S.; Aida, T. Chem. Eur. J. 2007, 13, 1724.

39 Morin, J.-F.; Shirai, Y.; Tour, J. M. Org. Lett. 2006, 8, 1713.

40 Chiang, P.-T.; Mielke, J.; Godoy, J.; Guerrero, J. M.; Alemany, L. B.; Villagómez, C. J.; Saywell, A.; Grill, L.; Tour, J. M. ACS Nano 2012, 6, 592.

41 Vives, G.; Tour, J. M. Acc. Chem. Res. 2009, 42, 473.

42 Kudernac, T.; Ruangsupapichat, N.; Parschau, M.; Macia, B.; Katsonis, N.; Harutyunyan, S. R.; Ernst, K.-H.; Feringa, B. L. Nature 2011, 479, 208. 
Strategies to amplify the light-driven motion of molecules up to the macroscopic level

43 Nguyen, S. T.; Gin, D. L.; Hupp, J. T.; Zhang, X. Proc. Natl. Acad. Sci. 2001, 98, 11849.

44 van Herpt, J. T.; Areephong, J.; Stuart, M. C. A.; Browne, W. R.; Feringa, B. L. Chem. Eur. J. 2014, 20, 1737.

45 Mba, M.; Mazzier, D.; Silvestrini, S.; Toniolo, C.; Fatás, P.; Jiménez, A. I.; Cativiela, C.; Moretto, A. Chem. Eur. J. 2013, 19, 15841.

46 Bushuyev, O. S.; Singleton, T. A.; Barrett, C. J. Adv. Mater. 2013, 25, 1796.

47 Bushuyev, O. S.; Tomberg, A.; Friščić, T.; Barrett, C. J. J. Am. Chem. Soc. 2013, $135,12556$.

48 Morimoto, M.; Irie, M. J. Am. Chem. Soc. 2010, 132, 14172.

49 Rogovina, L. Z.; Vasil’ev, V. G.; Braudo, E. E. Polym. Sci. Ser. C 2008, 50, 85.

50 van Herpt, J. T., Groningen, 2013.

51 Zhang, L.; Naumov, P. Angew. Chem. Int. Ed. 2015, 54, 8642.

52 Li, Q.; Fuks, G.; Moulin, E.; Maaloum, M.; Rawiso, M.; Kulic, I.; Foy, J. T.; Giuseppone, N. Nat. Nanotechnol. 2015, 10, 161.

53 Zhang, L.; Liang, H.; Jacob, J.; Naumov, P. Nat. Commun. 2015, 6.

54 Bai, Y.; Hu, J.; Shen, W.; Hu, X. Polym. Int. 2014, 63, 1539.

55 Wei, J.; Yu, Y. Soft Matter 2012, 8, 8050.

56 Iqbal, D.; Samiullah, M. Materials 2013, 6, 116.

57 White, T. J.; Broer, D. J. Nat. Mater. 2015, 14, 1087.

58 Fleischmann, E.-K.; Zentel, R. Angew. Chem. Int. Ed. 2013, 52, 8810.

59 Finkelmann, H.; Nishikawa, E.; Pereira, G. G.; Warner, M. Phys. Rev. Lett. 2001, 87,015501 .

60 Yu, Y.; Nakano, M.; Ikeda, T. Nature 2003, 425, 145.

61 van Oosten, C. L.; Bastiaansen, C. W. M.; Broer, D. J. Nat. Mater. 2009, 8, 677.

62 Dierking, I. Textures of liquid crystal; Wiley-VCH Verlag GmbH \& co. KGaA, 2003.

63 de Haan, L. T.; Schenning, A. P. H. J.; Broer, D. J. Polym. J. 2014, 55, 5885.

64 Yu, Y.; Ikeda, T. Angew. Chem. Int. Ed. 2006, 45, 5416.

65 Palffy-Muhoray, P. Nat. Mater. 2009, 8, 614.

66 Corbett, D.; Warner, M. Liq. Cryst. 2009, 36, 1263. 
67 Liu, D.; Broer, D. J. Nat. Commun. 2015, 6.

68 Yu, H. J. Mater. Chem. C 2014, 2, 3047.

69 Warner, M. Phys. Rev. Lett. 2004, 92, 134302.

$70 \quad$ Kondo, M.; Yu, Y.; Ikeda, T. Angew. Chem. Int. Ed. 2006, 45, 1378.

71 van Oosten, C. L.; Harris, K. D.; Bastiaansen, C. W. M.; Broer, D. J. Eur. Phys. J. E 2007, 23, 329.

72 White, T. J.; Tabiryan, N. V.; Serak, S. V.; Hrozhyk, U. A.; Tondiglia, V. P.; Koerner, H.; Vaia, R. A.; Bunning, T. J. Soft Matter 2008, 4, 1796.

73 Serak, S.; Tabiryan, N.; Vergara, R.; White, T. J.; Vaia, R. A.; Bunning, T. J. Soft Matter 2010, 6, 779.

74 Lee, K. M.; White, T. J. Macromolecules 2012, 45, 7163.

75 Yoshino, T.; Kondo, M.; Mamiya, J.-i.; Kinoshita, M.; Yu, Y.; Ikeda, T. Adv. Mater. 2010, 22, 1361.

76 Wu, W.; Yao, L.; Yang, T.; Yin, R.; Li, F.; Yu, Y. J. Am. Chem. Soc. 2011, 133, 15810.

77 Jiang, Z.; Xu, M.; Li, F.; Yu, Y. J. Am. Chem. Soc. 2013, 135, 16446.

78 Mamiya, J.-i.; Kuriyama, A.; Yokota, N.; Yamada, M.; Ikeda, T. Chem. Eur. J. 2015, 21, 3174.

79 Priimagi, A.; Barrett, C. J.; Shishido, A. J. Mater. Chem. C 2014, 2, 7155.

$80 \quad$ Forterre, Y.; Dumais, J. Science 2011, 333, 1715.

81 Harris, K. D.; Cuypers, R.; Scheibe, P.; van Oosten, C. L.; Bastiaansen, C. W. M.; Lub, J.; Broer, D. J. J. Mater. Chem. 2005, 15, 5043.

82 Lee, K. M.; Smith, M. L.; Koerner, H.; Tabiryan, N.; Vaia, R. A.; Bunning, T. J.; White, T. J. Adv. Funct. Mater. 2011, 21, 2913.

83 Wie, J. J.; Lee, K. M.; Smith, M. L.; Vaia, R. A.; White, T. J. Soft Matter 2013, 9, 9303.

84 Tabiryan, N.; Serak, S.; Dai, X.-M.; Bunning, T. Opt. Express 2005, 13, 7442.

85 Yamada, M.; Kondo, M.; Mamiya, J.-i.; Yu, Y.; Kinoshita, M.; Barrett, C. J.; Ikeda, T. Angew. Chem. Int. Ed. 2008, 47, 4986.

86 Sugiura, S.; Sumaru, K.; Ohi, K.; Hiroki, K.; Takagi, T.; Kanamori, T. Sensor. Actuat. A-Phys. 2007, 140, 176. 
Strategies to amplify the light-driven motion of molecules up to the macroscopic level

87 ter Schiphorst, J.; Coleman, S.; Stumpel, J. E.; Ben Azouz, A.; Diamond, D.; Schenning, A. P. H. J. Chem. Mater. 2015.

88 Cheng, F.; Yin, R.; Zhang, Y.; Yen, C.-C.; Yu, Y. Soft Matter 2010, 6, 3447.

89 Peplow, M. Nature 2015, 525, 18. 


\section{Chapter 3}

\section{Bio-inspired strategies to convert light into macroscopic twist motion}

A key goal of nanotechnology is the development of artificial machines capable of converting molecular movement into macroscopic work. Although conversion of light into shape changes has been reported and compared to artificial muscles, real applications require work against an external load. Here, we describe the design, synthesis and operation of spring-like materials capable of converting light energy into mechanical work at the macroscopic scale. These versatile materials consist of molecular switches embedded in liquid-crystalline polymer springs. In these springs, molecular movement is converted and amplified into controlled and reversible twisting motions. The springs display complex motion, which includes winding, unwinding and helix inversion, as dictated by their initial shape. Importantly, they can produce work by moving a macroscopic object and mimicking mechanical movements, such as those used by plant tendrils to help the plant access sunlight. These functional materials have potential applications in micromechanical systems, soft robotics and artificial muscles.

This chapter was published in: S. Iamsaard, S. J. Aßhoff, B. Matt, T. Kudernac, J. J. L. M. Cornelissen, S. P. Fletcher and N. Katsonis, Nat. Chem. 2014, 6, 229-235. Part of the chapter was included in a submission to Nature Protocols. 


\subsection{Introduction}

The promise of developing artificial molecular machines has inspired scientists for decades $^{1,2}$ and led to the design and operation of small molecules that exhibit mechanically relevant movements, such as rotation, ${ }^{3-6}$ walking ${ }^{7}$ and twisting, ${ }^{8}$ as well as complex molecular-scale operations, such as driving ${ }^{9}$ and chemical synthesis. ${ }^{10}$ Rudimentary examples of nanomachines capable of work ${ }^{11-15}$ provide tantalizing hints of the potential power of molecular devices. However, materials capable of translating molecular motion into useful macroscopic function remain elusive. ${ }^{16-19}$

Nature uses molecular-scale machines to drive every significant biological process ${ }^{20}$ and powers macroscopic mechanical motion in plants in highly complex processes. ${ }^{21}$ Examples of biological systems built on helical motion include powerful engines such as spasmoneme springs, ${ }^{21}$ seed pod opening ${ }^{22}$ and tendril coiling. ${ }^{23}$

Based on the general concept that such plant-like helical deformations may similarly occur in artificial systems, which thus makes them capable of producing mechanical work, we set out to design a system in which molecular movement would be translated across scale lengths into macroscopic rotational movement, and form the basis of functional materials. Previous studies reported on a light-induced film twisting of thin crystals ${ }^{24,25}$ and out of plane deformations of polymer films controlled by irradiation with polarized light. ${ }^{26}$ In these examples, selective irradiation was necessary to control the twisting mechanism. In contrast, here we seek to create smart materials with photoresponses inherently encoded in their structure.

\subsection{Results and discussion}

\subsubsection{Amplifying molecular chirality in a liquid-crystal polymer}

Our approach involves using a liquid-crystal polymer network ${ }^{27}$ that can selectively form either right-handed or left-handed macroscopic helices at room temperature. Liquid-crystal polymer networks display a strong coupling between orientational order and mechanical strain, which is why they undergo deformations when submitted to a stimulus-induced decrease of order. Light is an especially attractive stimulus to induce such macroscopic 
modifications, as it has proved to be a versatile and controllable energy source for driving a wide range of nanoscale molecular systems. ${ }^{11,12,15-18}$ Arguably, the most straightforward manner to induce light responsiveness in a polymer network is to introduce light-responsive switches, such as azobenzene units, ${ }^{28-30}$ into its covalent structure. Molecular switch 1 (Figure $3.1 \mathrm{a})$ is an azobenzene-based photochromic switch that undergoes trans-cis photoisomerization under irradiation with ultraviolet light (see Section 3.5.2 Figure 3.11). The switch acts as a nanoscale energy converter and is incorporated into the polymer network through polymerizable acrylate groups. It has been shown that for azobenzene concentrations up to $10 \mathrm{wt} \%$, photoinduced deformations scale with the concentration of the azobenzene, ${ }^{31}$ and thus the concentration of $\mathbf{1}$ in the polymer network was set to $10 \mathrm{wt} \%$ to provide large photoinduced deformations.

The host matrix we used is based on a combination of a low molecular-weight nematic liquid crystal (commercially available E7) and acrylate-functionalized nematic liquid crystals that photopolymerize in the presence of an initiator (see Section 3.5.1; Figure 3.10). We selected a photoinitiator that absorbs at wavelengths longer than $420 \mathrm{~nm}$ to achieve crosspolymerization while minimizing the isomerization of $\mathbf{1}$. A small amount of the chiral dopant (S811, Figure 3.1b) was added to the liquid-crystalline mixture to favour a left-handed twist (Figure 3.1c). The mixture was then introduced into a glass cell to promote a twist geometry, in which the orientation of the liquid-crystal director changes smoothly by $90^{\circ}$ from the bottom surface to the top surface (Figure 3.1c and d). Polarized optical microscopy shows that the molecular organization of the liquid crystal is largely preserved after polymerization. After the cells were opened and the films dried, strips with a width of $0.7-0.9 \mathrm{~mm}$ were cut in a direction characterized by the angular offset $\varphi$ defined here as the angle between the orientation of the molecules at mid-plane on one hand, and the cutting direction on the other hand, which also corresponds to the long axis of the ribbons (Figure 3.1c and 3.2). An illustration of how the films are cast and how they coil spontaneously into springs once they are cut into ribbons is provided in section 3.5.3 (Figure 3.13). Below we show that the direction in which the ribbons are cut is a parameter that determines not only the pitch and handedness of the helical shapes that are formed, but also their photoresponsive behaviour. 
Bio-inspired strategies to convert light into macroscopic twist motion

$\mathrm{a}$

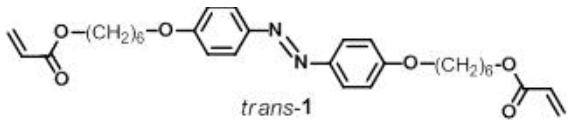

1f $\mathrm{hv}=365 \mathrm{~nm}$

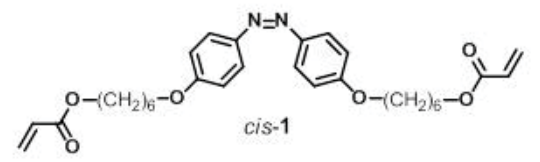

c

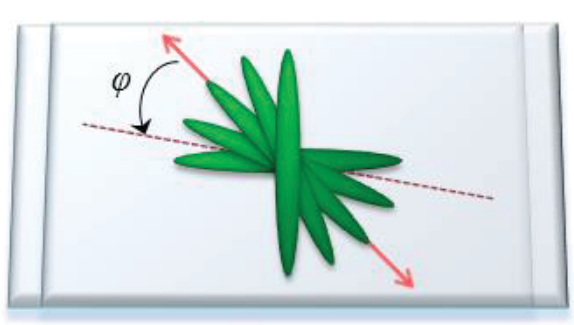

$\mathrm{b}$

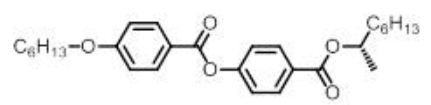

S-811

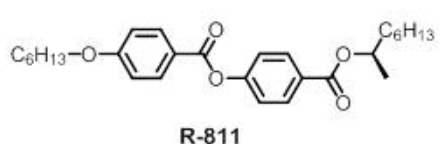

d

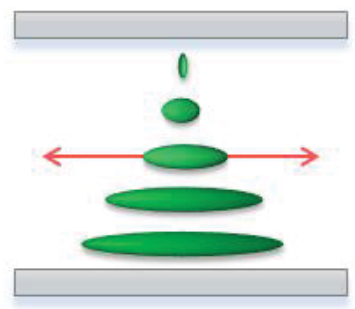

Figure 3.1 A photoresponsive liquid crystal in a twist-nematic molecular organization. a) The molecular photoswitch $\mathbf{1}$ used in this study is an azobenzene derivative. b) Chiral dopants S811 and R811 induce a left-handed and right-handed twist in the liquid crystal, respectively. c) Molecular organization in the twist cell (top view) and the angular offset $\varphi$, which characterizes the angle at which the ribbon is cut. The orientation of the molecules at midplane is shown with a double-headed arrow. The cutting direction, which is also the long axis of the ribbon, is represented by a dotted line. The elongated rods represent molecules. d) The twist-nematic molecular orientation through the thickness of the film (side view).

\subsubsection{Generating a diversity of helical shapes}

On polymerization and/or drying of such a film, anisotropic shrinking occurs and leads to out of plane twisting once the film is released from the cell and cut into ribbons. ${ }^{32-34}$ In particular, Urayama and co-workers presented a strain model according to which ribbons cut out from twisted nematics select a helicoid or spiral shape, depending on their width. ${ }^{32}$ That is, polymerization and/or drying of the ribbons induces shrinking of each face of the ribbon orthogonally to each other, and the ribbons have to curl to accommodate for it. The shrinking is anisotropic, so the shape of each ribbon is determined by the orientation of the molecules with respect to the short and long axes of the ribbons.

In the work reported here, the width of the ribbons was constant. We propose that the diversity of helical shapes we observed arises from three sources, which can reinforce or compete depending on the angle at which the ribbon is cut: (1) the handedness of the director twist, which is determined by the handedness of the chiral dopant, (2) the angular offset $\varphi$, 
which is determined by the angle at which the ribbon is cut, and (3) a gradient in density is expected through the thickness of the sample, because the films are always irradiated from the same side, that is from the top of the sample, during photopolymerization. Based on the competition between these sources of asymmetry, the samples can form a rich variety of chiral shapes, from left-handed or right-handed ribbons with diverse pitches (Figure 3.2, ribbons $\mathrm{B}$ and $\mathrm{D}$ ), to ribbons for which chirality is not expressed macroscopically (Figure 3.2, flat ribbon A or open-ring ribbon $\mathrm{C}$ ). Additional images for the shape selection of the ribbons are shown in Section 3.5.4 (Figure 3.16 and 3.17).

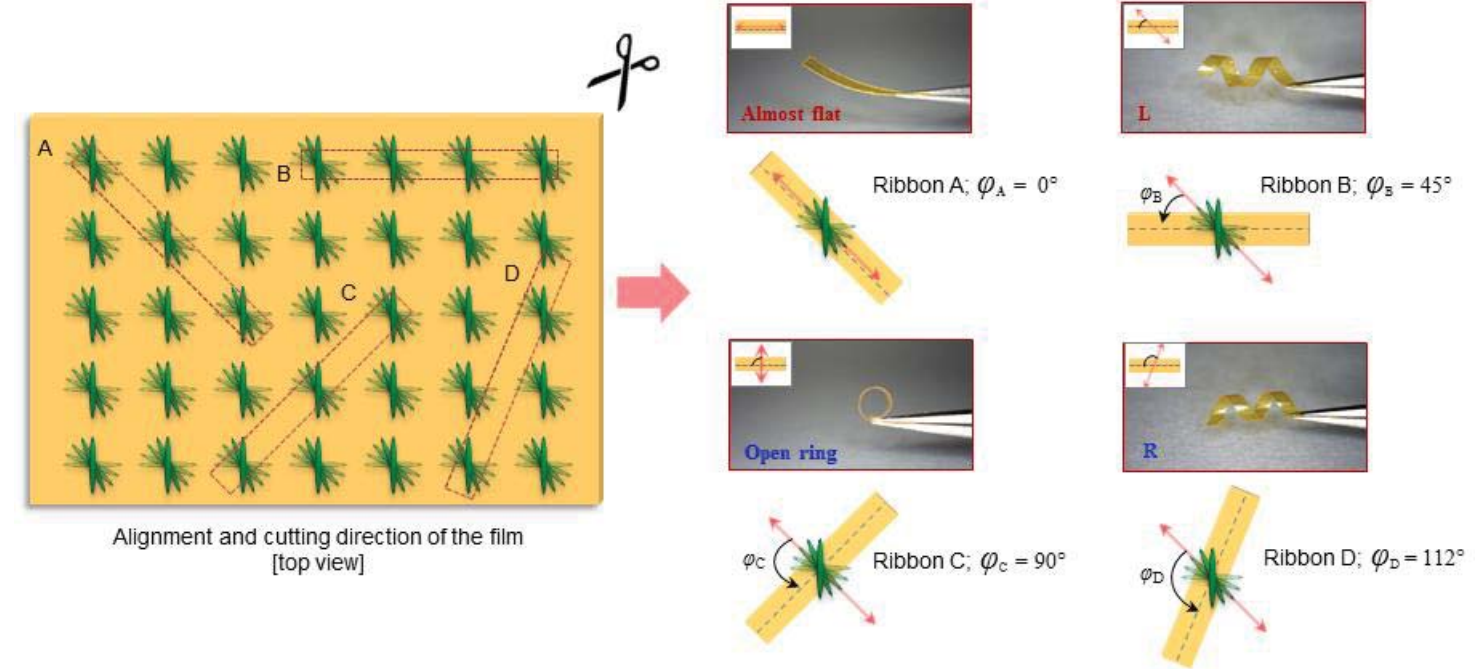

Figure 3.2 The ribbons display a variety of shapes that depend on the direction in which they are cut. The samples display a rich variety of chiral shapes, from left-handed or right-handed ribbons (ribbons $\mathrm{B}$ and $\mathrm{D}$ ) to ribbons for which chirality is not expressed macroscopically (flat ribbon $\mathrm{A}$ or open-ring ribbon $\mathrm{C}$ ).

As expected, in ribbons where the chiral dopant $\mathbf{S 8 1 1}$ was replaced by its enantiomer R811 we observed that the shape of the ribbons was the mirror image - that is, the polymers produced were enantiomers of each other (Figure 3.3 and see also Section 3.5.4; Figure 3.16 and 3.17). For example, an $\mathbf{S 8 1 1}$ ribbon cut at $\varphi=22^{\circ}$ is expected to be the enantiomer of an $\mathbf{R 8 1 1}$ ribbon cut at $\varphi=158^{\circ}$ (Figure 3.3), as both the handedness of the director twist and the angular offset are mirror images in these two ribbons. Indeed, we observed that the former ribbon yielded a left-handed spring, whereas the latter yielded a right-handed spring of similar pitch (Section 3.3). Diastereomeric ribbons were also examined (Figure 3.3 and see also Section 3.5.4; Figure 3.16 and 3.17). 

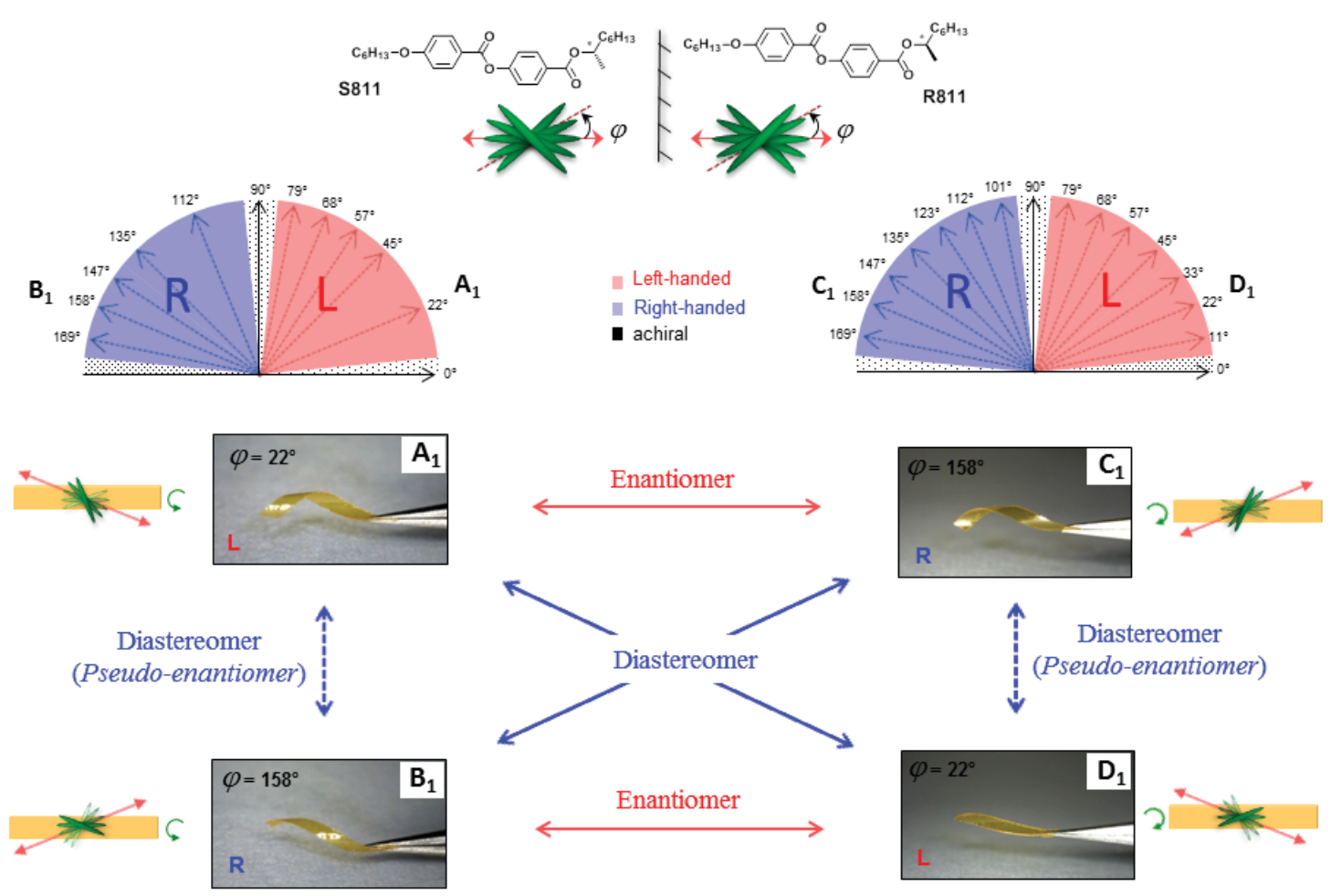

Figure 3.3 Shape of polymer springs as function of angular offset and illustration of their stereoisomer, indicating relation of macroscopic shapes with respect to molecular geometry. (Left-side) Ribbons doped with S811, in which the director twist is left-handed; (Right-side) Ribbons doped with R811, where the director twist is right-handed.

In passive (non-photoactive) ribbons that have been emptied from chiral molecules and non-reacted materials, twist-free ribbons (either open-ring ribbons or flat ribbons) have been observed at $\varphi \approx 45^{\circ}$ and $135^{\circ}$, which is expected for a non-chiral system of orthogonally shrinking sheets. ${ }^{35}$ In contrast, we observed achiral ribbons for $\varphi \approx 0^{\circ}$ and $90^{\circ}$, where the surface directors are not parallel to the axes of the ribbon but, instead, the mid-plane director is parallel to one of the axes. This difference indicates a strong source of asymmetry in the system. To verify this conclusion, we heated the ribbons to $180{ }^{\circ} \mathrm{C}$ (Figure 3.4 and Section 3.5.4; Figure 3.18). As the temperature was gradually increased, unreacted materials (E7 and chiral dopant) were expelled from the polymer network. When all these materials had diffused away, the ribbons were emptied from the additional source of asymmetry provided by $\mathbf{S 8 1 1}$ and underwent a shape transition. At $180{ }^{\circ} \mathrm{C}$, the shapes we observed corresponded to the shapes of the passive ribbons observed by Sawa et al. ${ }^{32,33}$; in particular, the shape of 
the $\varphi=45^{\circ}$ ribbon became achiral. Importantly, the temperature-induced shape transition that we observed was not reversible.
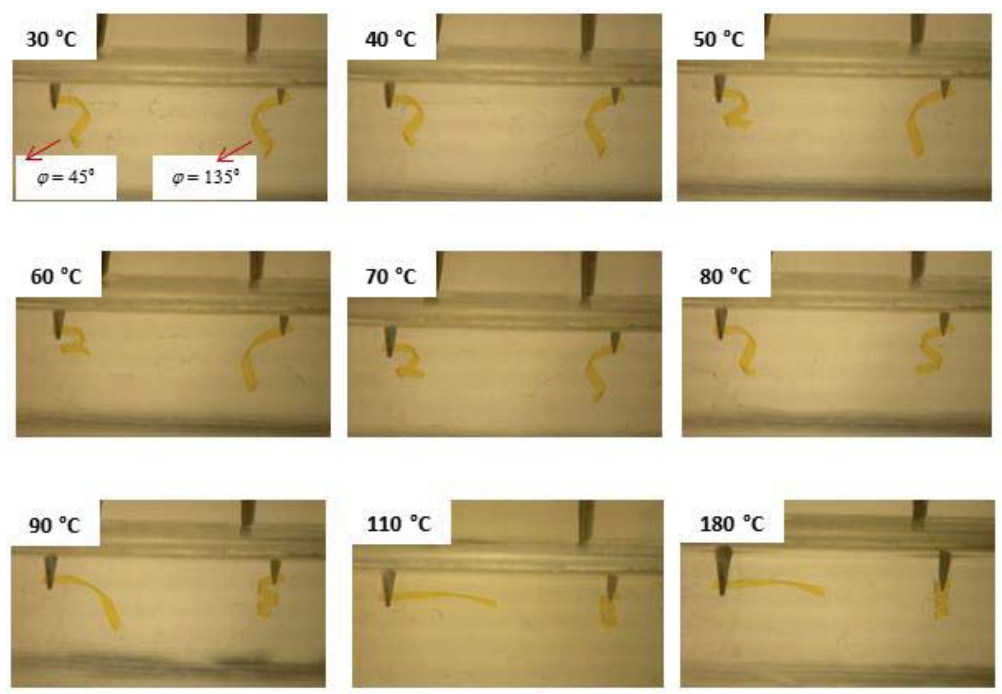

Figure 3.4 Thermoresponsive behavior of the ribbons doped with S811.

Surprisingly, after cutting, all the ribbons presented here curled in such a way that the area facing the top of the cell was always found on the outside of the ribbon, whereas the bottom of the film always ended up inside the twisted ribbon. This conclusion was drawn after labelling one side of the ribbons with sticky tape (Section 3.5.4; Figure 3.19 and 3.20). We propose that the preferential curling direction originates in a light-intensity gradient caused by the thickness of the sample during photopolymerization, because both the photoactive dopant and the photoinitiator are optical absorbers at $\lambda \geq 420 \mathrm{~nm}$ (Section 3.5.2; Figure 3.11). As the polymerization rate scales with light intensity, the reactive monomers are depleted preferentially at the top of the sample. This gradient of concentration in reactive monomers drives the diffusion of the monomers to the top of the film during polymerization and the final sample displays a higher density and cross-link density at the top. ${ }^{36}$ To confirm that the curvature of the ribbons is influenced by a density gradient in the film, we performed photopolymerization by placing the lamp at the bottom of the sample (rather than at its top). For a fixed offset angle, we observed that the ribbons had a similar pitch, but an opposite handedness. We found that when photopolymerization occurred from the bottom, the bottom of the film always ended up outside the spiral ribbons. This observation provides insight into 
both the shape and the photoresponsive properties of the springs. In all the experiments described below, the ribbons were prepared by photopolymerization from the top of the cell.

To summarize, two parameters characterize the shape of the ribbons - the pitch and the bending direction. The pitch of the spiral ribbons (that is, the absolute value of the curvature) is determined by the director orientation within the ribbons. The bending direction (that is, the handedness of the spiral ribbons) is determined not only by the director orientation within the ribbons, but also by the direction of the polymer gradient.

\subsubsection{Photoactuation modes of the helical ribbons}

We investigated the photoresponse of these twisted ribbons (Figure 3.5 and 3.6). Under irradiation with light, left-handed spiral ribbons doped with $\mathbf{S 8 1 1}$ decreased in their macroscopic pitch, and the corresponding right-handed ribbons showed an increase in macroscopic pitch. Remarkably, it was also possible to observe inversion of the helical sense from right-handed to left-handed (Figure 3.5 and 3.6a). After a few minutes the deformations

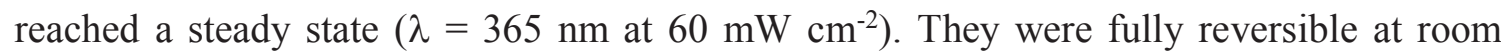
temperature, with relaxation times of the order of a dozen minutes. With visible light, this reversal process could be accelerated to completion in a few seconds. The winding of the photoresponsive ribbons was associated with remarkably large isochoric contraction (which reached up to about $60 \%$ of the original length of the ribbons). For unwinding associated with elongation, amplitudes of up to $40 \%$ of the original shape were observed (Figure 3.6b). The direction of the actuation (expansion or contraction) and its magnitude are both encoded in the shape and geometry of each spiral ribbon. 

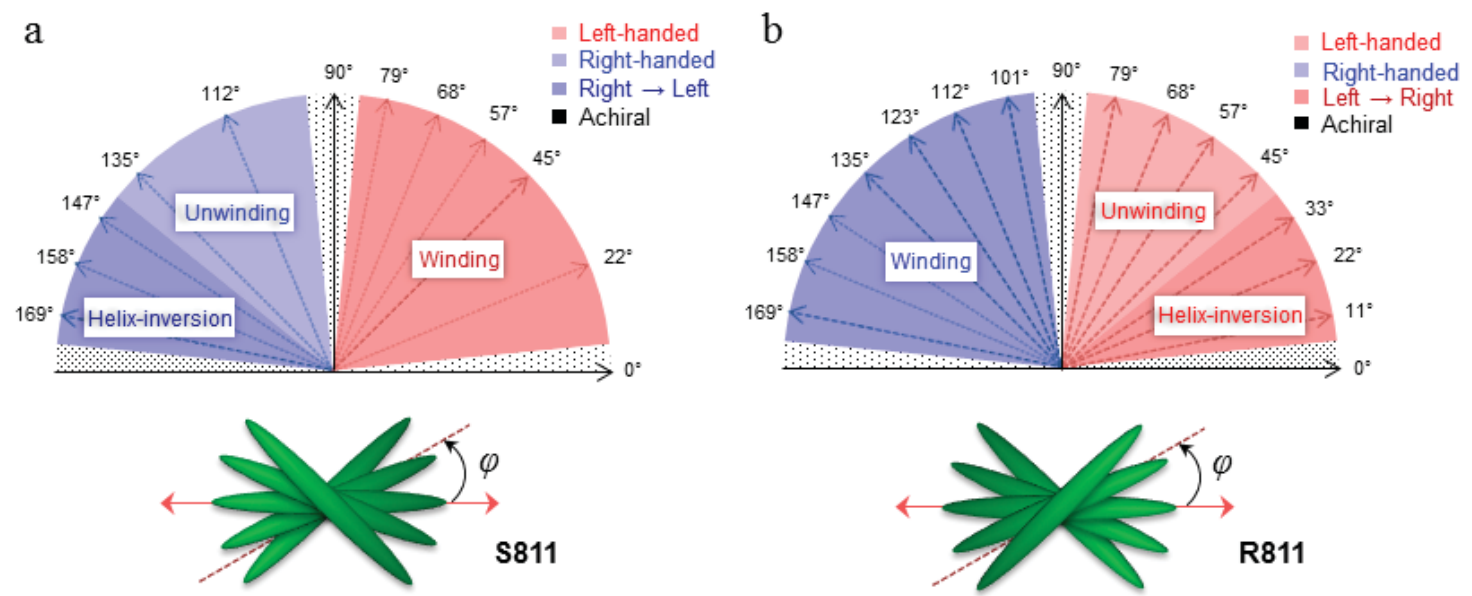

Figure 3.5 Shape and photoactuation modes of the polymer springs as a function of the angular offset. The angular offset $\varphi$ is defined here as the angle between the orientation of the molecules at mid-plane and the cutting direction. The experimental error is estimated to $\pm 2^{\circ}$. a) Ribbons doped with S811, in which the director twist is left-handed. b) Ribbons doped with R811, where the director twist is right-handed.

Important characteristics of an actuator are rate of response and reversibility. In the case of photoisomerization reactions, the intensity of irradiation constitutes a control parameter for reaction rate. However, it is probable that the rate of the shape reversal is determined by the rate of thermal relaxation of the photoactive dopant, the cis-azobenzene molecule. Here, the half-life time for thermal relaxation of the cis-azobenzene was about 15 minutes in solution and under ambient light (see Section 3.5.2 Figure 3.11). This half-life corresponds well to the thermal relaxation rate of the ribbons, which suggests that the reorganization of the polymer network is faster than the thermal isomerization of the cis-azobenzene. Thus, the relaxation process is probably determined by the specific molecular structure of the photoresponsive switch. The thermal relaxation can be accelerated by irradiation with visible light. 
Bio-inspired strategies to convert light into macroscopic twist motion
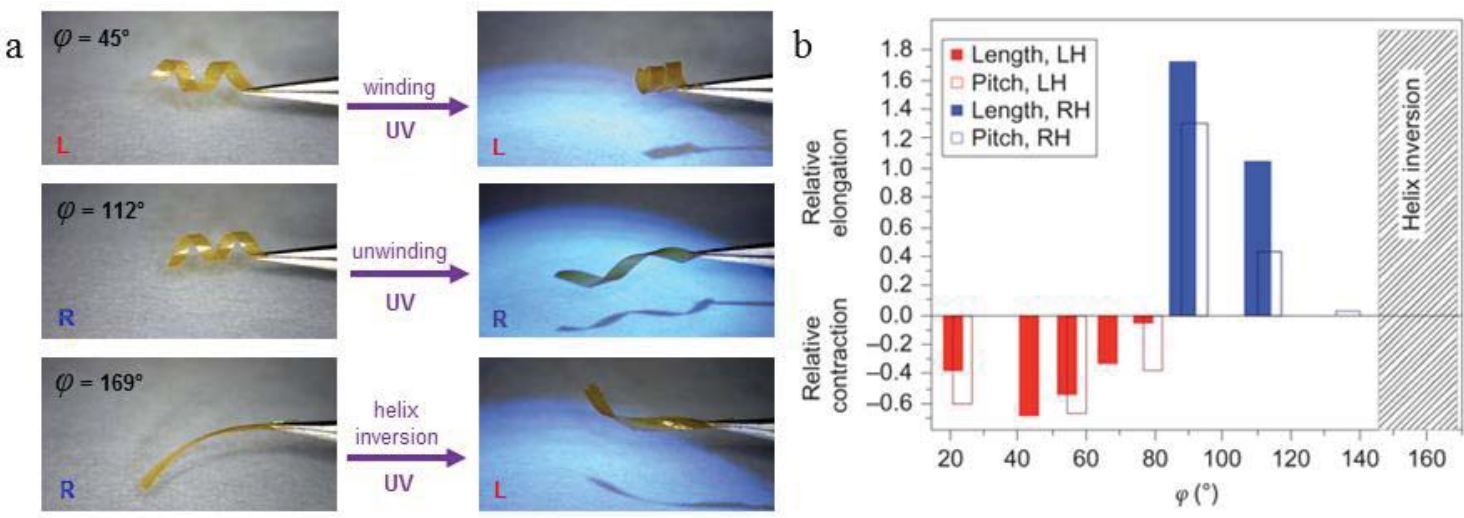

c

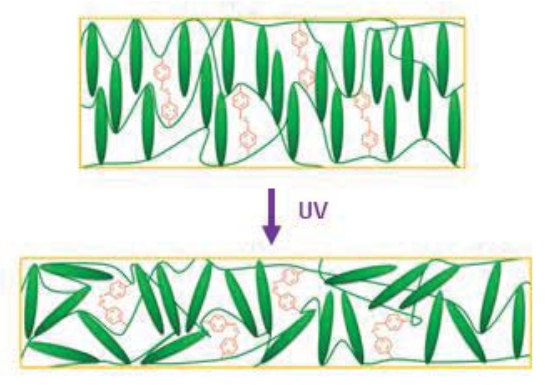

d

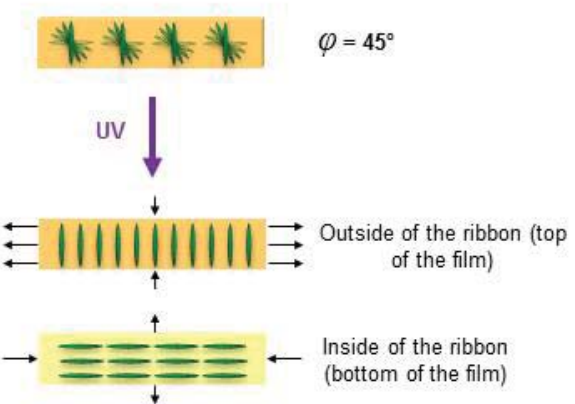

Figure 3.6 Photoactuation modes of the polymer springs doped with S811. a) Spiral ribbons irradiated for two minutes with ultraviolet light $(\lambda=365 \mathrm{~nm})$ display isochoric winding, unwinding and helix inversion as dictated by their initial shape and geometry. b) A large amplitude contraction is obtained on light-driven winding of ribbons with $\varphi \approx 45^{\circ}$, and a large amplitude elongation is obtained on light-driven unwinding of ribbons with $\varphi \approx 90^{\circ}$. LH, left-handed; RH, right-handed. c) Under irradiation with ultraviolet light, the ribbons contract along the director and expand in the perpendicular directions, as is consistent with an ultraviolet-induced increase of disorder. d) Scheme to represent the mechanisms through which the shape of the ribbons is modified under irradiation with ultraviolet light, for $\varphi \approx$ $45^{\circ}$. The ribbons deform to accommodate the preferred distortion along the main axis of the ribbon, and this preferred distortion is determined by the orientation of the molecules.

We also performed absorption spectroscopy of the films on repeated alternating irradiation with ultraviolet light $(\lambda=365 \mathrm{~nm})$ and visible light $(\lambda \geq 420 \mathrm{~nm})$. Photoswitching of azobenzene 1 is, indeed, reversible, even after being covalently bonded to the polymer network, and there was nearly no fatigue effect on the sample after ten cycles of alternating ultraviolet and visible irradiation (see Section 3.5.4; Figure 3.21).

The mechanical behaviour of the springs depends on the handedness of the director twist and on their angular offset $\varphi$ (Figure 3.5). Earlier studies demonstrated that controlling the geometry of the director and its spatial variation in photoresponsive polymer networks can drive various types of light-induced deformations in these materials. ${ }^{26,30}$ Here we show that 
large deformations are possible by utilizing the twisting mechanism. Understanding this mechanism requires that the specificity of light as a stimulus needs to be taken into account. Both experimental and theoretical investigations have established that irradiating liquidcrystal polymer networks doped with photoresponsive molecules induces disorder and thus leads to anisotropic deformations characterized by contraction in the direction of the director, and expansion in perpendicular directions (Figure 3.6c). ${ }^{37}$ As mentioned above, all ribbons curl in such a way that the region that faces the top of the cell during polymerization is always found at the outside of the ribbon, whereas the bottom of the film always ends up inside the twisted ribbon. Having determined the orientation of the molecules on each side of the ribbon before irradiation, an interpretation becomes possible: the ribbons always deform to accommodate the preferred distortion along the main axis of the ribbon, and this preferred distortion is determined by the orientation of the molecules, which is, in turn, determined by the cutting direction.

The mechanism that drives the photoresponse in the ribbons is illustrated for the ribbon where $\varphi=45^{\circ}$ (Figure 3.6d). Here, the outside face undergoes expansion along its long axis, whereas the inner face shrinks. Both photodeformations accommodate an increase in the twist, as observed experimentally (Figure 3.6a). The work provided by such a ribbon can be estimated by attaching a known mass to one of the springs. Under irradiation, the ribbon works against gravity by lifting the mass up (Figure 3.7). This experiment yielded a value of $3.04 \mathrm{~J} \mathrm{~mol}^{-1}$ of azobenzene (and a power of $6 \mathrm{nW}$ ), a similar value to that reported for the work by thin molecular crystals $\left(2.95 \mathrm{~J} \mathrm{~mol}^{-1}\right.$ of a photoresponsive molecule embedded in the crystal; ${ }^{15}$ see section 3.5.4 for the calculation). The similarity between these values highlights that these soft actuators can perform at least as well as their rigid counterparts.

To summarize, the photoresponse of the ribbons at the macroscopic level is determined by the orientation of the molecules within the ribbons. Under irradiation with ultraviolet light, the ribbons contract along the director and expand in the perpendicular directions, as is consistent with an ultraviolet-induced increase of disorder. This mechanism is reminiscent of a design strategy implemented successfully in other photomechanical soft materials: In Aida and co-worker's polymer brushes, irradiation with light unbalances competing strains between the front and back sides of the film, which in turn bends the film. ${ }^{38}$ 


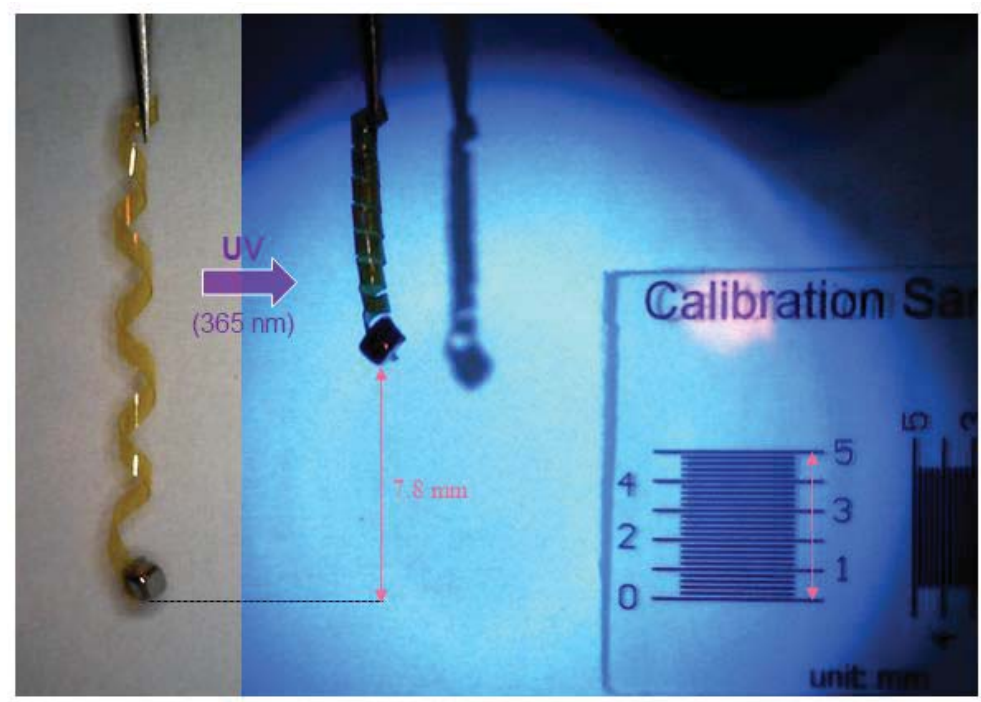

Figure 3.7 An estimate of the work performed by a ribbon doped with $\mathbf{S 8 1 1}\left(\varphi=45^{\circ} ; 0.93\right.$ $\mathrm{mg}$ ) was obtained by fixing a magnet $(7.07 \mathrm{mg})$ to one of its ends. Under irradiation with UV light $\left(\approx 60 \mathrm{~mW} / \mathrm{cm}^{2}\right)$ the ribbon lifts the weight by $7.8 \mathrm{~mm}$ in $\mathrm{t}=90 \mathrm{~s}$.

\subsubsection{Mimicking the mechanical behaviour of plant tendrils}

In the movements of plant tendrils, winding and unwinding is governed by the shrinking and expansion of cell walls orthogonally to each other. In our system, we anticipate that the mechanism of winding and unwinding results from photoisomerization of embedded units of 1 from the trans-state to the bent cis-state, which contracts the material along and elongates it perpendicular to the director. This proposed mechanism bears considerable resemblance to that believed to occur in biological systems.

In nature, tendrils combine bending and twisting distortion modes to find a support and lift a plant upwards, towards the Sun. A fully operational device cut out of a single sheet of a liquid-crystal polymer network was found to display the type of complex and biologically relevant behaviour that inspired the current approach (Figure 3.8). These mixed-helicity springs, which comprise two oppositely handed helices joined at a kink, displayed regions that simultaneously coiled and uncoiled under irradiation (Figure 3.8b-d). Noticeably, when a hybrid spring was clamped at both ends and pulled axially, it unwound. Furthermore, localized irradiation of specific sections of these springs led to a bending motion, the direction of which can be also controlled (Figure 3.8e). 
a

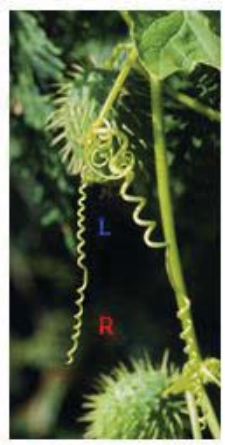

$\mathrm{c}$

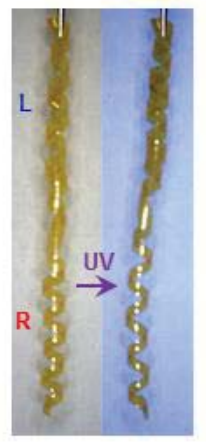

d

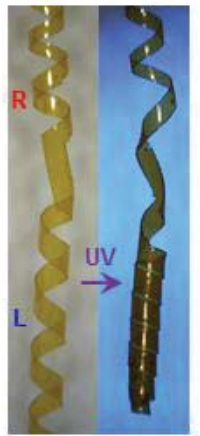

b

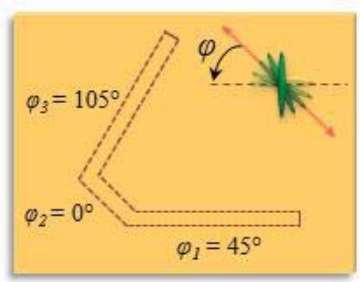

e

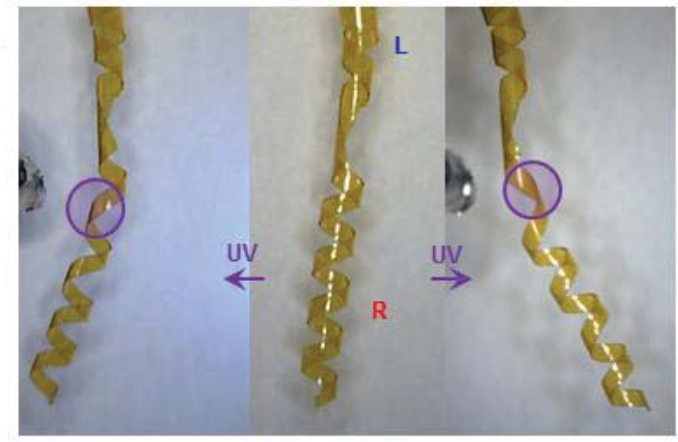

Figure 3.8 Mixed-helicity springs doped with S811 display a complex range of mechanical photoresponses. a) A coiled tendril of the wild cucumber plant. (Image courtesy of Green Thumb Photography/Beth Hoar) b) The liquid-crystal polymer film is cut to introduce regions that display different dynamic behaviours. c,d) A polymer spring that displays a cucumber tendril-like shape, composed of two oppositely handed helices connected by a kink. On irradiation the right-handed helix unwinds (c) and the left-handed helix winds (d). e) Bending of the spring is achieved and controlled by selective irradiation, which induces a local elongation of the right-handed ribbon. The coloured circles indicate the irradiation spots where elongation occurs.

Finally, the differential behaviour of a mixed-helicity spring was used to demonstrate proof-of-principle for a photomechanical engine that performs a complex mechanical function. To this end, a tendril-like spring was clamped at each end and irradiated alternately with ultraviolet and visible light (Figure 3.9). The confinement of the tendril allows the central kink to perform a continuous push-pull motion that is similar to the piston motion essential to the design and functioning of classical mechanical motors. The movement of the spring retained its amplitude over ten cycles (Figure 3.9b). As a further demonstration of the ability of these materials to produce useful work we attached a simple magnet to the central kink of a tendril-like spring. The material was found to withstand the weight of the magnet ( $\mathrm{m} \approx 2 \mathrm{mg}$ ) with only a small reduction in mobility, and the movement of this magnet was 
used to move an additional magnet ( $\mathrm{m} \approx 0.5 \mathrm{mg}$ ), separated (by $10 \mathrm{~mm}$ ) through a glass plate, back and forth in a predictable way that was controlled completely by the mechanical action of the mixed-helicity spring (Figure 3.9c).
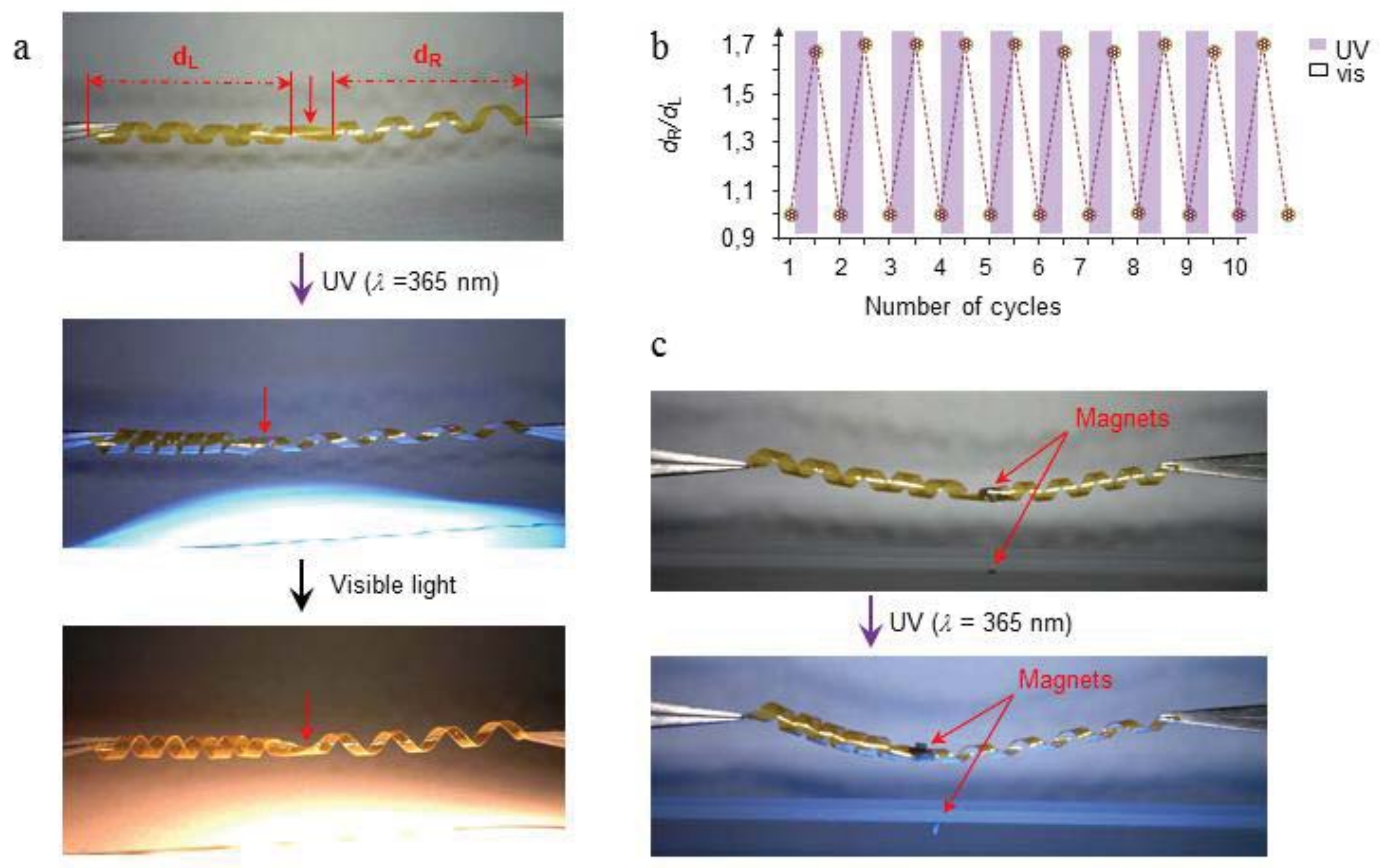

c
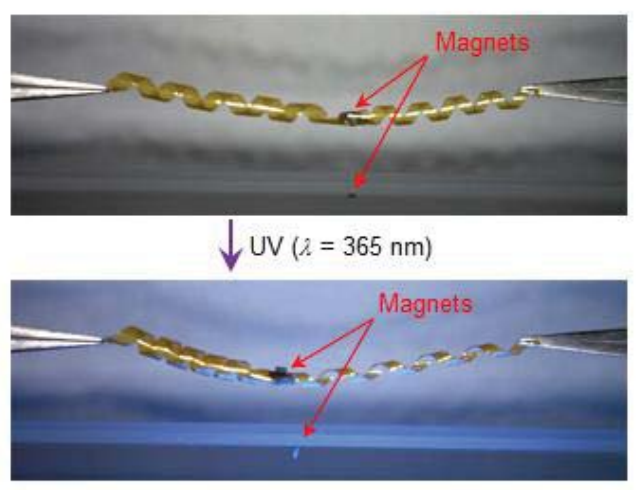

Figure 3.9 Proof-of-principle for a mechanical device powered by light. a) On alternate irradiation with ultraviolet and visible light, the central kink of a mixed-helicity ribbon doped with S-811 performs a continuous piston-like motion. b) The device displays no sign of fatigue over ten cycles of alternating irradiation. c) A magnet connected to the kink $(\mathrm{m} \approx 2$ mg) undergoes a push-pull shuttling motion, a motion further transmitted to another magnet placed $10 \mathrm{~mm}$ below $(\mathrm{m} \approx 0.5 \mathrm{mg})$.

\subsection{Conclusions}

Inspired by biological systems, we have designed, synthesized and studied the versatile actuation modes of photoresponsive liquid-crystalline polymer springs. Light operates molecular-scale motion (cis-trans photoisomerization), which is converted into large macroscopic deformations of the springs through a mechanism that involves an increase of disorder associated with anisotropic deformations. This molecular approach gives access to (and control of ) a large diversity of morphology and photoresponses in the spiral ribbons. More than a single actuation mode is encoded inherently in these chiral objects and the actuation can be reversed when changing from one handedness to the other. Moreover, we have demonstrated that these chiral objects can be used to achieve work. These versatile 
actuators constitute a solid basis for designing novel functional materials that can provide an economic, clean and electrode-less alternative to conventional actuators.

\subsection{Acknowledgments}

We acknowledge Dr. S. P. Fletcher (University of Oxford) for the collaboration, and Mark A. Smithers (University of Twente) for assistance with TEM imaging.

\subsection{Materials and methods}

\subsubsection{General}

Monomers C6BP, C6BPN and C6M (Figure 3.10) were purchased from Synthon Chemicals. A nematic liquid crystal (E7), Chiral dopant S811 and R811 were supplied from Merck. Phenylbis(2,4,6-trimethylbenzoyl)phosphine oxide (Ciba Irgacure 819) and dichloromethane were purchased from Sigma-Aldrich. Twist cells with a $50 \mu \mathrm{m}$ cell gap were either purchased from the Electrical Heating Company or home-made using a Sunever 150 alignment layer from Nissan Chemical Industries. UV-vis spectra were recorded on a Perkin Elmer Lambda 850 UV-vis spectrophotometer. ${ }^{1} \mathrm{H}$ NMR spectra was recorded on Bruker $400 \mathrm{MHz}$ spectrometer. All SEM images were performed using Carl Zeiss Merlin microscope operating in range $0.67-1.00 \mathrm{kV}$. Samples were placed on carbon conductive tab attaching on sample mount and was analyzed without treatment.
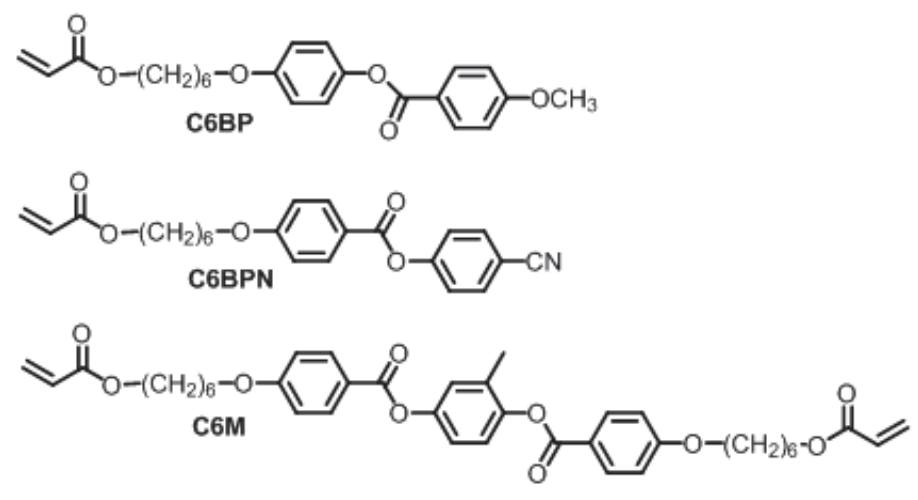

Figure 3.10 Liquid crystal monomers used in this work. C6BP and C6BPN are monoacrylates and $\mathbf{C 6} \mathbf{M}$ is a diacrylate. They are in a ratio of 3:1:2. 
Bio-inspired strategies to convert light into macroscopic twist motion

\subsubsection{Synthesis of the molecular switch}

Molecular switch 1 was synthesized according to literature procedures ${ }^{39,40}$ (Scheme 3.1) and its ${ }^{1} \mathrm{H}$ NMR spectrum was in accordance with previous reports; ${ }^{1} \mathrm{H}$ NMR $(300 \mathrm{MHz}$, $\left.\mathrm{CDCl}_{3}\right) \delta$ ppm 1.42-1.59 (m, 4H) 1.68-1.77 (m, 2H) 1.80-1.88 (m, 2H) 4.04 (t, J=6.4 Hz, 2H) $4.18(\mathrm{t}, J=6.8 \mathrm{~Hz}, 2 \mathrm{H}) 5.81(\mathrm{dd}, J=10.4,1.5 \mathrm{~Hz}, 1 \mathrm{H}) 6.12(\mathrm{dd}, J=17.2,10.4 \mathrm{~Hz}, 1 \mathrm{H})$ $6.40(\mathrm{dd}, J=17.4,1.5 \mathrm{~Hz}, 1 \mathrm{H}) 6.98(\mathrm{~d}, J=9 \mathrm{~Hz}, 2 \mathrm{H}) 7.87$ (d, $J=9 \mathrm{~Hz}, 2 \mathrm{H})$

The photoisomerization of $\mathbf{1}$ in hexane was followed by UV-visible spectroscopy (Figure $3.11)$.

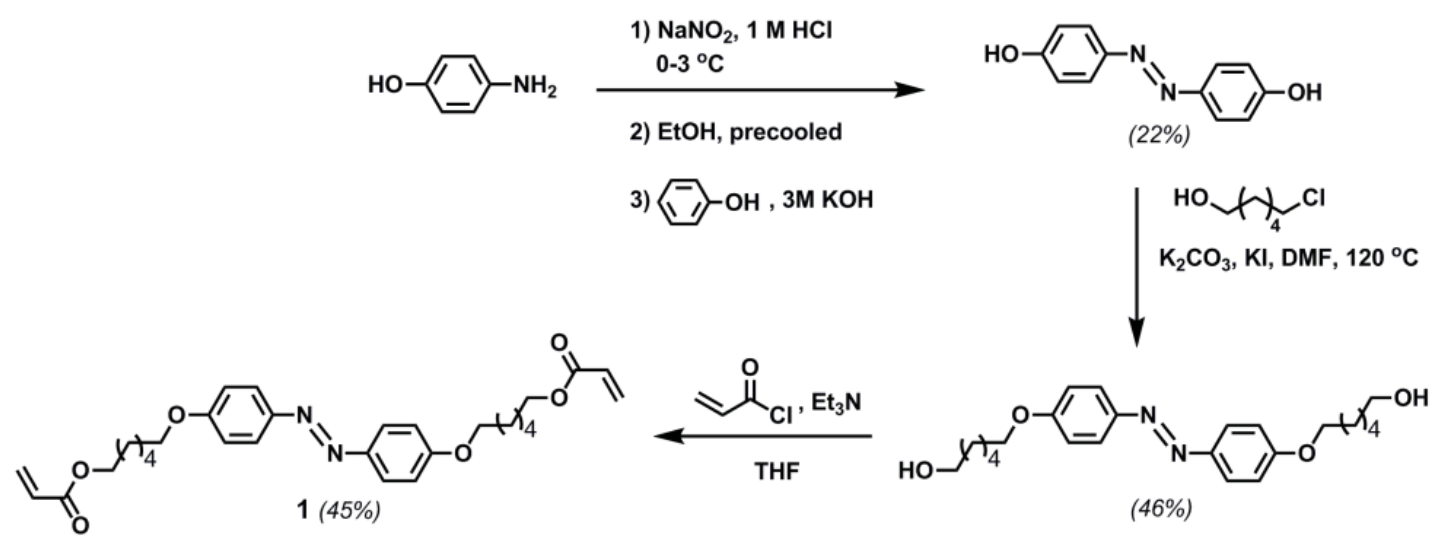

Scheme 3.1 Synthetic route for molecular switch $1 .^{39,40}$

a

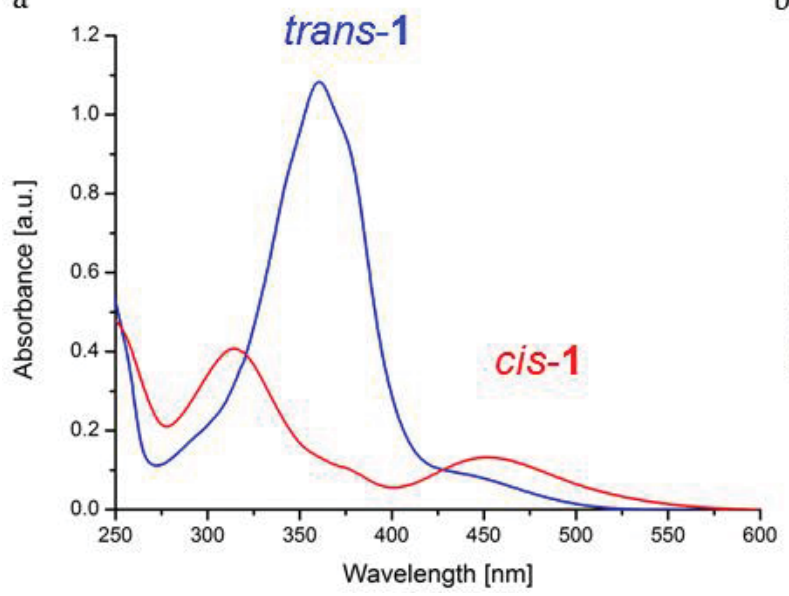

$\mathrm{b}$

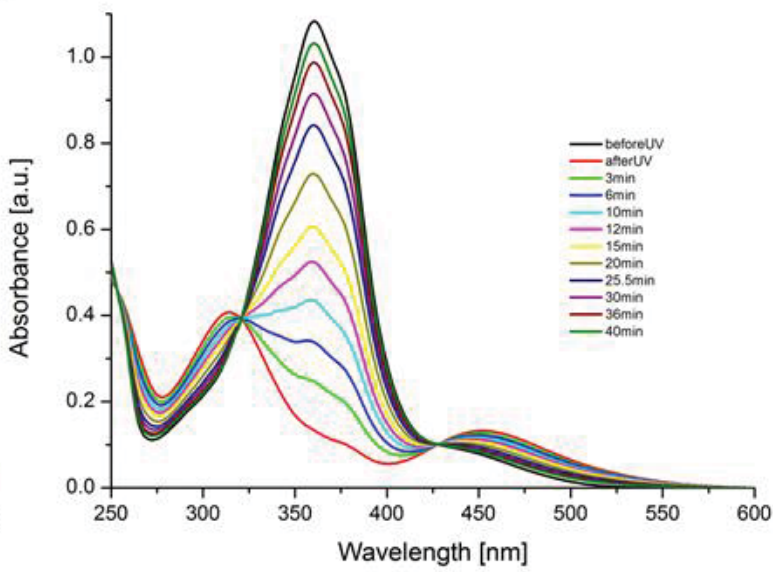

Figure 3.11 a) UV-vis spectra of azobenzene 1 in hexane b) UV-vis spectra of azobenzene 1 during thermal relaxation in ambient conditions. 


\subsubsection{Preparation of the springs, structural characterization and photo-mechanical properties}

Monomers C6BP, C6BPN and C6M were mixed in a 3:1:2 ratio by weight. A nematic liquid crystal (E7), used to prevent crystallization upon insertion in the cell, was added to the monomer mixture in a ratio of 1:2.5 by weight in favour of the monomer mixture. 1 was added in $10 \mathrm{wt} \%$ to the matrix described previously. Based on helical twisting power (HTP, Figure 3.12), chiral dopant $\mathbf{S 8 1 1}$ or $\mathbf{R 8 1 1}$ was added to the mixture $(0.04 \mathrm{wt} \%)$ to induce a cholesteric helix with a pitch of $200 \mu \mathrm{m}$. Phenylbis(2,4,6-trimethylbenzoyl)phosphine oxide (Ciba Irgacure 819) was used as a photoinitiator with a $1.0 \mathrm{wt} \%$ concentration in the cholesteric mixture. All compounds were dissolved and mixed in dichloromethane. Afterwards, the solvent was evaporated at $40^{\circ} \mathrm{C}$ under a nitrogen stream. The twist cells were preheated at $55{ }^{\circ} \mathrm{C}$ prior to their use and the photoresponsive cholesteric liquid crystal was introduced into the cell at $48{ }^{\circ} \mathrm{C}$. The cell was irradiated from the top using an Edmund MI150 high-intensity illuminator equipped with a cutoff filter $(\lambda \geq 420 \mathrm{~nm})$ to initiate the polymerization. After irradiation the cell was left in the oven at $55^{\circ} \mathrm{C}$ overnight. An illustration for this procedure is provided in Figure 3.13.

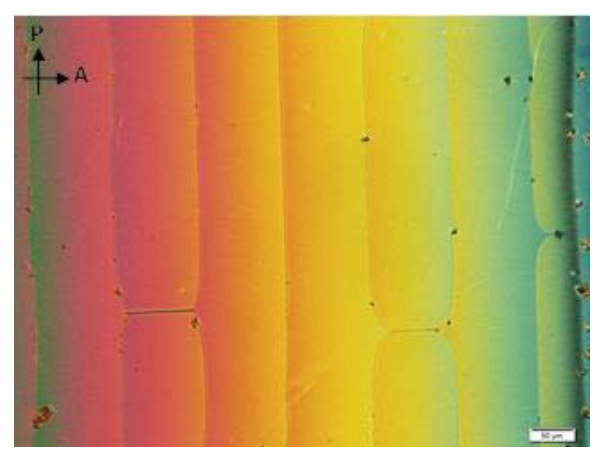

Figure 3.12 HTP determination of chiral dopant. By using Grandjean-Cano method, average distance between disclination lines $\left(\mathrm{R}_{\mathrm{av}}=103.67 \mu \mathrm{m}\right.$, scale bar $\left.=50 \mu \mathrm{m}\right)$ presented from wedge cell $(\tan \theta=0.0194)$ of polymerized liquid crystal mixture, composing of S811 $(2 \%)$, azo-1 (10\%), C6M (20.71\%), C6BP (31.07\%), C6BP (10.36\%), E7 (24.86\%) and Iragacure $819(1 \%)$ by weight, under cross-polarizer provided $4,022 \mu \mathrm{m}$ cholesteric pitch. According to equation $[\mathrm{HTP}=1 /($ c.p.ee $)], \mathrm{HTP}_{\mathrm{wt}}$ of chiral dopant $\mathbf{S 8 1 1}$ was thus determined as 12.43 $\mu \mathrm{m}^{-1}$. 

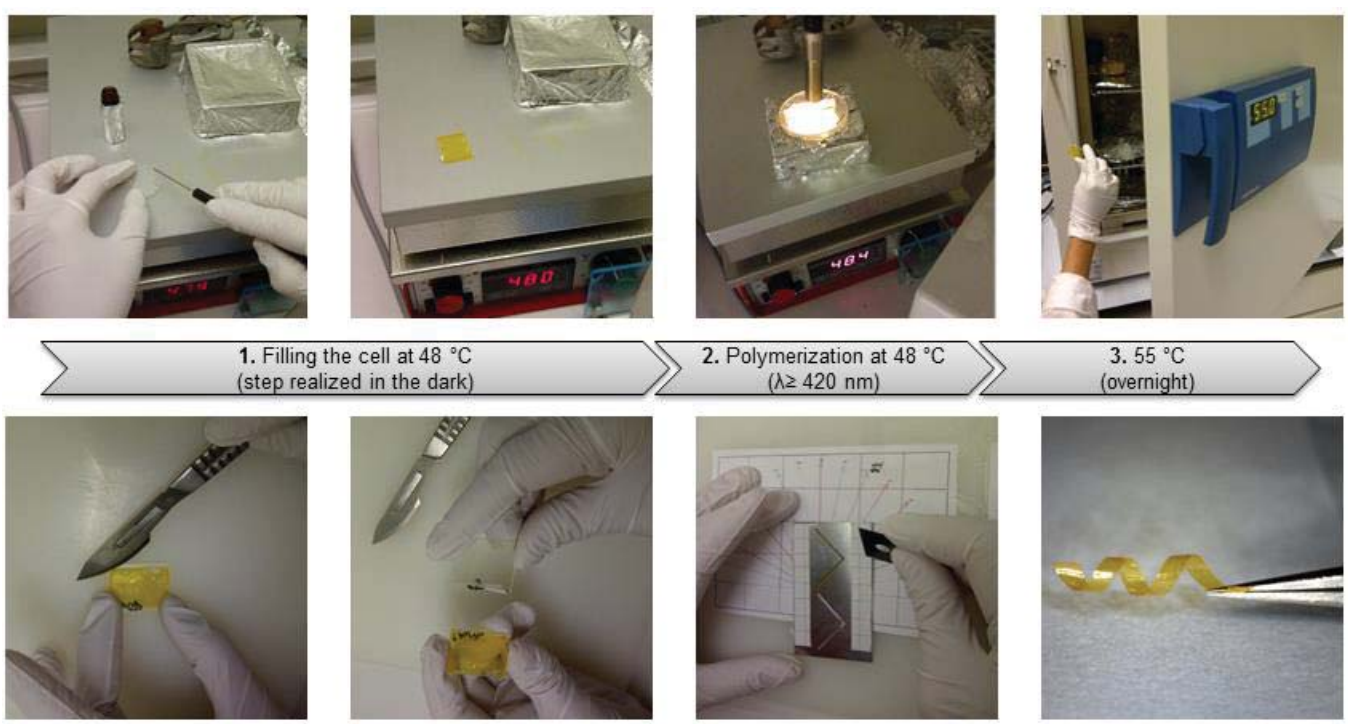

2. Polymerization at $48^{\circ} \mathrm{C}$

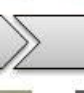

(overnight)
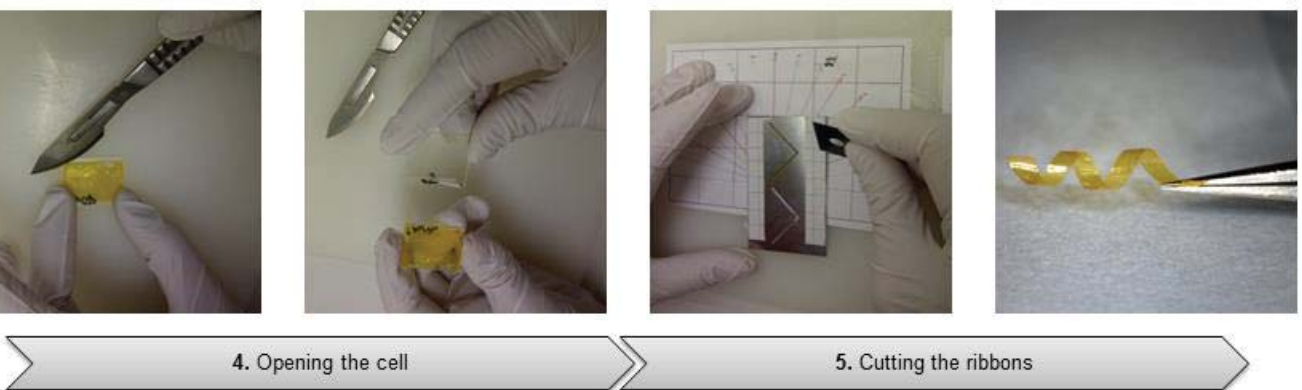

5. Cutting the ribbons

Figure 3.13 Illustration of the fabrication process.

The twist cells were frozen with liquid nitrogen and then opened using a scalpel to reveal the polymer film. The film was cut in different directions to provide ribbons with a variety of shapes. After cutting, the ribbons were dried at $55^{\circ} \mathrm{C}$ for two hours. The width of the ribbons was adjusted to $\sim 0.8 \mathrm{~mm}$. The length (L) of the one-handed ribbons was $\sim 0.8-1.3$ $\mathrm{cm}$ and the length of the mixed-helicity ribbons was $\sim 4 \mathrm{~cm}$. The thicknesses of the ribbons and the molecular organization through the film were evaluated by using scanning electron microscopy (Figure 3.14). The thicknesses were $43 \mu \mathrm{m}$ for films prepared in commercial cells and $48 \mu \mathrm{m}$ for those prepared in home-made cells, in good agreement with the $50 \mu \mathrm{m}$ gap of the cells that were used.
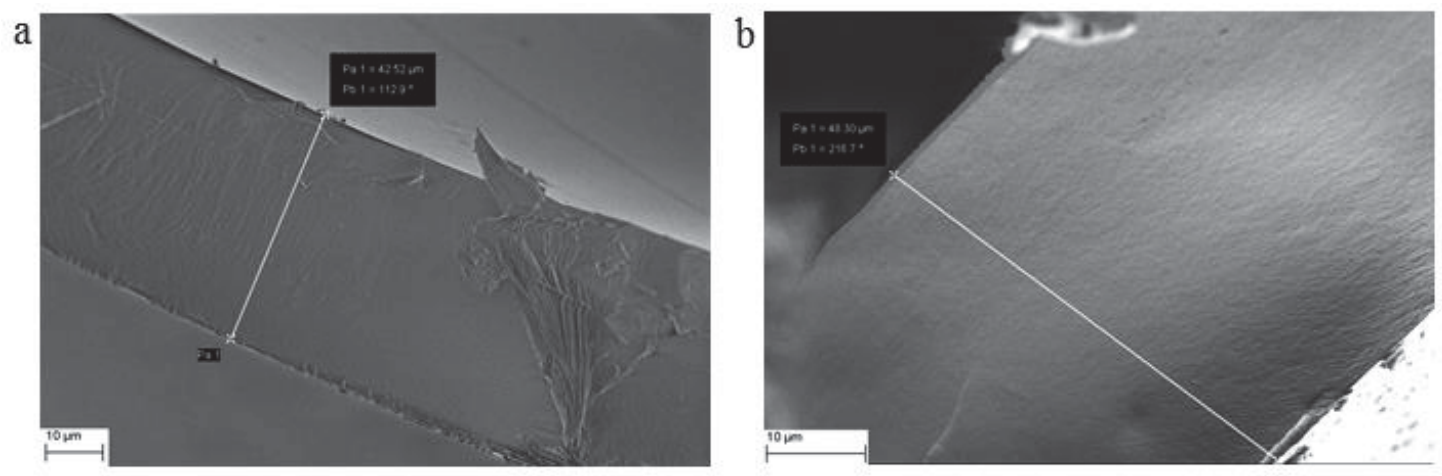

Figure 3.14 SEM images of ribbons prepared in a) commercial cell and b) home-made cell. scale bar $10 \mu \mathrm{m}$. 
Thermal gravimetric analysis experiments showed that the final ribbon contained $\sim 25 \%$ E7 in mass, which suggests all the material that comprised the photoresponsive liquid crystalline mixture was still present in the final ribbons (Figure 3.15). A Hönle blue point light-emitting diode (LED) lamp was used to analyse the photoresponses. Therefore, the polymer ribbon was placed $4 \mathrm{~cm}$ below one LED head and irradiated with an intensity that ranged from $30 \mathrm{~mW} \mathrm{~cm}^{-2}$ to $60 \mathrm{~mW} \mathrm{~cm}^{-2}$. The light-driven deformations were followed using a Dino-Lite Pro AM4113T USB microscope with $\times 29$ magnification.

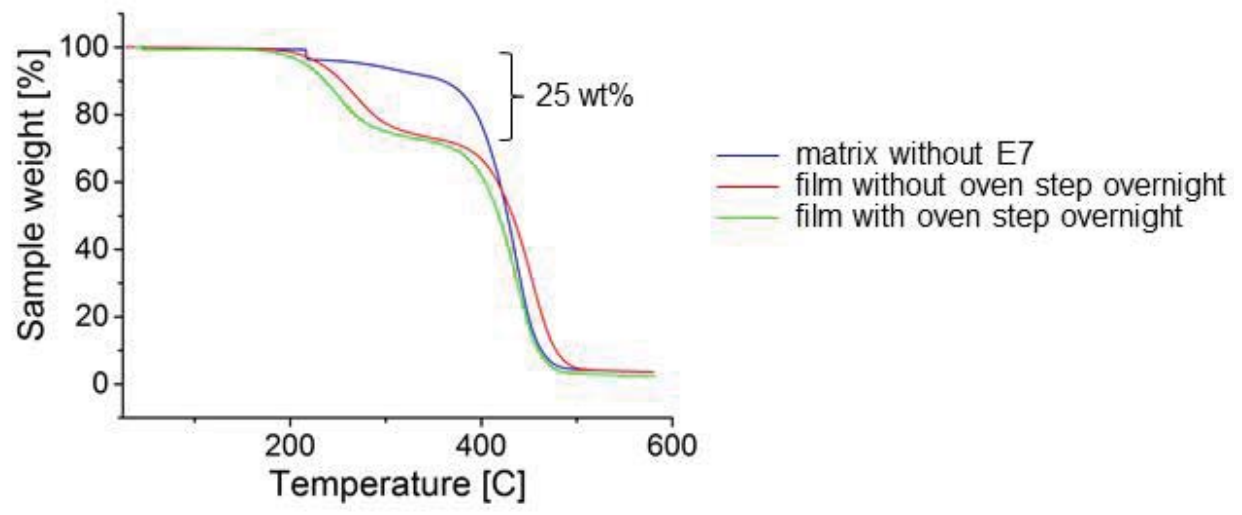

Figure 3.15 Thermogravimetric analysis of the ribbons. 
Bio-inspired strategies to convert light into macroscopic twist motion

3.5.4 Supplementary information; additional shape, responsive behaviors and characterization of the ribbons
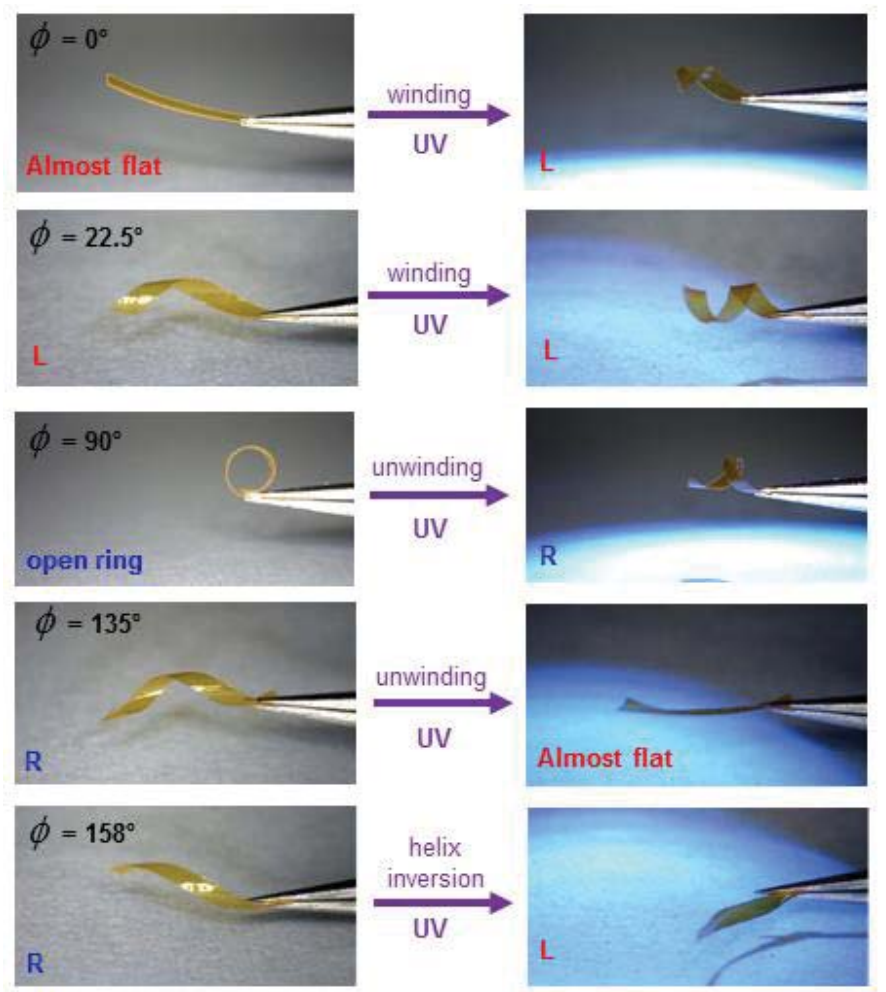

Figure 3.16 Additional images for the shape selection and the photoresponse of ribbons doped with S811. 

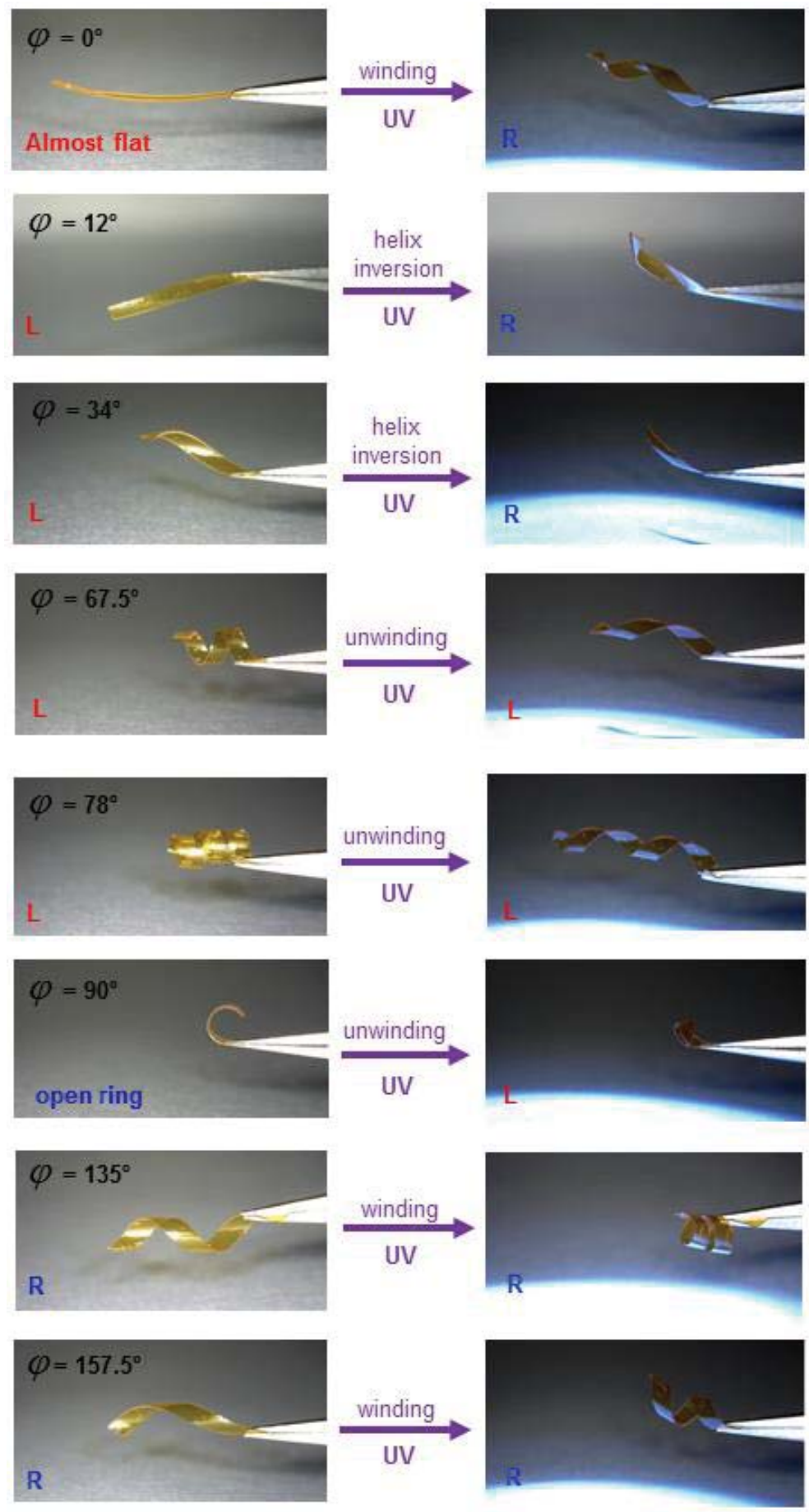

Figure 3.17 Additional images for the photoresponse of $\mathbf{R 8 1 1}$ ribbons. 
Bio-inspired strategies to convert light into macroscopic twist motion
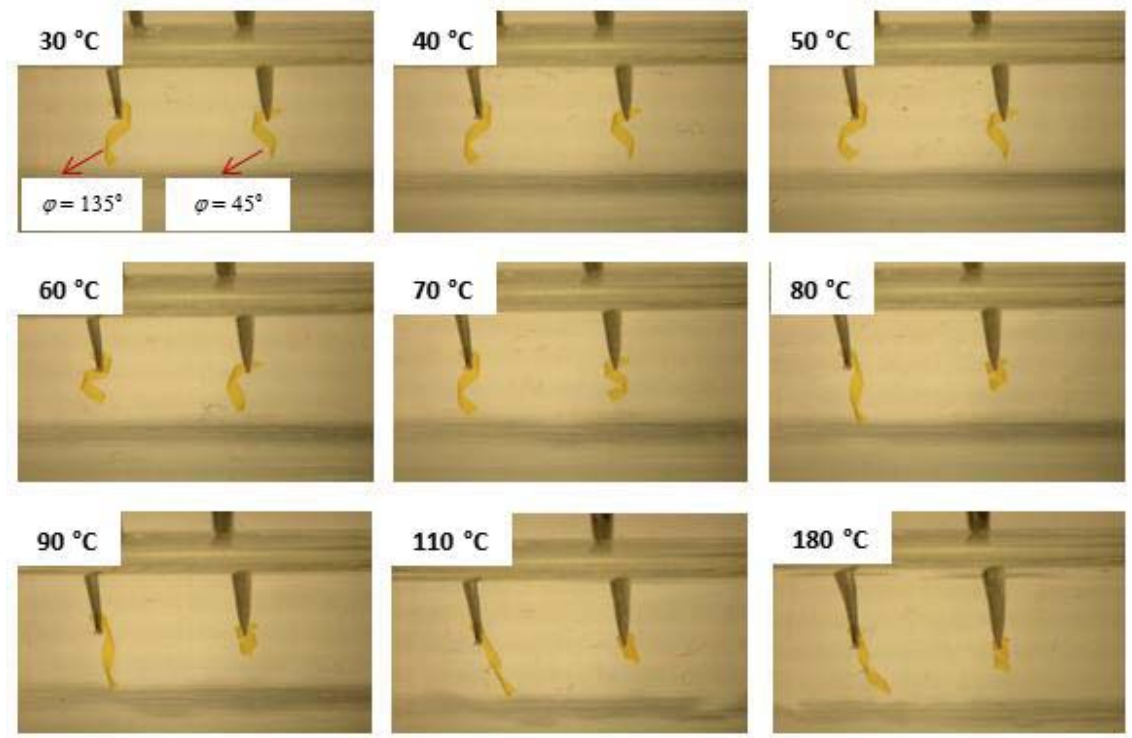

Figure 3.18 Thermoresponsive behavior of the ribbons doped with $\mathbf{R 8 1 1}$.
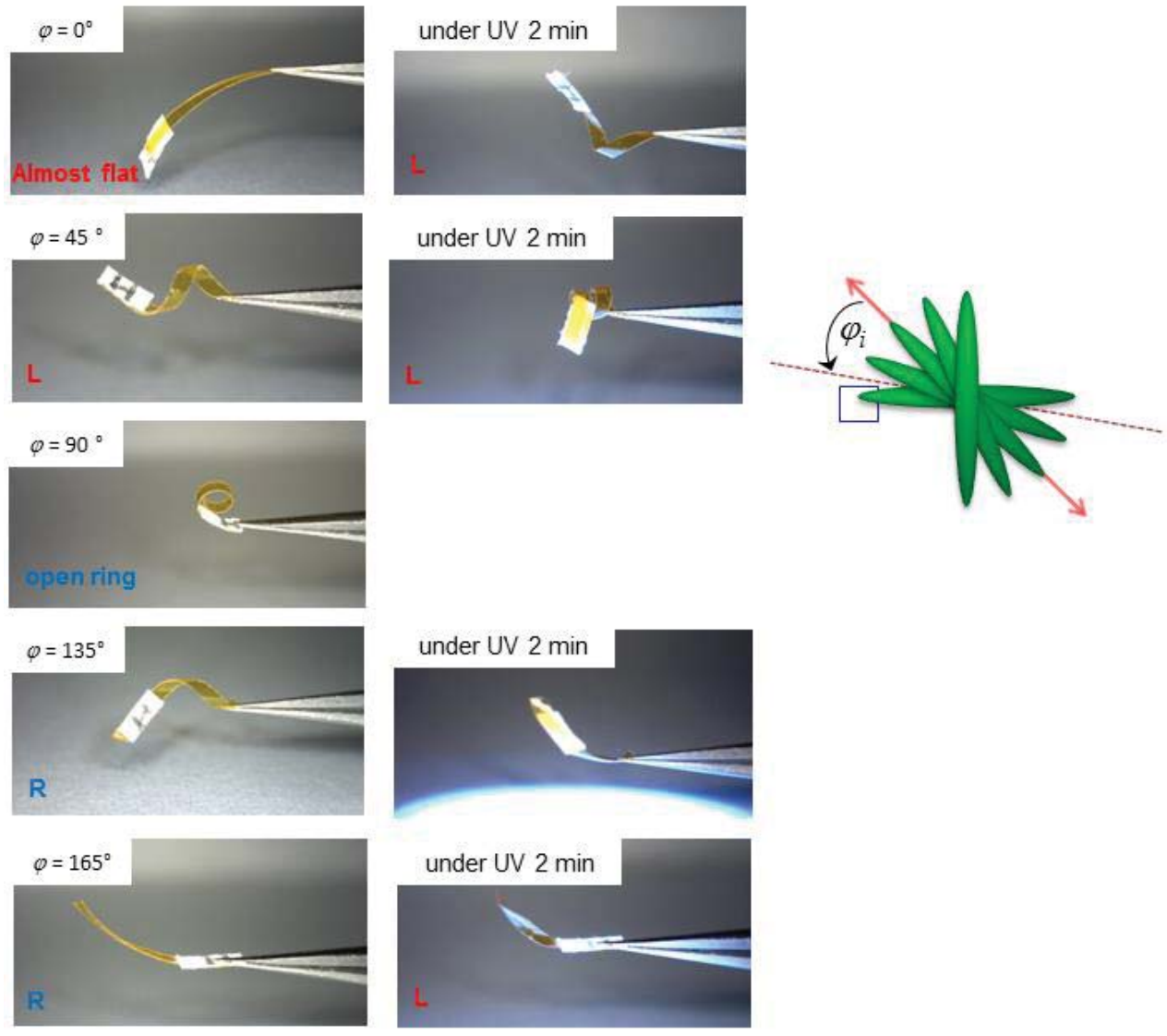

Figure 3.19 Marking the face that curls on ribbons doped with S811. The sticker marks the bottom part of the film, and ends up inside the ribbon. 

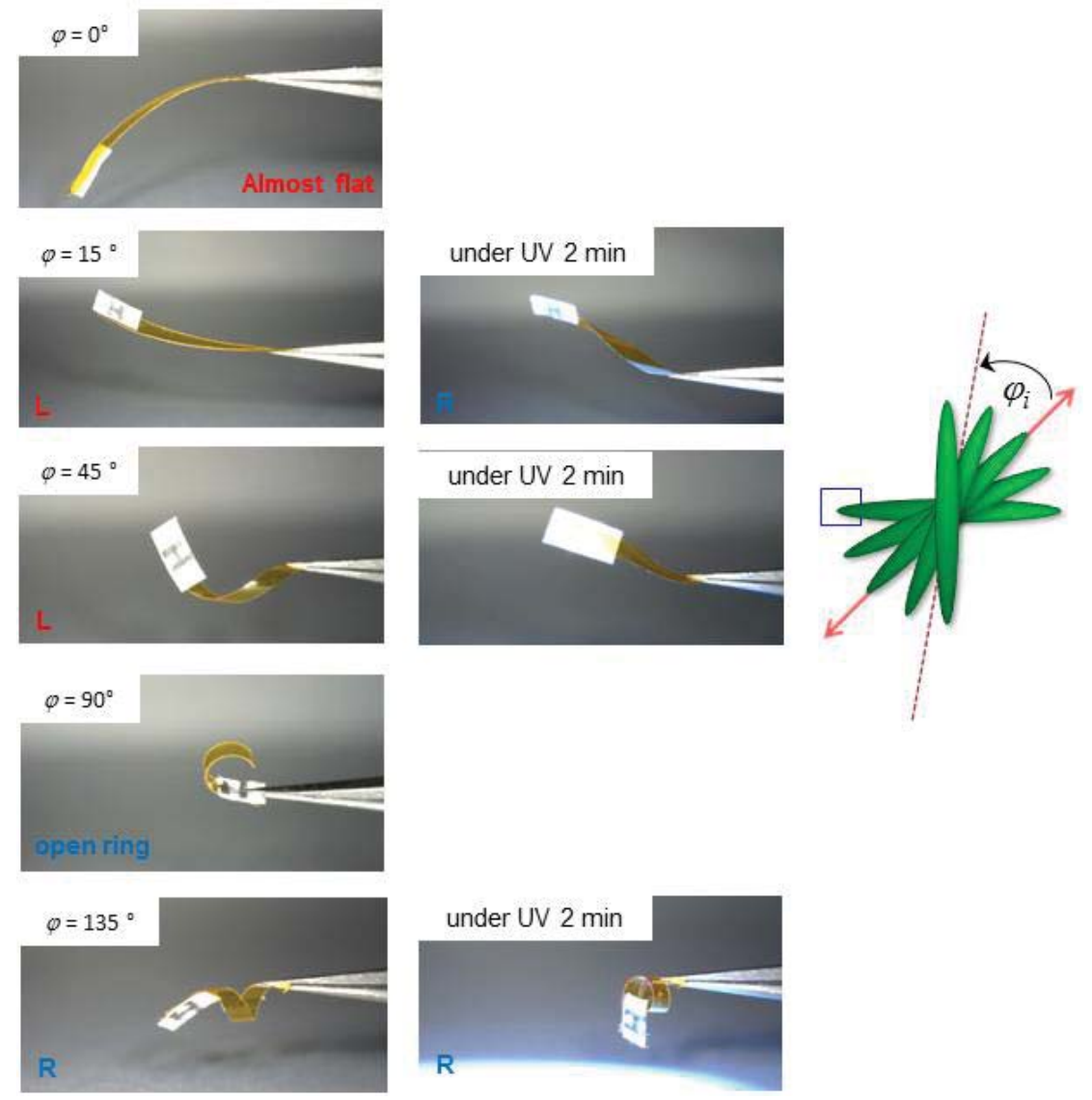

Figure 3.20 Marking the face that curls on ribbons doped with R811. The sticker marks the bottom face of the film, which ends up inside the curled ribbon.
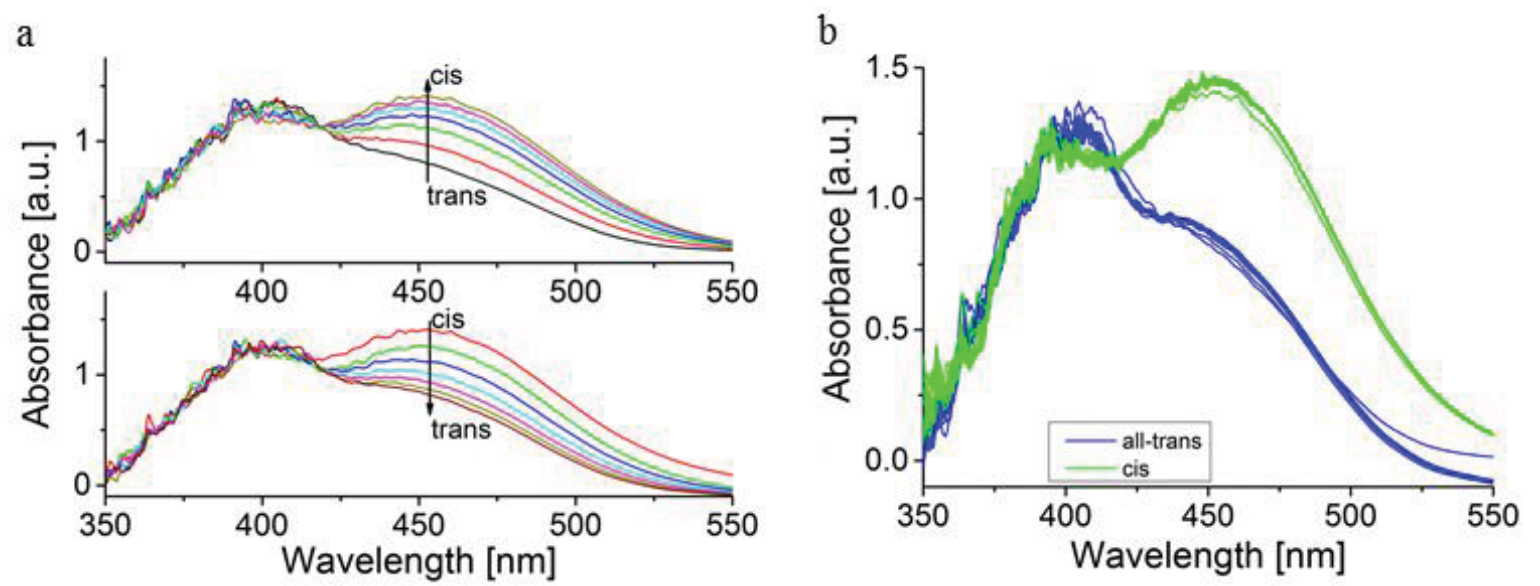

Figure 3.21 UV-vis spectra of the polymerized film in $50 \mathrm{~mm}$ thick twist cell. a) Change of signal over time during 1 min of UV irradiation [LED lamp 20\% intensity] (top) when transform isomerizes to cis-form and during $1 \mathrm{~min}$ of visible irradiation [halogen lamp without filter] (bottom) when cis-form isomerizes back to trans-form. b) Alternating irradiation of the film with UV-light and visible light (10 cycles) shows always same signal. 
Bio-inspired strategies to convert light into macroscopic twist motion

Calculation work produced by ribbon (Figure 3.7) relative to the work of a diarylethene cocrystal $^{15}$

Our ribbon (Figure 3.7);

Mass of magnet $\left(\mathrm{m}_{1}\right)=7.07 \times 10^{-6} \mathrm{~kg}$, Gravitational acceleration $(\mathrm{g})=9,80 \mathrm{~m} / \mathrm{s}^{2}$, Distance $(\mathrm{d})$ $=0,0078 \mathrm{~m}$. and Time $(\mathrm{t})=90 \mathrm{~s}$

$$
\begin{array}{ll}
\text { Work }(\mathrm{W}): & \mathrm{W}=\mathrm{m} . \mathrm{g} \cdot \mathrm{d} \\
& \mathrm{W}_{\text {ribbon }}=\left(7 \cdot 07 \times 10^{-6}\right) \cdot(9,80) \cdot(0,0078)=5,42 \times 10^{-7} \mathrm{~J} \\
\text { Power }(\mathrm{P}): & \mathrm{P}=\mathrm{W} / \mathrm{t} \\
& \mathrm{P}_{\text {ribbon }}=\left(5,42 \times 10^{-7}\right) / 90=6,02 \times 10^{-9} \mathrm{~W} \cdot=6 \mathrm{nW}
\end{array}
$$

Mass of ribbon $\left(\mathrm{m}_{2}\right)=9.3 \times 10^{-7} \mathrm{~kg}$, Mass of $\mathbf{1}\left(\mathrm{m}_{3}\right)=9.3 \times 10^{-8} \mathrm{~kg}$, Molecular weight of $\mathbf{1}$ $\left(\mathrm{Mw}_{1}\right)=522.63$ and mole of switch $1\left(\mathrm{~mol}_{\text {switch }}\right)=1.78 \times 10^{-7} \mathrm{~mol}$

Work relative to mole of switch $1\left(\mathrm{~W}_{\text {rel.mol 1 }}\right): \mathrm{W}_{\text {rel., mol } 1}=\mathrm{W}_{\text {ribbon }} / \mathrm{mol}_{\text {switch1 }}$

$$
\mathrm{W}_{\text {rel.mol 1 }}=5,42 \times 10^{-7} / 1.78 \times 10^{-7}=3,04 \mathrm{~J} / \mathrm{mol}
$$

\section{Diarylethene co-crystal ${ }^{15}$}

Co-crystal contain photo-switching unit (diarylethene derivative (io) and perfluoronaphthalene (fn) in mole ratio 1:2.

Mass of co-crystal $\left(\mathrm{m}_{\text {co-crystal }}\right)=0,17 \times 10^{-3} \mathrm{~kg}$, Molecular weight of io $\left(\mathrm{Mw}_{\mathrm{io}}\right)=620,67$, Molecular weight of $\mathrm{fn}\left(\mathrm{Mw}_{\mathrm{fn}}\right)=272,09$

$$
\begin{aligned}
& \mathrm{mol}_{\mathrm{io}}=?: \quad \quad \mathrm{m}_{\mathrm{co}-\mathrm{crystal}}=\mathrm{mol}_{\mathrm{io}} \cdot \mathrm{Mw}_{\mathrm{io}}+\mathrm{mol}_{\mathrm{fn}} \cdot \mathrm{Mw}_{\mathrm{fn}} \\
& =\mathrm{mol}_{\mathrm{io}} \cdot \mathrm{Mw}_{\mathrm{io}}+2 \mathrm{~mol}_{\mathrm{io}} \cdot \mathrm{Mw}_{\mathrm{fn}} \\
& 0,17 \times 10^{-3}=(620,67) \cdot \mathrm{mol}_{\text {io }}+(2) \cdot(272,09) \cdot \mathrm{mol}_{\text {io }} \\
& \mathrm{mol}_{\mathrm{io}}=1,46 \times 10^{-7} \mathrm{~mol} \text {. }
\end{aligned}
$$

Reported work of co-crystal $=0,43 \mu \mathrm{J}$

Work relative to mole of switch io $\left(\mathrm{W}_{\text {rel.mol io }}\right): \mathrm{W}_{\text {rel., mol io }}=\mathrm{W}_{\text {co-crystal }} / \mathrm{mol}_{\text {switch io }}$

$$
\begin{aligned}
\mathrm{W}_{\text {rel., mol io }}=\mathrm{W}_{\text {co-crystal }} / \mathrm{mol}_{\text {switch io }} & =\left(0,43 \times 10^{-6}\right) / 1,46 \times 10^{-7} \\
& =2.95 \mathrm{~J} / \mathrm{mol}
\end{aligned}
$$

\subsection{References}

1 Feynman, R. P. in Miniaturization; (ed. Gilbert, H. D.), Reinhold, 1961.

2 Drexler, K. E. Nanosystems: Molecular Machinery, Manufacturing and 
Computation, Wiley, 1992.

3 Koumura, N.; Zijlstra, R. W. J.; van Delden, R. A.; Harada, N.; Feringa, B. L. Nature 1999, 401, 152.

4 Leigh, D. A.; Wong, J. K. Y.; Dehez, F.; Zerbetto, F. Nature 2003, 424, 174.

$5 \quad$ Fletcher, S. P.; Dumur, F.; Pollard, M. M.; Feringa, B. L. Science 2005, 310, 80.

6 Balzani, V.; Credi, A.; Raymo, F. M.; Stoddart, J. F. Angew. Chem. Int. Ed. 2000, $39,3348$.

7 von Delius, M.; Geertsema, E. M.; Leigh, D. A. Nat. Chem. 2010, 2, 96.

$8 \quad$ Muraoka, T.; Kinbara, K.; Aida, T. Nature 2006, 440, 512.

9 Kudernac, T.; Ruangsupapichat, N.; Parschau, M.; Macia, B.; Katsonis, N.; Harutyunyan, S. R.; Ernst, K.-H.; Feringa, B. L. Nature 2011, 479, 208.

10 Lewandowski, B.; De Bo, G.; Ward, J. W.; Papmeyer, M.; Kuschel, S.; Aldegunde, M. J.; Gramlich, P. M. E.; Heckmann, D.; Goldup, S. M.; D’Souza, D. M.; Fernandes, A. E.; Leigh, D. A. Science 2013, 339, 189.

11 Berna, J.; Leigh, D. A.; Lubomska, M.; Mendoza, S. M.; Perez, E. M.; Rudolf, P.; Teobaldi, G.; Zerbetto, F. Nat. Mater. 2005, 4, 704.

12 Eelkema, R.; Pollard, M. M.; Vicario, J.; Katsonis, N.; Ramon, B. S.; Bastiaansen, C. W. M.; Broer, D. J.; Feringa, B. L. Nature 2006, 440, 163.

13 Yamada, M.; Kondo, M.; Mamiya, J.-i.; Yu, Y.; Kinoshita, M.; Barrett, C. J.; Ikeda, T. Angew. Chem. Int. Ed. 2008, 47, 4986.

14 Juluri, B. K.; Kumar, A. S.; Liu, Y.; Ye, T.; Yang, Y.-W.; Flood, A. H.; Fang, L.; Stoddart, J. F.; Weiss, P. S.; Huang, T. J. ACS Nano 2009, 3, 291.

15 Morimoto, M.; Irie, M. J. Am. Chem. Soc. 2010, 132, 14172.

16 Browne, W. R.; Feringa, B. L. Nat. Nanotechnol. 2006, 1, 25.

17 Ariga, K.; Mori, T.; Hill, J. P. Adv. Mater. 2012, 24, 158.

18 Coskun, A.; Banaszak, M.; Astumian, R. D.; Stoddart, J. F.; Grzybowski, B. A. Chem. Soc. Rev. 2012, 41, 19.

19 Spinks, G. M. Angew. Chem. Int. Ed. 2012, 51, 2285.

20 Schliwa, M. Molecular Motors; Wiley, 2003.

21 Mahadevan, L.; Matsudaira, P. Science 2000, 288, 95.

22 Armon, S. Science 2011, 333, 1726. 
Bio-inspired strategies to convert light into macroscopic twist motion

23 Gerbode, S. J.; Puzey, J. R.; McCormick, A. G.; Mahadevan, L. Science 2012, 337, 1087.

24 Zhu, L.; Al-Kaysi, R. O.; Bardeen, C. J. J. Am. Chem. Soc. 2011, 133, 12569.

25 Kitagawa, D.; Nishi, H.; Kobatake, S. Angew. Chem. Int. Ed. 2013, 52, 9320.

26 Lee, K. M.; Smith, M. L.; Koerner, H.; Tabiryan, N.; Vaia, R. A.; Bunning, T. J.; White, T. J. Adv. Funct. Mater. 2011, 21, 2913.

27 Warner, M.; Terentjev, E. M. Liquid Crystal Elastomers Oxford Univ. Press, 2003.

28 Natansohn, A.; Rochon, P. Chem. Rev. 2002, 102, 4139.

29 Yu, Y.; Nakano, M.; Ikeda, T. Nature 2003, 425, 145.

30 van Oosten, C. L.; Bastiaansen, C. W. M.; Broer, D. J. Nat. Mater. 2009, 8, 677.

31 van Oosten, C. L.; Harris, K. D.; Bastiaansen, C. W. M.; Broer, D. J. Eur. Phys. J. E 2007, 23, 329.

32 Sawa, Y.; Ye, F.; Urayama, K.; Takigawa, T.; Gimenez-Pinto, V.; Selinger, R. L. B.; Selinger, J. V. Proc. Natl. Acad. Sci. 2011, 108, 6364.

33 Sawa, Y. e. a. Phys. Rev. E 2013, 88.

34 Teresi, L.; Varano, V. Soft Matter 2013, 9, 3081.

35 Forterre, Y.; Dumais, J. Science 2011, 333, 1715.

36 van Oosten, C. L.; Corbett, D.; Davies, D.; Warner, M.; Bastiaansen, C. W. M.; Broer, D. J. Macromolecules 2008, 41, 8592.

37 Harris, K. D.; Cuypers, R.; Scheibe, P.; van Oosten, C. L.; Bastiaansen, C. W. M.; Lub, J.; Broer, D. J. J. Mater. Chem. 2005, 15, 5043.

38 Hosono, N.; Kajitani, T.; Fukushima, T.; Ito, K.; Sasaki, S.; Takata, M.; Aida, T. Science 2010, 330, 808.

39 Mossety-Leszczak, B.; Wlodarska, M.; Galina, H.; Bak, G. W. Mol. Cryst. Liq. Cryst. 2008, 490, 52.

40 Li, C.; Lo, C.-W.; Zhu, D.; Li, C.; Liu, Y.; Jiang, H. Macromol. Rapid Commun. 2009, 30, 1928. 


\section{Chapter 4}

\section{Preserving the delayed relaxation of $o$-fluorinated azobenzenes in liquid crystal polymer networks}

Liquid crystal polymer networks incorporating azobenzenes are promising materials for soft robotics, primarily because of the anisotropic shape changes they undergo under irradiation with light. However, in most cases, these materials go back to their initial shape once illumination stops, which can be a limitation for applications to robotics, morphing and bio-medication, where stability of the activated shape is required on a longer time scale. Previous attempts to exploit the intrinsic properties of molecular photo-switches in order to stabilize the photo-activated liquid crystal polymer networks were unsuccessful, primarily because of lack of amplification mechanisms that would preserve the switching specificity. In this chapter, we design liquid crystal polymer networks incorporating an o-fluoroazobenzene as a photoswitch. By incorporating the switch as a cross-linking unit and by avoiding $\mathrm{H}$ bonding, we succeed in delaying the relaxation of the polymer network substantially, with a half-life time of at least eight days. 
Preserving the delayed relaxation of $o$-fluorinated azobenzenes in liquid crystal polymer networks

\subsection{Introduction}

Soft responsive materials are capable of displaying sophisticated motion and shape deformation, and hence they have demonstrated potential to perform complex mechanical work, in analogy with biological materials. ${ }^{1-4}$ Engineering new materials and actuators for soft robotics has consequently become a major contemporary thrust. ${ }^{5-9}$ Liquid crystal polymer networks are prominent candidates towards the design of such a soft machinery, ${ }^{10-13}$ as they share some of the elastic properties of conventional polymers with the orientational order and anisotropic properties of liquid crystals. ${ }^{10,14}$ Also, the orientation of liquid crystal polymer networks can be directed by using e.g. surface treatment and external stimuli, a property that facilitates the design of a variety of macroscopic architectures and functions. ${ }^{14-16}$ Besides, a broad range of stimuli can be used to activate shape changes effectively in liquid crystal polymer networks. ${ }^{17-19}$ Arguably, a promising approach to modulate the shape of liquid crystal polymers networks is to make use of light-driven molecular switches, the motion of which is amplified, from the molecular scale up to the functional, macroscopic level. ${ }^{20-22}$ This strategy can be implemented by embedding molecular switches as co-monomers in the liquid crystal polymer networks. As mentioned in previous chapters also, azobenzene-based switches ${ }^{23-25}$ are the main photo-switches that are used to photo-activate liquid crystal networks. Currently, it is commonly accepted that the switching of azobenzenes is amplified because the order parameter of the system is reduced when the cis-isomer is formed. ${ }^{26}$ Recent results have suggested an alternative mechanism, upon observing that the mechanical deformation of liquid crystal polymer networks is enhanced by simultaneous irradiation with UV and visible light, because it promotes a cistrans oscillation of the azobenzenes. ${ }^{27}$ The reverse reaction that leads to shape recovery after irradiation has ceased, remains less investigated and challenging to control. Control over the relaxation of photo-active liquid crystal networks would facilitate applications where the photo-driven formation of a stable shape is needed, for example for robotic grippers, ${ }^{28,29}$ shape morphing, ${ }^{15,20}$ aerospacial applications ${ }^{30}$ and surgical devices. ${ }^{31}$

Regarding the formation of stable shapes, photo-responsive liquid crystal polymer networks are underperforming compared to classical polymer networks, mainly because as soon as irradiation stops, the decay of the cis-form drives the relaxation of the liquid crystal 
polymer network towards its original state. ${ }^{32}$ In contrast, many classical cross-linked polymers display shape memory properties, i.e. they retain their activated shape temporarily even after removal of the external stimulus. ${ }^{33}$ However, achieving the shape deformation of these cross-linked polymers typically requires heating them above a transition temperature (either the melting- or the glass transition- temperature) and a subsequent thermal quenching freezes the mobility of network and consequently stabilizes the shape. In practice however, using high temperatures can be a drawback. A few works have consequently attempted to control the rate of shape recovery of liquid crystal polymer networks. Finkelmann and Velasco ${ }^{34}$ have examined the rate of shape recovery of liquid crystal networks containing methoxy-substituted azobenzenes, with different spacer lengths, although the research aimed at accelerating the shape reversibility by promoting fast relaxation of the switches. Another study reported by White et al. ${ }^{35}$ has demonstrated shape changes of a liquid crystal network containing $m$-fluorinated azobenzenes, under irradiation with either UV or visible light. However, the slow relaxation of the fluorinated switch was lost at the macroscopic level, likely because the switch was introduced as a pendant group (Figure 4.1b) instead of being a cross-linker (Figure 4.1a).

Beyond soft robotics, the relaxation kinetics of substituted azobenzenes has been investigated in other materials also. Elyashevitch ${ }^{36}$ and coworkers have succeeded in retaining an optical pattern on a polystyrene/liquid crystal composite by controlling the relaxation of $o$-methyl substituted azobenzenes. Besides, o-chloroazobenzenes have been grafted to a biological receptor, where the thermal stability of the cis-isomer was harnessed for the opening of ion channels. ${ }^{37}$ These examples suggest that the molecular properties of azobenzenes can be amplified at the macroscopic level, including their dynamic properties. However, this is not always the case, and there were also some examples where relaxation at the macroscale seemed de-correlated from the intrinsic properties of the dopants. For example, as above-mentioned, some designs of liquid crystal polymer networks incorporating $m$-fluoroazobenzenes exhibit nearly identical opto-mechanical properties than their classical counterparts. ${ }^{35}$ In another investigation, the deformation of single crystals of perfluorinated azobenzene has revealed completely irreversible. ${ }^{38}$

Herein, we achieve control over the rate of shape reversion in light-responsive liquid crystal polymer networks by implementing strategies in which the intrinsic properties of 
Preserving the delayed relaxation of $o$-fluorinated azobenzenes in liquid crystal polymer networks

azobenzenes are preserved. We opted for $o$-fluoroazobenzene derivatives, that were first reported by Hecht and coworkers ${ }^{39}$ as potential candidates because the cis-isomer typically displays a half-life time that reaches up to two years, in solution. Besides, the switch maintains structural planarity, ${ }^{39}$ which is suitable for orientational control over the adjacent liquid crystal moieties in the network. We investigate the relaxation rate of the $O$ fluoroazobenzene switch, both as a monomer and once embedded as a cross-linker in a liquid crystal polymer network that is devoid of H-bonding. In the resulting material, shape reversal is at least four times slower than its counterpart doped with a classical (non-fluorinated) azobenzene.

a
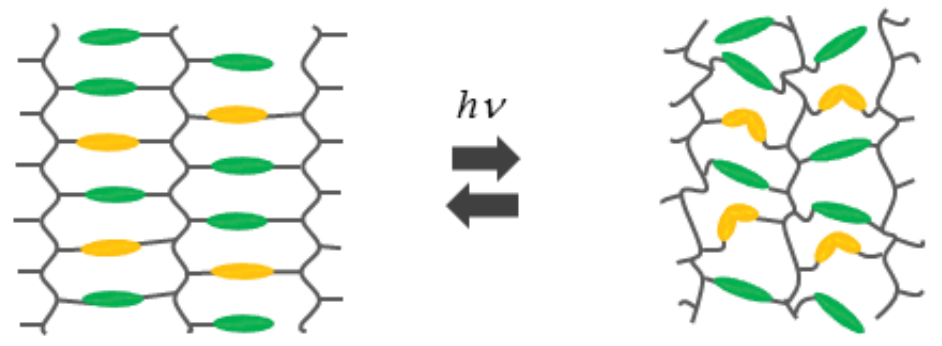

$\mathrm{b}$
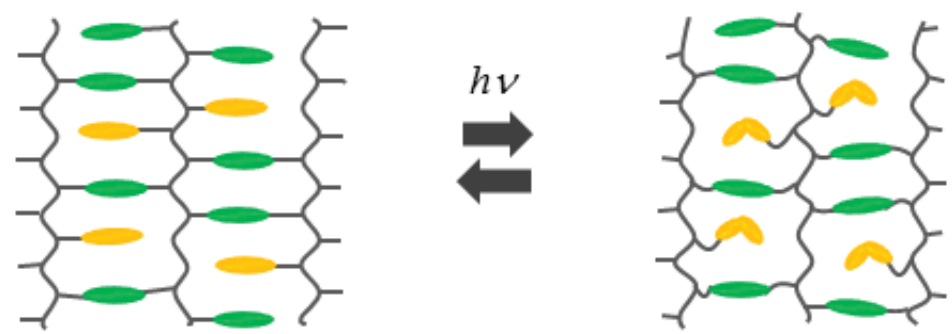

Figure 4.1 Photo-induced mechanical deformation and relaxation of liquid crystals polymer networks containing derivative azobenzene switch monomer as a) cross-linker and b) pendant group. Adapted from [27]. 


\subsection{Results and discussion}

Gaining insight into the mechanisms by which the molecular properties of switches allow control over shape recovery, requires comparing the isomerization and relaxation of $o^{-}$ fluorinated azobenzenes and those of conventional azobenzenes, both in an isotropic solvent and once embedded in the liquid crystal polymer network.

\subsubsection{Photochemistry of F-1 in solution}

UV-vis spectroscopy (Fig. 4.2b) and ${ }^{1} \mathrm{H}-\mathrm{NMR}$ (Fig. 4.2c) showed that upon irradiation with UV light ( $\lambda=365 \mathrm{~nm}$ ) F-1 switches (Fig. 4.2a) from trans- to cis-isomer with high photoconversion (91\% of cis-form at PSS), when dissolved in dichloromethane. The thermal stability of cis-F-1 was examined by ${ }^{1} \mathrm{H}-\mathrm{NMR}$, by monitoring the cis to trans backisomerization in the dark (Fig 4.2c). Five days after irradiation with UV light, the spectra demonstrate that approximately $80 \%$ of the cis-F-1 in solution was still present. Meanwhile, cis-H-1 was undetectable within about a day after irradiation, as revealed by UV-vis spectroscopy (section 4.5.4, Fig 4.8). In solution, the considerably longer lifetime of cis-F-1 compared to its conventional counterpart H-1 was accounted by stabilization via inductive effect of $o$-fluorine as previous report. ${ }^{39} \mathrm{We}$ attribute the decrease in the life time of cis-F-1 (half-life $\sim 49$ days, Figure 4.2d) compared to its individual core structure (half-life $\sim 2$ years $)^{39}$ to the electronic donating properties of the alkoxy moiety in the spacer, which are likely to mitigate the effect of $o$-fluorine in stabilizing the cis-isomer. 
Preserving the delayed relaxation of $o$-fluorinated azobenzenes in liquid crystal polymer networks

a

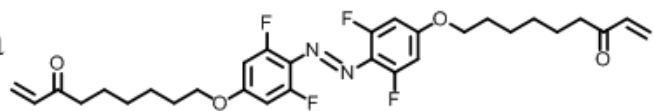

F-1

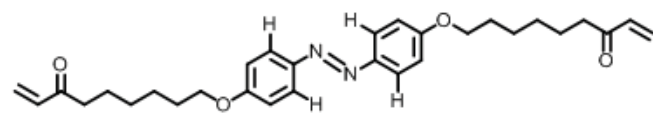

H-1

$\mathrm{C}$

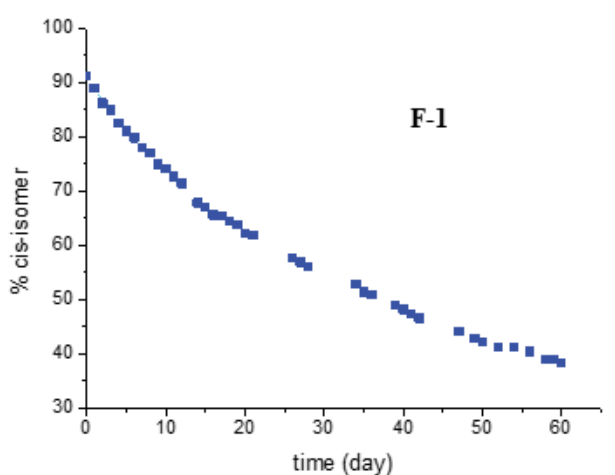

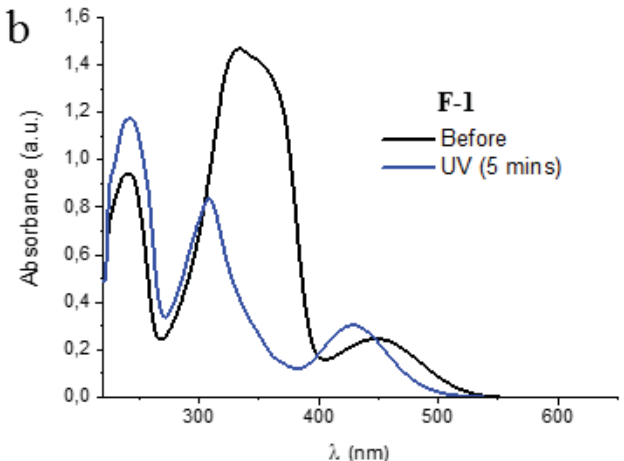

d

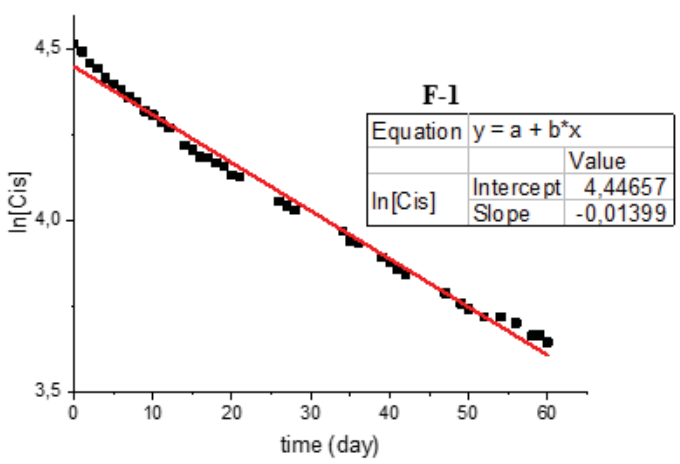

Figure 4.2 a) Molecular structure of the $o$-fluorinated switchable monomer F-1 used in this study, and its classical counterpart H-1. The photochemistry of F-1 is followed in an isotropic solvent (DCM, $10 \mathrm{~min}$ irradiation, $77 \mathrm{~mW} / \mathrm{cm}^{2}$ ). b) UV-vis absorption of $10^{-4} \mathrm{M}$ of F-1 in DCM upon irradiation with UV light $(\lambda=365 \mathrm{~nm})$. c) Corresponding cis-trans back isomerization of F-1 in the dark as investigated by ${ }^{1} \mathrm{H}-\mathrm{NMR}$ (see also section 4.5.4) d) First order kinetics plot of cis-trans back isomerization under dark condition, provides a slope $\mathrm{b}^{*}$ $=0.014$ and indicates a half-life time $\left(\mathrm{t}_{1 / 2}=0,693 / \mathrm{b}^{*}\right)$ of $\sim 49$ days.

\subsubsection{Photochemistry of F-1 once covalently bound to the liquid crystal polymer network}

The photo-isomerization and thermal relaxation of F-1 and H-1 once embedded covalently in the network were studied by UV-vis absorption spectroscopy (Figure 4.3a and b). Comparing their thermal-relaxation in the dark revealed that the thermal relaxation of cisF-1 was slowed down significantly compared to its conventional counterpart. After two days, the characteristic absorption band of cis-F-1 was still present (Figure 4.3a), whereas with same period, H-1 embedded in the network showed nearly full conversion of the cis-isomer into the trans-isomer (Figure 4.3b). Plotting the absorption decay of the cis-isomers against time highlighted the lower decreasing rate of cis-F-1 compared to $\mathbf{H - 1}$, over the same period 
(Figure 4.3c). Thereby, the relative speed of cis- to trans-relaxation of F-1 and $\mathbf{H}-\mathbf{1}$ presented the same trend whether in solution or as part of the network. In other words, the slow thermal relaxation of F-1 is maintained in the polymer system. Interestingly, the preservation of F-1 in the liquid crystal network was not observed in earlier studies where the relaxation rate of a $m$-fluoroazobenzene was comparable to its conventional counterpart, once it was covalently bound to the liquid crystal network. ${ }^{35}$ Beyond the relaxation properties only, White et al. report on nearly identical photomechanical and optical properties between the fluorinated liquid crystal polymer network and its classical counterpart. The fact that molecular properties of the switches are not expressed at the macroscale was attributed by the authors to a degradation in their switching properties, that would arise from i) hydrogen bonding between the $m$-fluoroazobenzene switches incorporated as pendant groups, that hampers their isomerization and favours the trans form over the cis form and ii) the presence of polydomains in the liquid crystal polymer network, that implies a random orientation of the switches in the network, a situation that could further inhibit the isomerization and reorientation of the switches. ${ }^{35}$ Based on this report we designed a liquid crystal polymer network where these potential limiting factors are attenuated or avoided, by 1) locating the switch in the main polymer chain 2) by diluting the switch within the network and 3) by promoting a well-defined alignment at the interface, instead of a degenerate planar alignment with random orientation of the molecules in plane. Importantly, incorporating F-1 in the polymer backbone amplifies the actuation of the network upon isomerization. ${ }^{34}$ 
Preserving the delayed relaxation of $o$-fluorinated azobenzenes in liquid crystal polymer networks
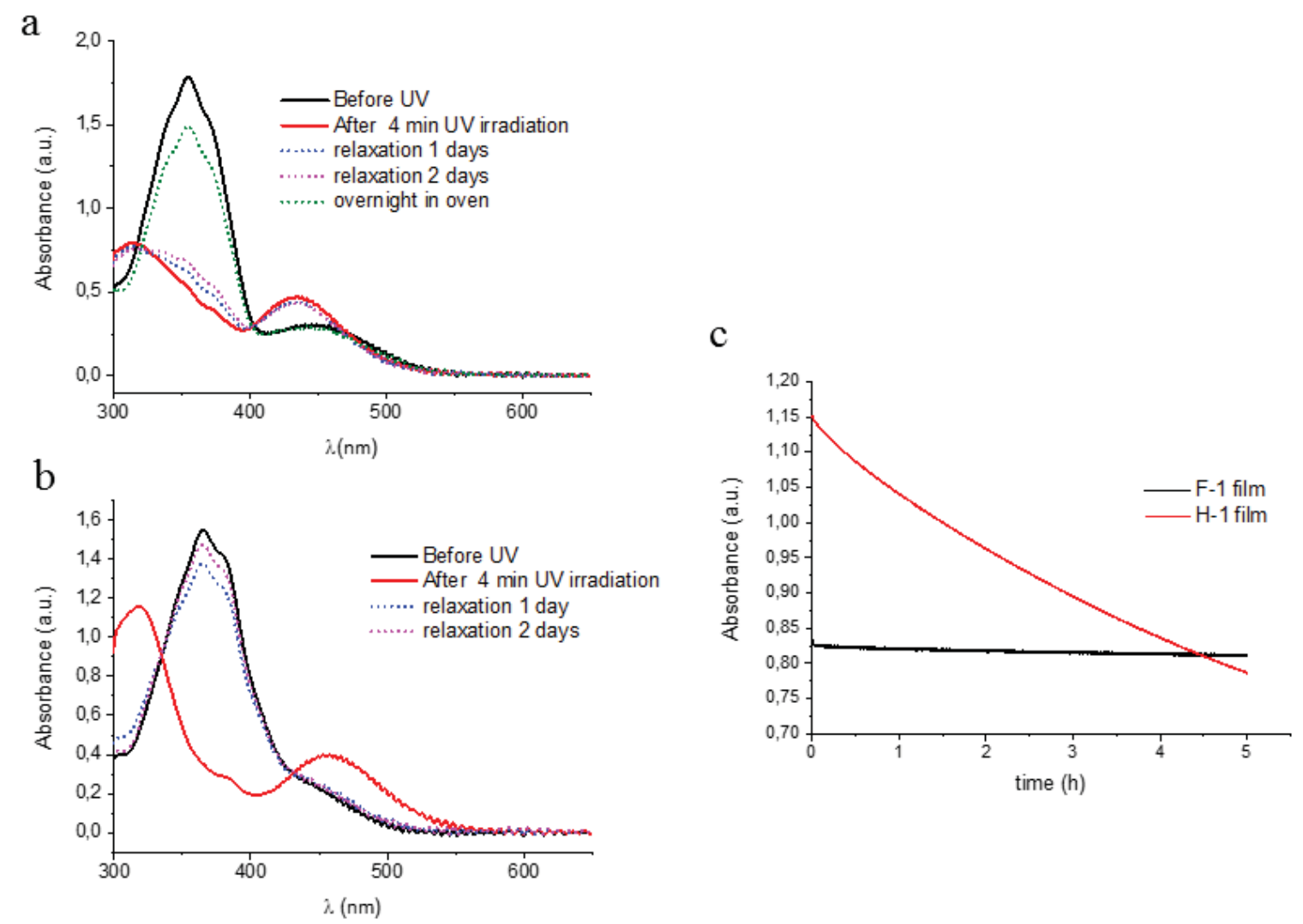

Figure 4.3 Change in UV-vis absorption spectra after 2 min irradiation with UV light (170 $\mathrm{mW} / \mathrm{cm}^{2}$ ) and during thermal cis-trans isomerization in the dark, for liquid crystal polymer networks containing a) F-1 and b) H-1. c) Time dependence of cis-trans isomerization in the dark for liquid crystal polymer films containing $\mathbf{F - 1}$ and $\mathbf{H}-\mathbf{1}$, where the wavelength is set at the maximum absorption wavelength of each switch, $\lambda=311 \mathrm{~nm}$ and $\lambda=317 \mathrm{~nm}$ respectively.

\subsubsection{Photo-actuation and shape relaxation}

The shape recovery of the films incorporating either $\mathbf{H}-\mathbf{1}$ or $\mathbf{F}-\mathbf{1}$ was inspected in the dark (Figure 4.4). Our aim was to study the shape recovery of films having an identical geometry, and based on our recent work on liquid crystal polymer springs (see Chapter 3$)^{23}$ we decided to investigate the photo-actuation and shape relaxation of ribbons. Therefore, we cut ribbons out of the thin films of liquid crystal network. The cutting direction is measured by the angle $\varphi$. For $\varphi=90^{\circ}$ (Figure 4.4), regardless of whether a spiral ribbon or a helicoid is formed (Figure 4.4b(ii) and c(ii)), it was clear that F-1 displayed a slower rate of shape relaxation compared to the H-1 based film; specifically, after two days the $\mathbf{H - 1}$ based film was nearly half way back towards its original shape (Figure $4.4 \mathrm{c}(v)$ ) while the film containing $\mathbf{F}-\mathbf{1}$ 
showed only minor shape changes (Figure $4.4 \mathrm{~b}(v)$ ). Hence, the film incorporating F-1 exhibited greater performance in terms of shape retention.

\section{a}

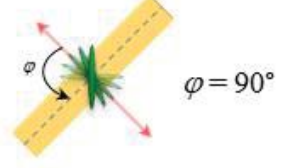

$\mathrm{b}$
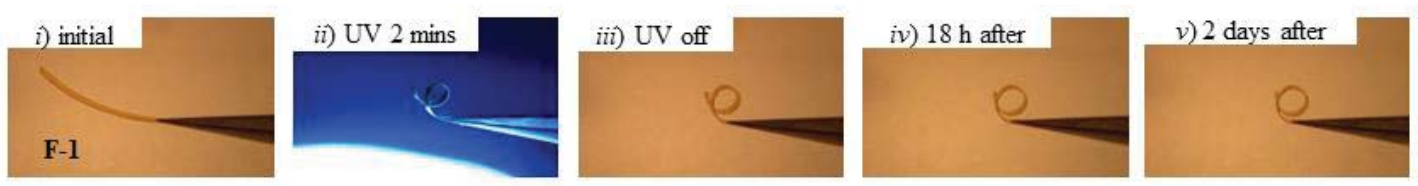

$\mathrm{c}$
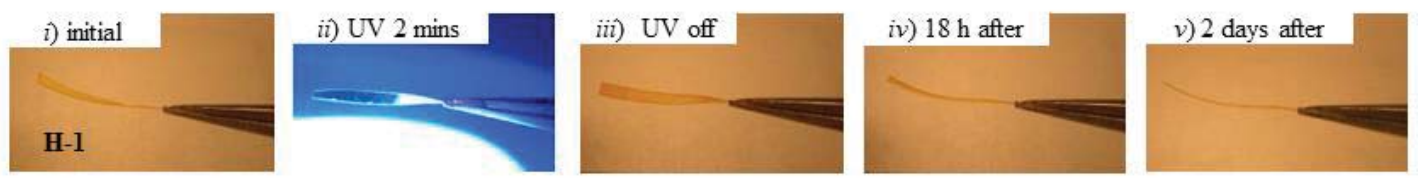

Figure 4.4 a) Schematic illustration for the molecular organization in a ribbon cut at $\varphi=90^{\circ}$. UV-response and shape relaxation in the dark for ribbons having identical geometries $(\varphi=$ $90^{\circ}$ ), made out of liquid crystal polymer networks containing b) F-1 and c) $\mathbf{H}-\mathbf{1}$ (half-life = $\sim 2$ days); 2 min irradiation with $170 \mathrm{~mW} / \mathrm{cm}^{2}$ light was used for both experiments.

Other ribbons with different shapes were selected from our library of photo-responsive springs (Figure 4.5), and their shape relaxation rates were compared, in the dark (Figure 4.6). Within 8 days after irradiation with UV light, a ribbon made from $\mathbf{H}-1\left(\varphi=0^{\circ}\right)$ moved back to its original shape. In contrast, an F-1 ribbon that transforms into a similar shape ( $\varphi$ $=112.5^{\circ}$ ), displays a delay in its shape relaxation (Figure 4.6). We finally note that the ribbon cut out from the F-1 film continues bending, albeit in a moderate manner, after irradiation with UV-light has stopped (Figure 4.6(iii-x)-b). We anticipate that this behavior results from the re-orientation and re-organization of the polymer in the presence of cis-F-1, which takes place gradually. 
Preserving the delayed relaxation of $o$-fluorinated azobenzenes in liquid crystal polymer networks

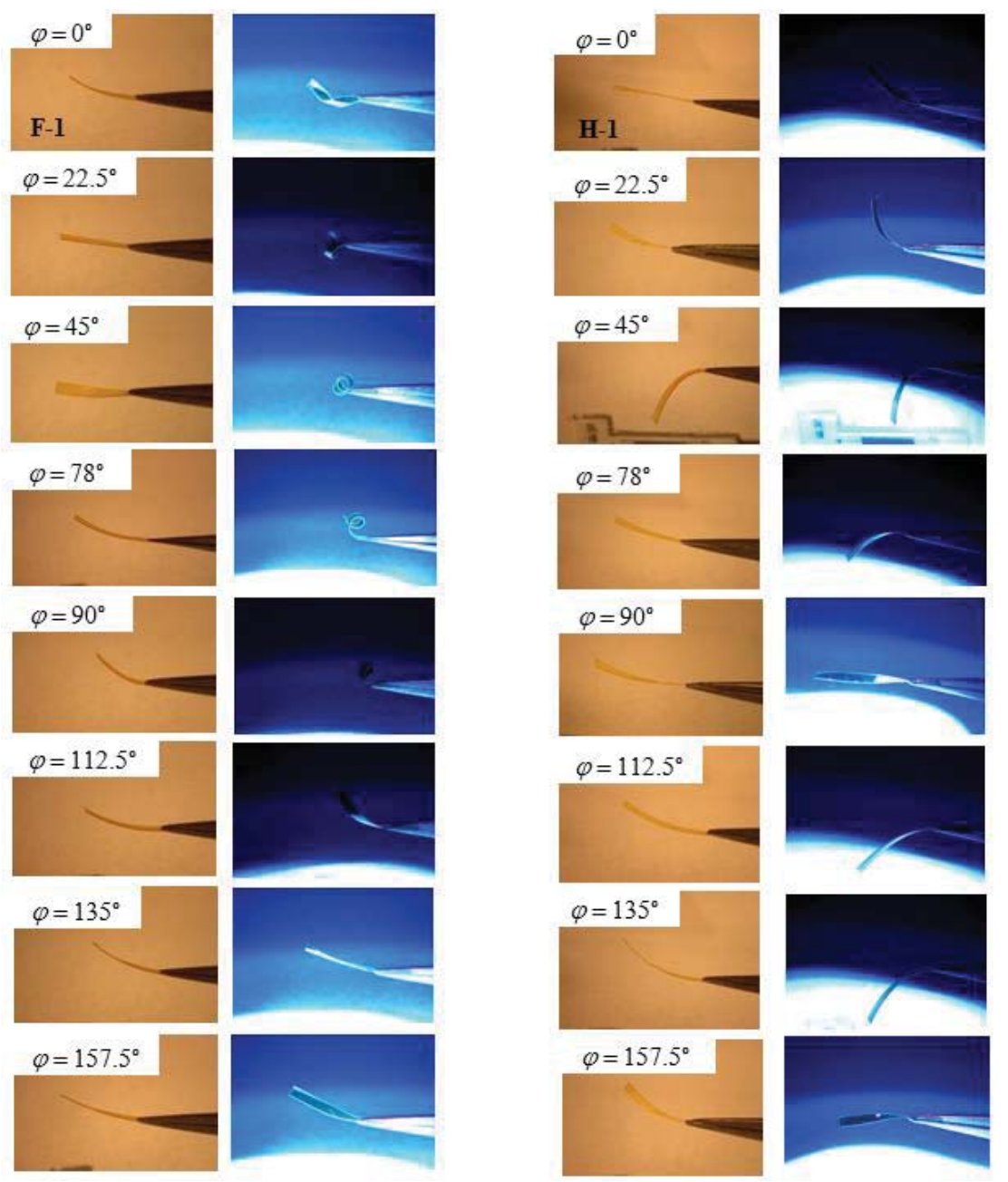

Figure 4.5 UV-response of liquid crystal film with F-1 (two columns at left) and with $\mathbf{H}-\mathbf{1}$ (the two columns on the right). Conditions of irradiation at $\lambda=365 \mathrm{~nm}: 2 \mathrm{~min}$ at $170 \mathrm{~mW} / \mathrm{cm}^{2}$

Comparing the dynamic behavior of $\mathbf{H - 1}$ and F-1 once incorporated in the network (Figure 4.3a and $\mathrm{b}$ ) and the shape relaxation that is observed at the macroscopic level (Figure 4.4 and 4.6), it appears that the slower decay of cis-F-1 translates into a slower shape relaxation for the polymer network also. Moreover it appears that the liquid crystal polymer film (Figure $4.4 \mathrm{c}(\mathrm{v})$ and $4.6(\mathrm{v})$-a) relaxes slower than the molecular switches that are incorporated in it (Figure $4.3 \mathrm{~b}$ at $\sim 2$ days). In other words, a delay is observed in the response of the network, which we attribute to its elastic properties. Overall, we conclude that while the elasticity of the network likely affects the delayed shape relaxation of F-1 films, the main driving force lies in the intrinsic properties of the molecular switches. 

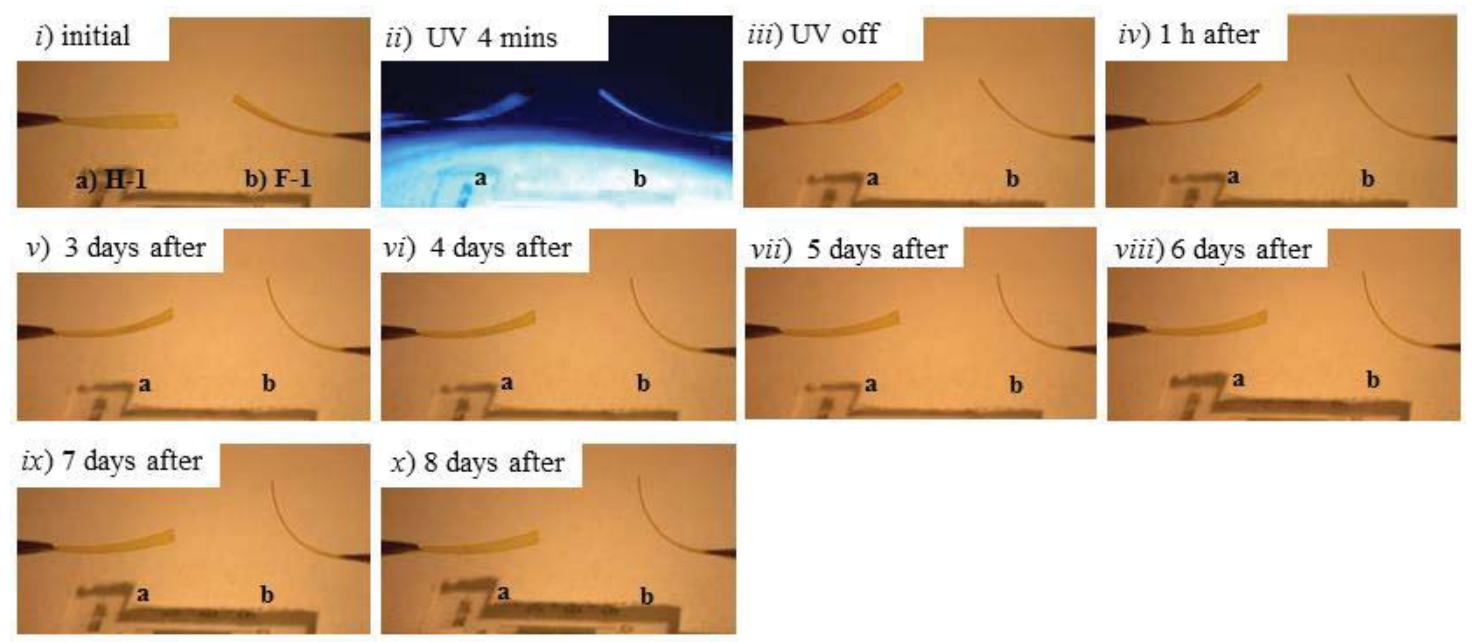

Figure 4.6 UV-response and shape relaxation of ribbons containing a) $\mathbf{H}-\mathbf{1}\left(\varphi=0^{\circ}\right)$ and b) F-1 $\left(\varphi=112.5^{\circ}\right)$.

Unlike other systems incorporating fluorinated azobenzenes as dynamic units, in particular co-crystals ${ }^{38}$ and liquid crystal networks, ${ }^{35}$ where inherent molecular properties of the switches were damaged or not preserved at macroscale, here we show that the fingerprint of molecular relaxation of F-1 is preserved in a liquid crystal polymer network (Fig. 4.3-4.4 and 4.6).

\subsection{Conclusions}

In conclusion, $\boldsymbol{o}$-fluoroazobenzene $\mathbf{F - 1}$ was designed and synthesized based on a strategy adapted from earlier reports. ${ }^{39} \mathbf{F}-\mathbf{1}$ and its conventional counterpart $\mathbf{H}-\mathbf{1}$ were incorporated covalently in liquid crystal polymer networks as switchable cross-linkers. These dynamic units performed well in transducing light into a mechanical function, e.g. bending and twisting (Figure 4.5). Analyzing the reversible switching of F-1 both as a free monomer in solution and as a linker in the liquid crystal network disclosed that its delayed relaxation is preserved in the liquid crystal polymer network. Moreover, the relaxation of the network incorporating F-1 showed a delayed relaxation compared to the networks incorporating $\mathbf{H}-\mathbf{1}$, which means that complex shapes could be formed that do not disappear when irradiation stops. Our work also provides some additional insight into the relaxation of azobenzenebased liquid crystal polymer networks and further demonstrates that the local environment of molecular switches remains key to conceal, attenuate or preserve molecular features when designing soft machines by transduction of light into mechanical work. 
Preserving the delayed relaxation of $o$-fluorinated azobenzenes in liquid crystal polymer networks

\subsection{Acknowledgments}

We acknowledge Dr. E. Anger, our collaborator within the research group of Prof. S.P. Fletcher (University of Oxford, UK) for the synthesis and structural characterization of F-1.

\subsection{Materials and methods}

\subsubsection{General}

Liquid crystal di-acrylate $(\mathrm{C} 6 \mathrm{M})$ and mono-acrylates (C6BP and $\mathrm{C} 6 \mathrm{BPN})$ were purchased from Synthon Chemicals. Chiral dopant S811 and Irgacure 819 were supplied by Sigma-Aldrich. The chemical structures of these compounds are shown in Chapter 3 (Figure $3.1 \mathrm{~b}$ and 3.10). Dichloromethane with HPLC grade for film preparation was purchased from company. Liquid crystal cells were purchased from E.H.C. Japan. The structure of F-1 was confirmed by ${ }^{1} \mathrm{H},{ }^{13} \mathrm{C}-\mathrm{NMR}$ spectroscopy and mass spectrometry. ${ }^{1} \mathrm{H}$ and ${ }^{13} \mathrm{C}$ spectra were recorded on Bruker Ascend 400. Irradiation with light was performed by using a Hönle blue point light-emitting diode lamp $\left(77-170 \mathrm{~mW} / \mathrm{cm}^{2}\right)$. UV-visible absorption spectra of $\mathbf{F - 1}$ and H-1 were measured using a Perkin Elmer Lambda 850 spectrophotometer. The photostationary state and rates of thermal isomerization were evaluated based on the integrals of the signals for the cis- and trans-isomer observed by ${ }^{1} \mathrm{H}-\mathrm{NMR}$ (Figure 4.7). The distribution of the director in the film was confirmed by polarizing optical microscopy. Shape modifications were followed using a Dino-Lite Pro AM4113T USB microscope.

\subsubsection{Synthesis of F-1}

The synthesis of F-1 is schematically summarized (Scheme 4.1). Its core structure is identical to previously reported structures, ${ }^{39}$ and was extended with long spacer to produce ample actuation in the polymer matrix as well as two acrylate arms to locate it on polymer backbone, which also contribute to larger actuation upon photo-isomerization relative to case of side chain monomer. ${ }^{34}$ The conventional azobenzene H-1 was prepared following a previously described procedure (see also chapter 3 , section 3.5 .2 ). ${ }^{40,41}$ 

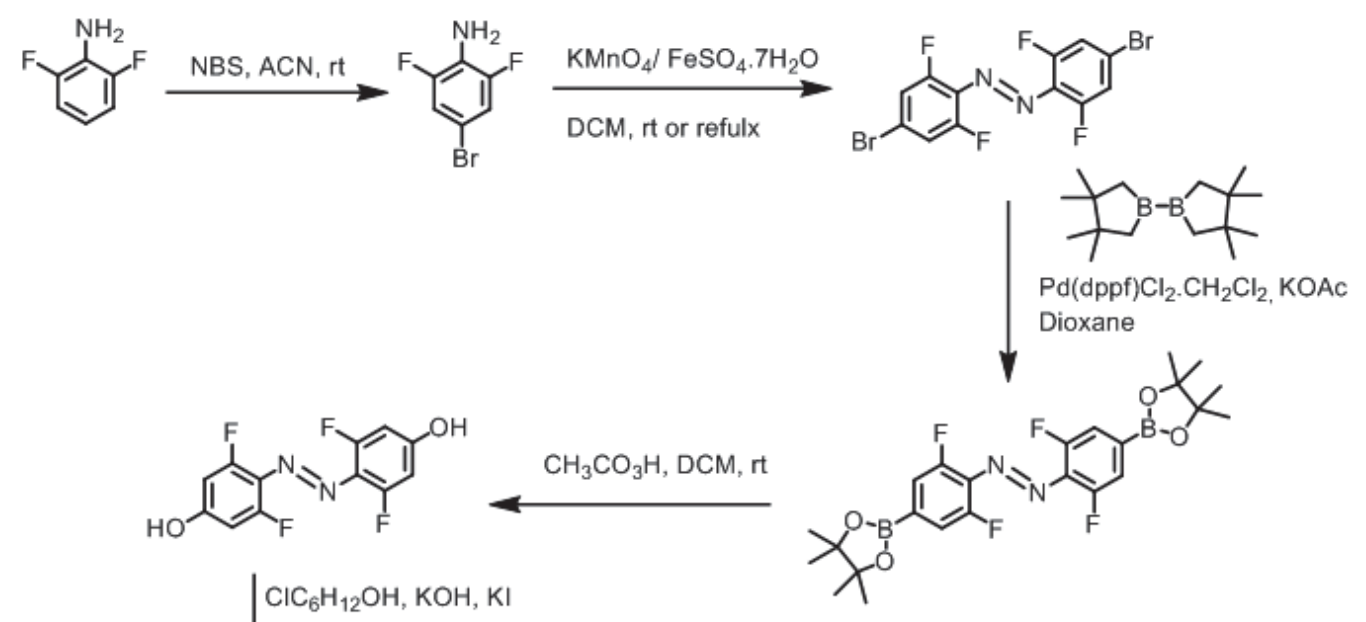

$\downarrow \mathrm{EtOH} / \mathrm{H}_{2} \mathrm{O}$, reflux

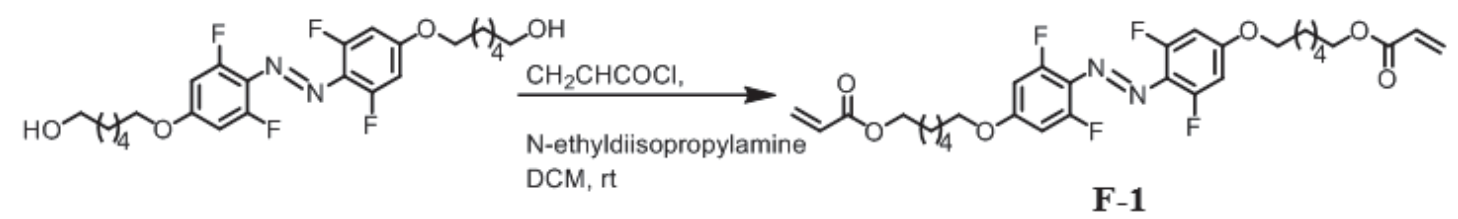

Scheme 4.1 Synthetic route of $o$-fluorobenzene monomer (F-1).

\subsubsection{Preparation of the ribbons}

The liquid crystal that was used contains $4.18 \mathrm{~mol} \%$ azo-switch monomer (either F-1 or H-1), $94.69 \mathrm{~mol} \%$ liquid crystal monomers (C6M:C6BP:C6BPN in molar ratios 1.17:2.96:1), $0.038 \mathrm{~mol} \%$ chiral dopant (S811) and $1.11 \mathrm{~mol} \%$ photo-initiator (Irgacure 819). The amount of chiral dopant $\mathbf{S 8 1 1}$ was adjusted to induce a cholesteric structure with $50 \mu \mathrm{m}$ quarter pitch. All components were mixed by dissolution in dichloromethane and further the solvent was evaporated on a hot plate at $60^{\circ} \mathrm{C}$ under a nitrogen stream. After removal of solvent, the resulting mixture was heated up to isotropic phase $\left(80^{\circ} \mathrm{C}\right)$ for 30 min gradually cooled down to the nematic phase $\left(48^{\circ} \mathrm{C}\right)$, and finally injected in a liquid crystal cell treated with alignment layers. The mixture was then left to organize in the cell at $48^{\circ} \mathrm{C}$ for $1 \mathrm{~h}$, before being photo-polymerized with visible light for $3 \mathrm{~h}$, using an Edmund MI-150 high-intensity illuminator equipped with a cutoff filter $(\lambda \geq 420 \mathrm{~nm})$. The polymerized film was retained in glass cell at $55^{\circ} \mathrm{C}$ overnight to ensure full polymerization. Such procedure was applied for film preparation in both twist- and planar-configuration cell with $50 \mu \mathrm{m}$ and $15 \mu \mathrm{m}$, respectively. The twist-cell was opened by using a scalpel. Once opened, the liquid crystal 
Preserving the delayed relaxation of $o$-fluorinated azobenzenes in liquid crystal polymer networks

polymer film remained attached on one of the glass plates. The twist film remaining on the glass plate was cut into ribbons at different cutting angles $\varphi$, and their dimensions were typically $\sim 1 \mathrm{x} \sim 6 \mathrm{x} 0.05 \mathrm{~mm}$.

\subsubsection{Supplementary information}

Analysis of ratio of cis-F-1 at photo-stationary state and upon relaxation in solution by ${ }^{1} \mathrm{H}-\mathrm{NMR}$ and relaxation of cis-H-1 by UV-vis measurement.

a

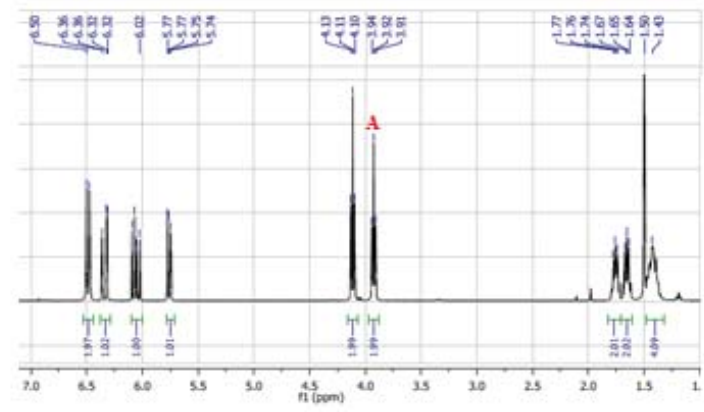

$\mathrm{b}$

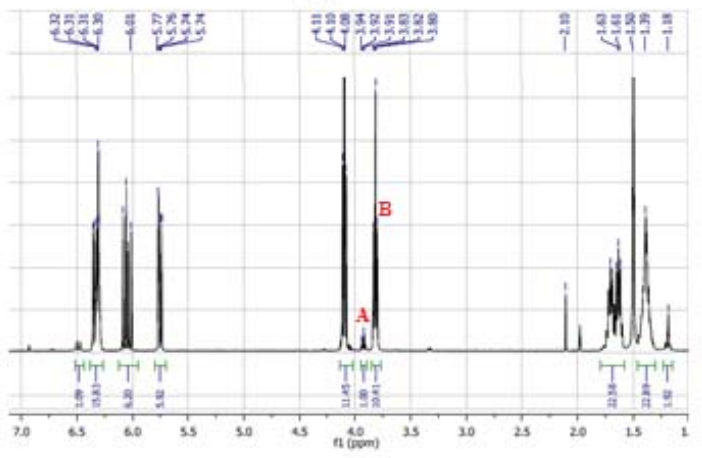

$\mathrm{c}$

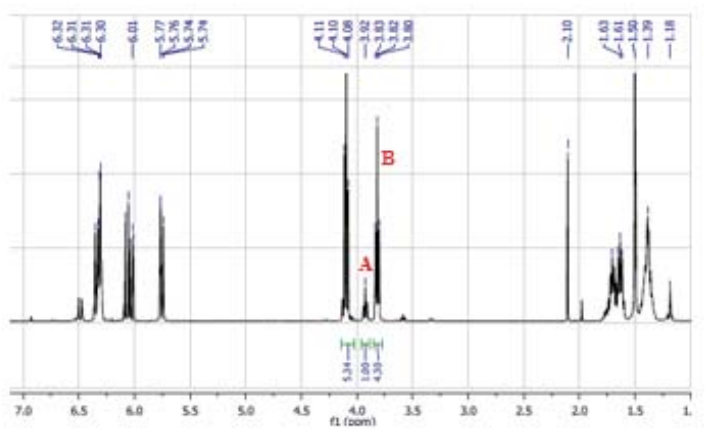

Figure 4.7 ${ }^{1} \mathrm{H}-\mathrm{NMR}$ spectra of $\mathbf{F}-\mathbf{1}$ in $\mathrm{CDCl}_{3}$ right after UV irradiation, and upon thermal relaxation in the dark. The ratio of cis-F-1 relative to trans-F-1 was determined by considering the integral of peak $\mathrm{B}$ ( $\delta 3.82)$ compared to $\mathrm{A}(\delta 3.92)$. a) before UV irradiation b) after $10 \mathrm{~min}$ irradiation with UV light $\left(77 \mathrm{~mW} / \mathrm{cm}^{2}\right)$ c) 5 days after $\mathrm{UV}$ irradiation was stopped. 


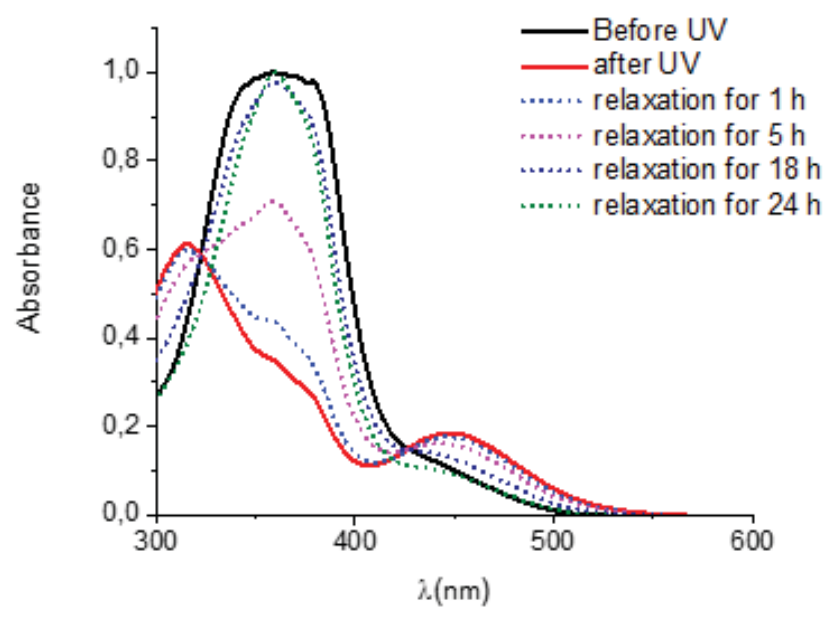

Figure 4.8 UV-vis spectra of $\mathbf{H}-1$ in $\mathrm{CH}_{2} \mathrm{Cl}_{2}\left(10^{-4} \mathrm{M}\right)$ before irradiation, after UV-irradiation (20 $\mathrm{min}, 2.21 \mathrm{~mW} / \mathrm{cm}^{2}$ ), and upon thermal relaxation in the dark.

\subsection{References}

$1 \quad$ Smith, K. K.; Kier, W. Am. Sc. 1989, 77, 28.

2 Gerbode, S. J.; Puzey, J. R.; McCormick, A. G.; Mahadevan, L. Science 2012, 337, 1087.

3 Qin, Z.; Buehler, M. J. Nat. Mater. 2013, 12, 185.

$4 \quad$ Vogel, V. Nat. Mater. 2012, 11, 841.

$5 \quad$ Rus, D.; Tolley, M. T. Nature 2015, 521, 467.

$6 \quad$ Kim, S.; Laschi, C.; Trimmer, B. Trends. Biotechnol. 2013, 31, 287.

7 Anderson, I. A.; Gisby, T. A.; McKay, T. G.; O’Brien, B. M.; Calius, E. P. J. Appl. Phys. 2012, 112, 041101.

8 Bauer, S.; Bauer-Gogonea, S.; Graz, I.; Kaltenbrunner, M.; Keplinger, C.; Schwödiauer, R. Adv. Mater. 2014, 26, 149.

9 Martinez, R. V.; Glavan, A. C.; Keplinger, C.; Oyetibo, A. I.; Whitesides, G. M. Adv. Funct. Mater. 2014, 24, 3003.

10 de Gennes, P. G.; Prost, J. The Physics of Liquid Crystals; Oxford University Press, Oxford, 1993.

11 Jiang, H.; Li, C.; Huang, X. Nanoscale 2013, 5, 5225.

12 Fleischmann, E.-K.; Zentel, R. Angew. Chem. Int. Ed. 2013, 52, 8810.

13 Xie, P.; Zhang, R. J. Mater. Chem. 2005, 15, 2529. 
Preserving the delayed relaxation of $o$-fluorinated azobenzenes in liquid crystal polymer networks

14 Warner, M.; Terentjev, E. M. Liquid Crystal Elastomers Oxford Univ. Press, 2003.

15 de Haan, L. T.; Schenning, A. P. H. J.; Broer, D. J. Polym. J. 2014, 55, 5885.

16 Urayama, K. React. Funct. Polym. 2013, 73, 885.

17 de Haan, L. T.; Verjans, J. M. N.; Broer, D. J.; Bastiaansen, C. W. M.; Schenning, A. P. H. J. J. Am. Chem. Soc. 2014, 136, 10585.

18 Ware, T. H.; McConney, M. E.; Wie, J. J.; Tondiglia, V. P.; White, T. J. Science 2015, 347, 982.

19 van Oosten, C. L.; Bastiaansen, C. W. M.; Broer, D. J. Nat. Mater. 2009, 8, 677.

20 Iqbal, D.; Samiullah, M. Materials 2013, 6, 116.

21 Ube, T.; Ikeda, T. Angew. Chem. Int. Ed. 2014, 53, 10290.

22 Ikeda, T.; Mamiya, J.-i.; Yu, Y. Angew. Chem. Int. Ed. 2007, 46, 506.

23 Iamsaard, S.; Aßhoff, S. J.; Matt, B.; Kudernac, T.; CornelissenJeroen, J. L. M.;

Fletcher, S. P.; Katsonis, N. Nat. Chem. 2014, 6, 229.

24 Eelkema, R.; Feringa, B. L. Org. Biomol. Chem. 2006, 4, 3729.

25 Yue Zhao, T. I. Smart Light-Responsive Materials: Azobenzene-Containing Polymers and Liquid Crystals; John Wiley \& Sons, Inc., Hoboken, New Jersey, 2009.

26 Harris, K. D.; Cuypers, R.; Scheibe, P.; van Oosten, C. L.; Bastiaansen, C. W. M.; Lub, J.; Broer, D. J. J. Mater. Chem. 2005, 15, 5043.

27 Liu, D.; Broer, D. J. Nat. Commun. 2015, 6.

28 Ilievski, F.; Mazzeo, A. D.; Shepherd, R. F.; Chen, X.; Whitesides, G. M. Angew. Chem. Int. Ed. 2011, 50, 1890.

29 Martinez, R. V.; Branch, J. L.; Fish, C. R.; Jin, L.; Shepherd, R. F.; Nunes, R. M. D.; Suo, Z.; Whitesides, G. M. Adv. Mater. 2013, 25, 205.

30 Vaia, R.; Baur, J. Science 2008, 319, 420.

31 Lendlein, A.; Langer, R. Science 2002, 296, 1673.

32 Corbett, D.; Warner, M. Liq. Cryst. 2009, 36, 1263.

33 Ionov, L. Langmuir 2015, 31, 5015.

34 Garcia-Amoros, J.; Finkelmann, H.; Velasco, D. J. Mater. Chem. 2011, 21, 1094.

35 Min Lee, K.; Lynch, B. M.; Luchette, P.; White, T. J. J. Polym. Sci. A Polym. 2014, $52,876$. 
36 Bobrovsky, A.; Shibaev, V.; Cigl, M.; Hamplova, V. r.; Hampl, F.; Elyashevitch, G. J. Mater. Chem. C 2014, 2, 4482.

37 Rullo, A.; Reiner, A.; Reiter, A.; Trauner, D.; Isacoff, E. Y.; Woolley, G. A. Chem. Commun. 2014, 50, 14613.

38 Bushuyev, O. S.; Tomberg, A.; Friščić, T.; Barrett, C. J. J. Am. Chem. Soc. 2013, $135,12556$.

39 Bléger, D.; Schwarz, J.; Brouwer, A. M.; Hecht, S. J. Am. Chem. Soc. 2012, 134, 20597.

40 Mossety-Leszczak, B.; Wlodarska, M.; Galina, H.; Bak, G. W. Mol. Cryst. Liq. Cryst. 2008, 490, 52.

41 Li, C.; Lo, C.-W.; Zhu, D.; Li, C.; Liu, Y.; Jiang, H. Macromol. Rapid Commun. 2009, 30, 1928. 
Preserving the delayed relaxation of $o$-fluorinated azobenzenes in liquid crystal polymer networks 


\section{Chapter 5}

\section{Twisting matter with light: engineering photo-switchable topological states}

Topology is not always intuitive and research efforts towards controlling and switching the topology of soft matter have appeared only recently. With the aim to achieve photo-control over the topological properties soft matter, here we demonstrate the writing and deleting of topological structures with light. Specifically, we report on the reversible topological structuring of chiral liquid crystals with light. By harnessing the action of chiroptical molecular switches that operate at the molecular scale, we show that a variety of twisted topological states can be generated locally, by using only subnanowatt optical power levels.

This chapter was published in: C. Loussert, S. Iamsaard, N. Katsonis and E. Brasselet, Adv. Mater. 2014, 26, 4242-4246. 
Twisting matter with light: engineering photo-switchable topological states

\subsection{Introduction}

Liquid crystals exhibit various kinds of topological defects that are associated to specific optical properties. These topological defects, smooth distortion of liquid crystal director over space, have been used in a number of applications, for example in integrated optical vortex generators. ${ }^{1-5}$ Topological defects also show potential towards information storage, because they cannot be transformed one to another by mere continuous changes of the material texture, which is why the writing and manipulation of topological defects in liquid crystals has been investigated recently. In particular, the pioneering work of Smalyukh et al. and the later work of Brasselet et al. on frustrated films of chiral liquid crystals have demonstrated that localized topological states can be written by either structured ${ }^{6}$ or unstructured ${ }^{7}$ light beams.

The use of light to induce localized topological states in chiral liquid crystals has paved the way towards their use for applications in terms of reconfiguration, ${ }^{6}$ large-scale integration, ${ }^{8}$ three dimensional photonic architectures, ${ }^{9}$ phase masks up to millimeter square area ${ }^{10}$ or arrays of microscopic optical vortex generators. ${ }^{11}$ However, in these early examples, the reversible generation of a localized defect structure by focused light beams typically requires a $10-100 \mathrm{~mW}$ power budget with typical intensities ranging from $1-10 \mathrm{~kW} \mathrm{~cm}^{-2}$ for low numerical aperture focusing elements up to $0.1-1 \mathrm{MW} \mathrm{cm}^{-2}$ for high numerical apertures. ${ }^{6,8}$ This implies a "point-by-point" laser writing strategy, and consequently implies long fabrication times for the realization of large and dense arrays. ${ }^{8,11}$ A few years ago, an approach relying on use of bulkyballs as dopants has been proposed to reduce the power budget by a few orders of magnitude, down to $10 \mu \mathrm{W} .{ }^{12}$ However, such an improvement came at the expense of reversibility, as intrinsically irreversible photo-chemical processes were involved. ${ }^{12}$ Combining optical sensitivity to reversibility would thus constitute a significant improvement for applications where addressability is a key issue.

Therefore, our molecular strategy relies on the use of chiroptical molecular motors. ${ }^{13,14}$ The use of light-responsive switches as molecular dopants for chiral liquid crystals has been reported before, and used successfully in controlling the pitch, handedness and orientation of the cholesteric helix, ${ }^{15}$ to convert light into macroscopic motion, ${ }^{16,17}$ or to develop photo- 
switchable surface topologies. ${ }^{18}$ However, to the best of our knowledge, they have not been used to control the structure of topological defects so far.

Here, we report on the use of chiral induction from light-driven molecular motors in order to achieve reversible topological structuring over films of frustrated chiral liquid crystals, by using subnanowatt light powers only. In so doing, we also demonstrate the formation of various kinds of chiral topological states at the micron-scale. Finally, our results unlock inherent limitations associated to the molecular photo-activation of textures using spatially extended illumination, see Ref. ${ }^{[19]}$ for thin films with perpendicular alignment and Ref. ${ }^{[20]}$ for parallel boundary conditions.

\subsection{Results and discussion}

Experiments are made in $10 \mu \mathrm{m}$ thin films of cholesteric liquid crystals. Specifically, we use so-called "frustrated" thin films. When the twisted elastic distortions associated with the cholesteric helix are incompatible with the orientation that is imposed at the interface, molecular chirality is not expressed and the director field is unwound. ${ }^{21}$ Such a "geometric frustration" occurs when the boundary conditions on both sides of the film promote a perpendicular (homeotropic) orientation of the director. More precisely, for a film of thickness $d$ with homeotropic alignment, the cholesteric helix is unwound when the pitch $p$ $>p_{\mathrm{c}}$ (Figure 5.1b), but winds when $p<p_{\mathrm{c}}$ (Figure 5.1c), which gives rise to fingerprint-like textures that are visible by polarized optical microscopy. ${ }^{22}$ The unwinding/winding transition occurs for the critical thickness-to-pitch ratio $d / p_{\mathrm{c}}=K_{3} /\left(2 K_{2}\right),{ }^{23}$ where $K_{2}$ and $K_{3}$ are elastic constants that involves structural characteristics of the liquid crystal. Based on these considerations, we anticipated that starting from a frustrated geometry $\left(p>p_{c}\right)$, a lighttriggered pitch decrease would allow remote-controlling a winding transition that would lead to the formation of a variety of well-defined topological structures. 
Twisting matter with light: engineering photo-switchable topological states

(a)

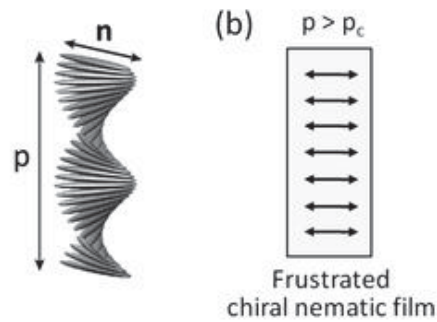

(c)

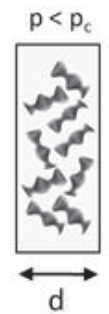

(d)

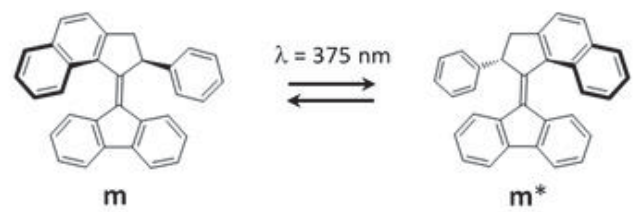

(e)

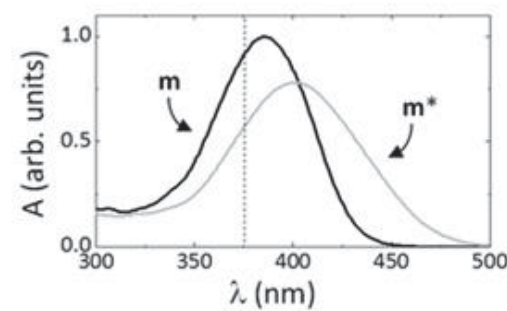

Figure 5.1 a) Representation of the helical ordering of a cholesteric liquid crystal, with $p$ the helical pitch and $n$ the director. b) Confinement of a cholesteric liquid crystal in a homeotropic cell of thickness $d$. The helical order is frustrated because $p>p_{c}$. In contrast, twisted defect textures appear when $p<p_{c}$ (c). Here the cholesteric pitch $p$ is optically adjusted by irradiation with light $(\lambda=375 \mathrm{~nm})$, that activates the molecular motor $\mathbf{m}$. The photo-chemical behavior of $\mathbf{m}$ is illustrated in panel $\mathbf{d}$ ) where $\mathbf{m}^{*}$ is the photo-activated isomer of $\mathbf{m}$. The photo-isomerization is associated with a change in helical twisting power of the motor e) UV-visible absorbance spectrum of the molecular switch $\left(10^{-4} \mathrm{M}\right.$ in hexane, room temperature). The black curve refers to the spectrum of $\mathbf{m}$ whereas the gray curve corresponds to the spectrum of $\mathbf{m}^{*}$.

We designed a cholesteric liquid crystal that winds under irradiation with light. Therefore we introduce a few percent of light-driven molecular motor $\mathbf{m}$ into an (achiral) nematic liquid crystal (the E7 mixture from Merck). Molecular motor $\mathbf{m}$ (Figure 5.1d) is known to undergo unidirectional rotation under irradiation with UV light $(\lambda=375 \mathrm{~nm}) .{ }^{24} \mathrm{In}$ liquid crystals, structural changes associated with the photoisomerization $\mathbf{m} \rightarrow \mathbf{m}^{*}$ translate into large cholesteric pitch variations ${ }^{25,26}$ (see absorption spectra of both forms in Figure 5.1e). In its stable form, $\mathbf{m}$ induces a right-handed cholesteric helix in E7 and, since we aim at optically reducing the pitch starting from an initial situation with $p>p_{c}$, a shape-persistent co-dopant inducing a left-handed twist is also added to the cholesteric mixture in order to overcompensate for the right-handed twist promoted by $\mathbf{m}$. We chose the shape-persistent chiral dopant (R)-2,2'-(1,3-propylenedioxy)-1,1'-binaphthalene. By using concentrations of 0.8 wt.\% and 1.0 wt.\% for the photo-responsive and shape-persistent chiral dopants respectively, our co-doping procedure yields a photo-responsive left-handed cholesteric liquid crystal that winds under irradiation with UV light, from the initial natural pitch $\mathrm{p}=18$ 
$\mu \mathrm{m}$ to $\mathrm{p}=1.7 \mu \mathrm{m}$ once photo-equilibrium is reached, which we evaluated from the GranjeanCano wedge method (Figure 5.2a; see also section 5.53). ${ }^{27}$ Since the elastic constants of E7 are $K_{2}=7.4 \mathrm{pN}$ and $K_{3}=16.5 \mathrm{pN}$ at $25^{\circ} \mathrm{C}$, we get $d / p_{c} \sim 1.1$ and we thus opt for a homeotropic cell of thickness $d=10 \mu \mathrm{m}$. As expected, the cholesteric helix is unwound at rest whereas frustration can be relieved under irradiation with UV light (Figure 5.2b).

a

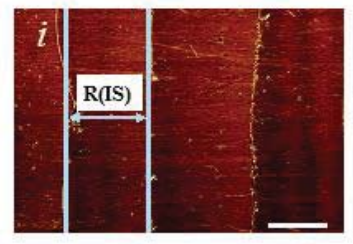

$\mathrm{b}$

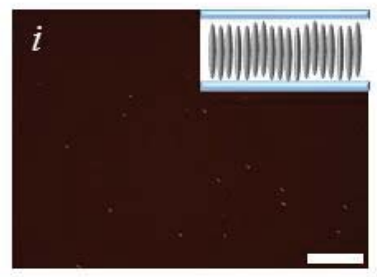

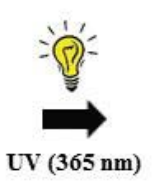
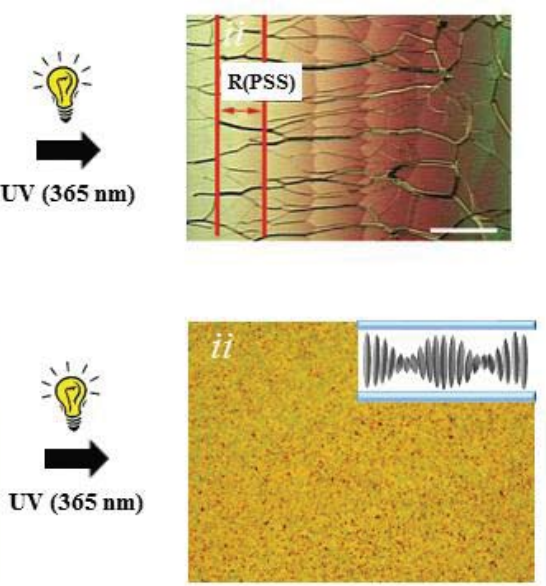

Figure 5.2 A light-responsive liquid crystal that undergoes winding (instead of unwinding) under irradiation with UV light. a) Reduction of distance between Cano's lines of the mixture in wedge cell under UV-illumination (30 $\mathrm{min}$ ). R(IS) and R(PSS) represent the distance between Cano's lines at initial and photo-stationary state, respectively. They implied pitch change of cholesteric structure, from $-18 \mu \mathrm{m}$ at initial state to $-1.7 \mu \mathrm{m}$ at photo-stationary state. b) texture modification of the mixture in homeotropic cell under UV-illumination (30 $\mathrm{min}$ ), from (i) nematic-like texture to (ii) fingerprinted texture; Inset of $b(i)$-(ii) depicting cholesteric structure at each state based on its texture under homeotropic confinement. Scale bar: $200 \mu \mathrm{m}$.

The setup developed by Dr. Brasselet (CNRS Bordeaux) allows using laser light $(\lambda=375$ $\mathrm{nm}$ ) by focusing the beam on the sample. Real-time monitoring of the light-induced topological structuring is performed with a CCD camera in front of which are placed a polarizer $\mathrm{P}$ and a filter $\mathrm{F}$ that blocks residual UV light. When the diameter of the beam $D$ reaching the sample is of the order of the thickness of the sample, distinct topological structures are formed $(D \sim d)$.

The photo-triggered transition to a wound state by using only subnanowatt power levels is ascertained experimentally by the appearance of a bright area against the black background (under crossed polarisers). Different kinds of photo-stationary states are found depending on the distance $\mathrm{z}$ between focal plane and sample as shown in Figures 5.3a-c that correspond to 
Twisting matter with light: engineering photo-switchable topological states

$\mathrm{D} \approx \mathrm{d}(\mathrm{z}=0), \mathrm{D} \approx 1.1 \mathrm{~d}(\mathrm{z}=100 \sim \mu \mathrm{m})$ and $\mathrm{D} \approx 2.6 \mathrm{~d}(\mathrm{z}=500 \mu \mathrm{m})$, respectively. Under visual inspection, these structures are reminiscent of topological objects called 'torons' and have been labeled as T3- $n(n=1,2,3)$ in Ref. ${ }^{[6]}$, where they were generated using high power light fields in passive cholesteric films. It should be noted that the structure shown Figure 5.3c might also be the manifestation of a Hopf fibration, ${ }^{28}$ which could be verified by a 3D reconstitution of the director field using fluorescence polarizing microscopy, but the experiment was not performed in the frame of this research effort.

The observation of distinct topological states calls for two comments. Indeed, one may rightly argue that: (i) in Ref. ${ }^{[6]}$, the laser-induced defect structures correspond to metastable distorted orientational states whereas our system, being fully reversible, eventually relaxes to the frustrated state after the writing light field is turned off and (ii) the microscopy images under crossed-polarizers shown in Figures 5.3a-c quantitatively differ from those shown in Ref. $^{[6]}$.

To address these two points, we compare our results to similar experiments performed in a $10 \mu \mathrm{m}$-thick frustrated film prepared by doping the nematic MLC-2048 with the chiral compound S811 (0.86 wt.\%) to provide a $10.4 \mu \mathrm{m}$-pitch passive cholesteric material. ${ }^{7}$ In that case, distinct metastable defect structures are observed and they do not disappear once the light beam is turned off (Fig. 5.3d-f). The topological structures that are observed unambiguously correspond to the same as created here in a photo-active cholesteric liquid crystal, which allows concluding that the twisted structures shown Figures 5.3a-c actually are topological microstructures. Still we note that the comparison of the crossed linear polarizers images of the structures we observe with the torons shown in Ref. ${ }^{[6]}$ reveals minor differences that are likely originating in different optical imaging conditions. 
Molecular optical activation in active chiral films
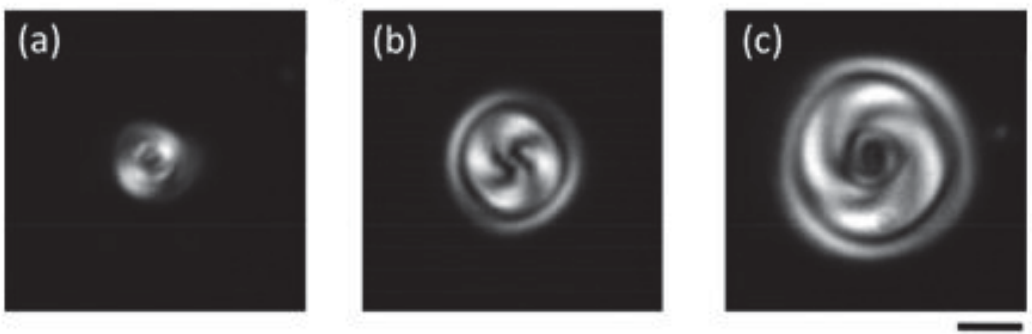

Supramolecular optical activation in passive chiral films
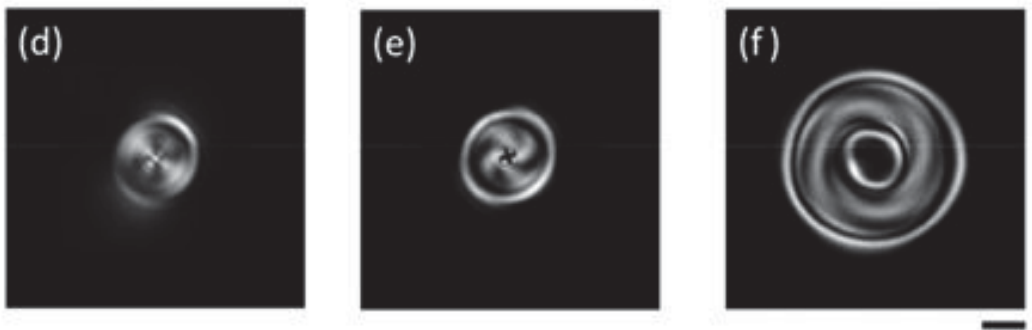

Supramolecular electrical activation in passive chiral films
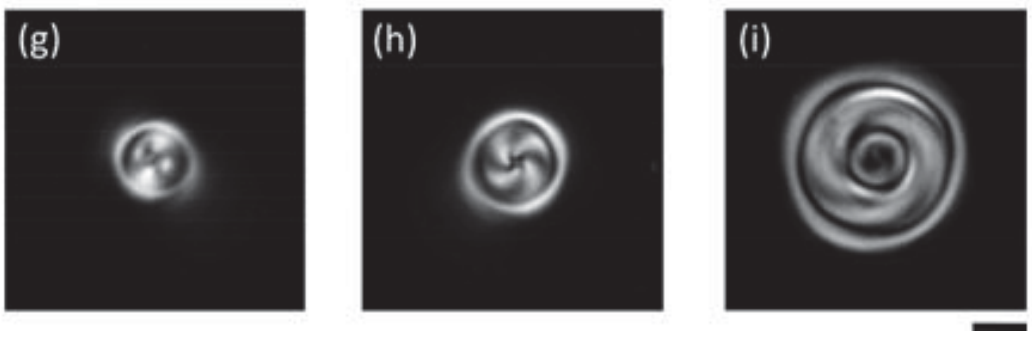

Figure 5.3 Topological structuring of a frustrated chiral nematic film. Upper row: local optomolecular triggering of defect structures in an active frustrated cholesteric film under UV illumination with a Gaussian beam. Experimental conditions: the beam waist diameter is $10 \mu \mathrm{m}$ and the sample is placed at a distance $\mathrm{z}=0,100$ and $500 \mu \mathrm{m}$ from the focal plane in panel (a), (b) and (c) that respectively correspond to the photo-stationary state under total incident power $0.53 \mathrm{nW}, 0.65 \mathrm{nW}$ and $1.04 \mathrm{nW}$. Middle row: local supramolecular optical activation of three different metastable structures in a passive frustrated cholesteric film under irradiation with a Gaussian beam of visible light, see also Ref [7] for details. Bottom row: spontaneous electrical generation of three different metastable structures in a passive frustrated cholesteric film. Scale bar: $10 \mu \mathrm{m}$. All images refer to crossed linear polarizers imaging.

As a comparison, we have also generated topological microstructures by using a uniform electric field as a trigger to achieve director reorientation. The $100 \mathrm{kHz}$ low-voltage electric field (a few Volts) is applied to indium tin oxide electrodes that are included in commercially available liquid crystal cells. In that case, the nematic liquid crystal has a negative dielectric anisotropy $\left(\epsilon_{a}=-3.4\right)^{29}$ that tends to reorient the director in the plane of the film. As when light is used as an external trigger, we observe the spontaneous electrical generation of a large 
Twisting matter with light: engineering photo-switchable topological states

number of randomly distributed distinct metastable defect structures across the sample. The structures shown in Figures 5.3g-i clearly match with the structures obtained using both lowpower UV writing in active films, and high-power visible writing in passive films.

Noticeably, the procedures we report on generating topological structures in frustrated films of liquid crystals support recent experiments ${ }^{7}$ demonstrating that helical beams do not constitute a pre-requisite to the formation of various kinds of chiral topological states, in contrast to state-of-the-art approach that relies on the use of high-order optical vortex beams in order to achieve topological diversity in passive media. ${ }^{6}$ Moreover, the fact that all structures have a typical size of the order of the pitch emphasizes that the observed topological diversity is inherent to the very nature of the chiral liquid crystal material and not of the used reorientation strategy, be it (i) local or spatially extended and (ii) acting at the molecular or supramolecular scale.

From a quantitative point of view, the opto-molecular approach developed here reduces both the required the intensity and power triggers by typically eight orders of magnitude compared to the case of passive materials. A physical insight into such an optical gain is inferred from comparing the optical cross-sections involved in the reorientation processes for either the passive or the photo-responsive films. For passive films, the typical length scale over which the optical stimulus (i.e., the torque exerted by light) is effective corresponds to the liquid crystal coherence length ${ }^{30}$ and is of the order of the film thickness $d$. In contrast, for active films, it is the light driven reversal of the molecular chirality that activates a chiral elastic torque at the molecular level, hence associated with a typical length scale $a \sim 1 \mathrm{~nm}$. Comparing the corresponding cross-sections areas yields a ratio $(d / a)^{2} \sim 10^{8}$, namely an improvement by eight orders of magnitude.

Finally, we have investigated the winding transitions that occur when the diameter of the writing beam is larger than the thickness of the photo-responsive film, i.e., $D>>$ d. Using spatially extended optical irradiation, the real-time monitoring of spatially controlled cholesteric textures, which are reminiscent of the well-known fingerprint textures, becomes possible. Arbitrary patterns with square centimeter area can be obtained under milliwatt power illumination since intensity level of the order of $1 \mathrm{~mW} \mathrm{~cm}^{-2}$ is sufficient. The demonstration of large area photo-patterning merely requires the use of a stencil used as a binary amplitude phase mask that is imaged on the sample. With the present material, photo- 
stationary patterns are typically obtained after $1 \mathrm{~min}$ irradiation, see Figure 5.4 where a checker-board pattern is shown. The obtained patterns emphasize the potential application in terms of image photo-inscription, although both the response time and image sharpness leave room for optimization. These results are consistent with previously reported observations, ${ }^{19}$ though the required intensity is here few tens times smaller.
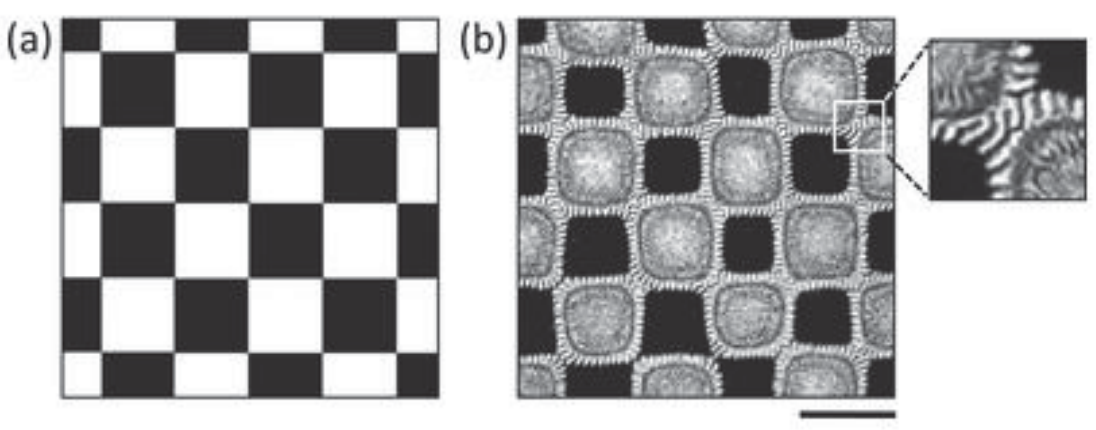

Figure 5.4 Steady-state checkerboard pattern image recorded under typical intensity of 1 $\mathrm{mW} \mathrm{cm} \mathrm{cm}^{-2}$. a) Original image of the amplitude mask. b) Imprinted pattern. The writing time of the order of $1 \mathrm{~min}$. Scale bar: $250 \mu \mathrm{m}$.

Importantly in terms of applicability, the patterning medium constituted by the photoresponsive material proved to be robust as it did not show significant sign of ageing after a few months, nor after many patterning/erasing cycles had been performed on the same sample. In addition, the all-optically driven processes are reversible and the kinetics of return to equilibrium are fully determined by the intrinsic properties of the photo-responsive chiral dopant. This means that the write-and-erase features of our opto-molecular addressing of liquid crystals can be tuned and optimized by appropriate engineering of the molecular switch. ${ }^{31}$ In particular, the use of photoactive chiral molecular switches with thermal stability is expected to overcome present limitations in terms of perennial recording of localized chiral topological states. ${ }^{32}$ 
Twisting matter with light: engineering photo-switchable topological states

\subsection{Conclusions}

We have demonstrated that topological states, either localized defects or textures, can be generated and modified with subnanowatt light power in chiral liquid crystals. Our strategy relies on photo-controlling chirality at the molecular scale. By achieving a drastic reduction of the required power budget with respect to state-of-the-art approaches, massively parallel optical writing of arbitrary localized microstructures can be envisioned using a light source with moderate power, for instance by using diffractive optical elements or lithographic exposure. Moreover, we showed that an optical winding transition can be triggered over large areas, which holds potential for scaling up. Our results also suggest that previous experiments involving the generation of topological structures and optical phase singularities based on chiral anisotropic fluids can be fruitfully revisited by using photo-responsive liquid crystals doped with molecular motors or switches.

\subsection{Acknowledgments}

We gratefully acknowledge Charles Loussert, our collaborator with the research team SINGULAR of Dr. Etienne Brasselet (University of Bordeaux, France) for performing the experiments on generating the topological defects and patterning our frustrated thin films of liquid crystals (Figure 5.3-5.4). We also acknowledge C. A. Hommersom for the synthesis of $(R)-2,2^{\prime}$-(1,3-propylenedioxy)-1,1'-binaphthalene.

\subsection{Materials and methods}

\subsubsection{General}

All chemical reagents used for the synthesis of the chiral dopants were purchased from Sigma-Aldrich. All liquid crystal cells were purchased from E.H.C. Co., Ltd, Japan. The nematic liquid crystal mixtures E7 and MLC-2048, and the chiral dopant S811 were supplied by Merck. UV-vis spectra were recorded on a Perkin Elmer Lambda 850 UV-vis spectrophotometer. ${ }^{1} \mathrm{H}$ NMR spectra of chiral dopants were recorded on a $400 \mathrm{MHz}$ spectrometer in $\mathrm{CDCl}_{3}$, and they were in accordance with literature data. 


\subsubsection{Synthesis and characterization of the chiral dopants}

Chiral dopant m, (R)-2,2'-(1,3-propylenedioxy)-1,1'-binaphthalene was synthesized according to a procedure adapted from Ref. ${ }^{[24]}$ and ${ }^{[33]}$ (Scheme 5.1). The two enantiomers of m were separated by chiral HPLC on a CHIRALPAK AD-H column using a methanol/ethanol (1:1) mobile phase, affording $>99 \%$ ee of enantiomer $\mathbf{m}$ with $(R, M)$ configuration and $>99 \%$ ee of enantiomer $\mathbf{m}$ with $(S, P)$-configuration, in a 1:1 ratio.

${ }^{1} \mathrm{H}-\mathrm{NMR}\left(400 \mathrm{MHz}, \mathrm{CDCl}_{3}\right): \delta 3.15\left(\mathrm{~d},{ }^{2} J_{\mathrm{HH}}=16.0 \mathrm{~Hz}, 1 \mathrm{H}, \mathrm{CH}_{2}\right), 3.91\left(\mathrm{dd},{ }^{2} J_{\mathrm{HH}}=16.0 \mathrm{~Hz}\right.$, $\left.{ }^{3} J_{\mathrm{HH}}=8 \mathrm{~Hz}, 1 \mathrm{H}, \mathrm{CH}_{2}\right), 5.30\left(\mathrm{~d},{ }^{3} J_{\mathrm{HH}}=8 \mathrm{~Hz}, 1 \mathrm{H}, \mathrm{CH}\right), 6.72-6.80(\mathrm{~m}, 2 \mathrm{H}), 7.02-7.20(\mathrm{~m}$, $7 \mathrm{H}), 7.24\left(\mathrm{t},{ }^{3} J_{H H}=7 \mathrm{~Hz}, 1 \mathrm{H}\right), 7.33-7.45(\mathrm{~m}, 4 \mathrm{H}), 7.69\left(\mathrm{~d},{ }^{3} J_{H H}=8 \mathrm{~Hz}, 1 \mathrm{H}\right), 7.73\left(\mathrm{~d},{ }^{3} J_{H H}=\right.$ $4 \mathrm{~Hz}, 1 \mathrm{H}), 7.80\left(\mathrm{~d},{ }^{3} J_{H H}=8 \mathrm{~Hz}, 1 \mathrm{H}\right), 7.87\left(\mathrm{~d},{ }^{3} J_{H H}=8 \mathrm{~Hz}, 1 \mathrm{H}\right), 7.93\left(\mathrm{~d},{ }^{3} J_{H H}=8 \mathrm{~Hz}, 1 \mathrm{H}\right)$. The helical twisting power (HTP) of $\mathbf{m}$ with ( $S, P$-configuration) was measured in E7 using a Grandjean-Cano wedge cell and provided the following values: $\operatorname{HTP}_{\mathrm{wt}}(\mathbf{m})=+66.04 \mu \mathrm{m}^{-1}$, $\operatorname{HTP}_{\mathrm{wt}}\left(\mathbf{m}^{*}\right)=-41.9 \mu \mathrm{m}^{-1}$.

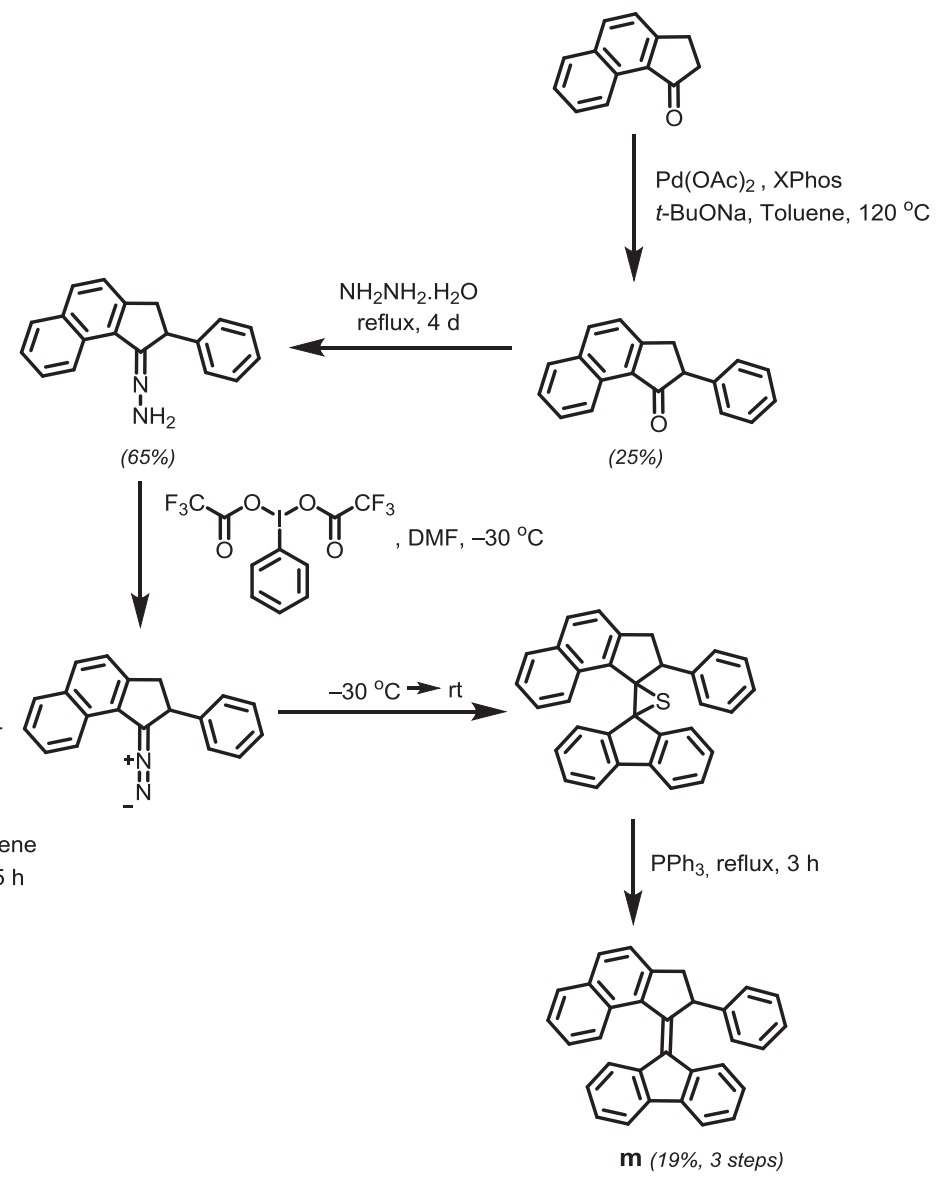

Scheme 5.1 Synthetic route of photo-active dopant $\mathbf{m} .{ }^{24,33}$ 
Twisting matter with light: engineering photo-switchable topological states

Shape-persistent chiral dopant $(R)-2,2$ '-(1,3-propylenedioxy)-1,1'-binaphthalene (Figure 5.5) was synthesized as described earlier in Ref. ${ }^{[34]} .{ }^{1} \mathrm{H}-\mathrm{NMR}(400 \mathrm{MHz}, \mathrm{CDCl} 3$ ): $\delta 7.96$ (d, 2H), $7.89(\mathrm{~d}, 2 \mathrm{H}), 7.47(\mathrm{~d}, 2 \mathrm{H}), 7.37$ (m, 2H), 7.22-7.28 (m, 4H), 4.31-4.44 (m, 4H), 1.94$1.99(\mathrm{~m}, 2 \mathrm{H})$. The HTP of $(R)-2,2$ '-(1,3-propylenedioxy)-1,1'-binaphthalene was measured in E7 using a Grandjean-Cano wedge cell and provided the value $\mathrm{HTP}_{\mathrm{wt}}=-65.5 \mu \mathrm{m}^{-1}$.

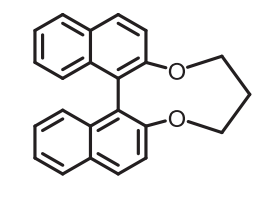

$\mathrm{BB} ;(R)$-configuration

Figure 5.5 Molecular structure of shape-persistent chiral dopant, $(R)-2,2^{\prime}-(1,3-$ propylenedioxy)-1,1'-binaphthalene.

\subsubsection{Preparation of the frustrated films of light-responsive liquid crystals}

Photo-inducing a decrease of pitch was a prerequisite to the approach we propose for generating twisted topological structures. However, currently, most light-responsive molecular motors and switches used as dopants induce a pitch increase of the cholesteric helix under irradiation with light. ${ }^{32,35-37} \mathrm{We}$ consequently designed a twisting cholesteric liquid crystal (Figure 5.6) by implementing a co-doping strategy that involves both a photoswitch to optically initiate structural modification and a chiral co-dopant that is shape persistent. Specifically, we used motor $\mathbf{m}(S, P)$ as the photo-active chiral dopant, that induces a right-handed helical twist in its stable state and a left-handed helical twist at photostationary state. The bridged binol BB, that promotes a left-handed helical twist in E7 was added. This co-dopant over-compensates the helical twisting power of $\mathbf{m}$ initially, but enhances the helical twisting power of $\mathbf{m}^{*}$ at photo-stationary state. After optimization of the doping ratios, this approach yielded a left-handed cholesteric liquid crystal that undergoes twisting, transitioning from a larger pitch to a smaller pitch under irradiation with light (Figure 5.6). 


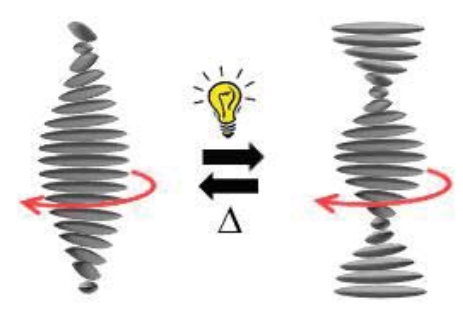

Figure 5.6 Schematic representation for the light-induced twisting of the cholesteric liquid crystal we have designed. The red arrow indicates the handedness.

The doping ratios for the two dopants was optimized by using equations 3-5 that allow pre-defining the values of the pitch at initial state and at photo-stationary state. The equations 3-5 were derived from common equations of induced cholesteric liquid crystal with multiple chiral dopants; $\mathbf{p}=\frac{1}{\text { C.HTP.ee }}$ (eq.1) and HTP $=\sum_{\mathbf{i}} \mathbf{X}_{\mathbf{i}} \mathbf{H T P}_{\mathbf{i}}$ (eq. 2), where $\mathbf{p}$ is cholesteric pitch, c is concentration of chiral dopants, HTP is overall helical twisting power of chiral dopants, $\boldsymbol{e} \boldsymbol{e}$ is enantiomeric excess, $\mathbf{H T P}_{\mathbf{i}}$ is helical twisting power of individual dopant $\mathrm{i}$, which is the efficiency of a dopant to twist a nematic liquid crystal and $\mathrm{X}_{\mathrm{i}}$ is weight fraction of individual dopant i relative to quantity of all chiral dopants used.

$$
\begin{aligned}
\mathbf{X}_{1} & =\frac{\mathrm{HTP}_{2} \cdot \frac{\mathbf{p}(\mathrm{PSS})}{\mathbf{p}(\mathrm{IS})}-\mathrm{HTP}_{2}}{\left[\mathrm{HTP}_{1}(\mathrm{IS})-\mathrm{HTP}_{2}\right]-\left[\mathrm{HTP}_{1}(\mathrm{PSS})-\mathrm{HTP}_{2}\right] \cdot \frac{\mathbf{p}(\mathrm{PSS})}{\mathbf{p}(\mathrm{IS})}} \\
\mathbf{C}_{\text {dopant }} & =\frac{1}{\mathbf{p}(\mathrm{IS}) \cdot\left[\mathrm{HTP}_{2}+\mathbf{X}_{1} \cdot\left[\mathrm{HTP}_{1}(\mathrm{IS})-\mathrm{HTP}_{2}\right]\right]} \\
\mathbf{C}_{1} & =\mathbf{X}_{1} \mathbf{C}_{\text {dopants }}
\end{aligned}
$$

Here, $\mathbf{X}_{\mathbf{1}}$ denotes the weight fraction of photoactive dopant $\mathbf{m}$ relative to quantity of both dopants, HTP1(IS) and HTP1(PSS) are helical twisting power of photoactive dopant at initial state $(\mathbf{m})$ and at photostationary state $\left(\mathbf{m}^{*}\right)$, respectively, $\mathbf{H T P} \mathbf{P}_{2}$ is helical twisting power of shape-persistent dopant $\mathbf{B B}, \mathbf{p}(\mathbf{I S})$ and $\mathbf{p ( P S S )}$ are pitch at initial- and photostationary state, respectively, $\mathrm{C}_{\mathrm{dopant}}$ is weight fraction of both dopants relative to quantity of all components, including chiral dopants and liquid crystal E7.

For the experiments described in this chapter we set the twisting range between $-10 \mu \mathrm{m}$ and $-1 \mu \mathrm{m}$, which involves doping ratios of $0.8 \% \mathrm{wt}$ of $\mathbf{m}$ and $1 \% \mathrm{wt}$ of $\mathbf{B}$ in E7. The liquid crystal mixture was prepared by dissolving both dopants into E7 with dichloromethane, and 
Twisting matter with light: engineering photo-switchable topological states

then evaporating the solvent at $60^{\circ} \mathrm{C}$ under a nitrogen stream. Finally the mixture was heated up to $80^{\circ} \mathrm{C}$ (over the isotropic temeprature) and gradually cooled down to ambient temperature. The resulting light-responsive cholesteric liquid crystal mixture was then introduced into liquid crystal cells. The photo-response of the liquid crystal was investigated by following the variation of the pitch as evidenced from a wedge cell (Figure 5.2a) and by monitoring texture transitions in a homeotropic cell under UV irradiation (Figure 5.2b) and thermal relaxation (Figure 5.6).
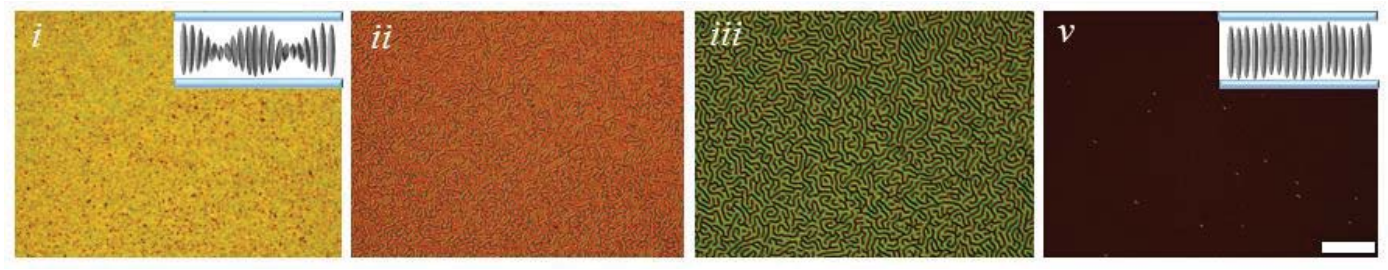

Figure 5.7 Texture modification during thermal relaxation of the light-responsive liquid crystal in a homeotropic cell. Scale bar: $200 \mu \mathrm{m}$.

\subsection{References}

1 Brasselet, E.; Murazawa, N.; Misawa, H.; Juodkazis, S. Phys. Rev. Lett. 2009, 103, 103903.

2 Brasselet, E.; Loussert, C. Opt. Lett. 2011, 36, 719.

3 Brasselet, E. Phys. Rev. Lett. 2012, 108, 087801.

4 Barboza, R.; Bortolozzo, U.; Assanto, G.; Vidal-Henriquez, E.; Clerc, M. G.; Residori, S. Phys. Rev. Lett. 2012, 109, 143901.

$5 \quad$ Loussert, C.; Delabre, U.; Brasselet, E. Phys. Rev. Lett. 2013, 111, 037802.

6 Smalyukh, I. I.; Lansac, Y.; Clark, N. A.; Trivedi, R. P. Nat. Mater. 2010, 9, 139.

$7 \quad$ Loussert, C.; Brasselet, E. Appl. Phys. Lett. 2014, 104, 051911.

8 Smalyukh, I. I.; Kaputa, D.; Kachynski, A. V.; Kuzmin, A. N.; Ackerman, P. J.; Twombly, C. W.; Lee, T.; Trivedi, R. P.; Prasad, P. N. Opt. Express 2012, 20, 6870.

9 Evans, J. S.; Ackerman, P. J.; Broer, D. J.; van de Lagemaat, J.; Smalyukh, I. I. Phys. Rev. E 2013, 87, 032503.

10 Ackerman, P. J.; Qi, Z.; Lin, Y.; Twombly, C. W.; Laviada, M. J.; Lansac, Y.; Smalyukh, I. I. Sci. Rep. 2012, 2, 414.

11 Bin, Y.; Etienne, B. J. Opt. 2013, 15, 044021. 
12 Trushkevych, O.; Ackerman, P.; Crossland, W. A.; Smalyukh, I. I. Appl. Phys. Lett. 2010, 97, 201906.

13 Feringa, B. L.; Huck, N. P. M.; Schoevaars, A. M. Adv. Mater. 1996, 8, 681.

14 B. L. Feringa, W. R. B. Molecular Switches; Wiley-VCH, Weinheim, Germany, 2011.

15 White, T. J.; Cazzell, S. A.; Freer, A. S.; Yang, D.-K.; Sukhomlinova, L.; Su, L.; Kosa, T.; Taheri, B.; Bunning, T. J. Adv. Mater. 2011, 23, 1389.

16 van Oosten, C. L.; Bastiaansen, C. W. M.; Broer, D. J. Nat. Mater. 2009, 8, 677.

17 Iamsaard, S.; Aßhoff, S. J.; Matt, B.; Kudernac, T.; Cornelissen, J. J. L. M.; Fletcher, S. P.; Katsonis, N. Nat. Chem. 2014, 6, 229.

18 Broer, D. J.; Bastiaansen, C. M. W.; Debije, M. G.; Schenning, A. P. H. J. Angew. Chem. Int. Ed. 2012, 51, 7102.

19 Gvozdovskyy, I.; Yaroshchuk, O.; Serbina, M.; Yamaguchi, R. Opt. Express 2012, 20,3499 .

20 Li, Q.; Li, Y.; Ma, J.; Yang, D.-K.; White, T. J.; Bunning, T. J. Adv. Mater. 2011, 23, 5069.

21 Randall, D. K.; Jonathan, V. S. J. Phys. Condens. Matter 2001, 13, R1.

22 Oswald, P.; Baudry, J.; Pirkl, S. Phys. Rep. 2000, 337, 67.

23 B. Y. Zel'dovich, N. V. T. JETP Lett 1981, 34, 406.

24 Vicario, J.; Walko, M.; Meetsma, A.; Feringa, B. L. J. Am. Chem. Soc. 2006, 128, 5127.

25 Eelkema, R.; Pollard, M. M.; Vicario, J.; Katsonis, N.; Ramon, B. S.; Bastiaansen, C. W. M.; Broer, D. J.; Feringa, B. L. Nature 2006, 440, 163.

26 Wang, Y.; Li, Q. Adv. Mater. 2012, 24, 1926.

27 P. Oswald, P. P. Nematic and Cholesteric Liquid Crystals: Concepts and Physical Properties Illustrated by Experiments; Taylor \& Francis, Boca Raton, FL, 2005.

28 Chen, B. G.-g.; Ackerman, P. J.; Alexander, G. P.; Kamien, R. D.; Smalyukh, I. I. Phys. Rev. Lett. 2013, 110, 237801.

29 Hsu, J.-S.; Liang, B.-J.; Chen, S.-H. Appl. Phys. Lett. 2006, 89, 051920.

30 Gennes, P. G. d. The Physics of Liquid Crystals; Clarendon Press, 1995, 2nd ed. 
Twisting matter with light: engineering photo-switchable topological states

31 Aßhoff, S. J.; Iamsaard, S.; Bosco, A.; Cornelissen, J. J. L. M.; Feringa, B. L.; Katsonis, N. Chem. Commun. 2013, 49, 4256.

32 Li, Y.; Xue, C.; Wang, M.; Urbas, A.; Li, Q. Angew. Chem. Int. Ed. 2013, 52, 13703.

33 Lubbe, A. S.; Ruangsupapichat, N.; Caroli, G.; Feringa, B. L. J. Org. Chem. 2011, 76, 8599.

34 van Es, J. J. G. S.; Biemans, H. A. M.; Meijer, E. W. Tetrahedron: Asymmetry 1997, 8,1825 .

35 Lub, J.; Nijssen, W. P. M.; Wegh, R. T.; De Francisco, I.; Ezquerro, M. P.; Malo, B. Liq. Cryst. 2005, 32, 1031.

36 Green, L.; Li, Y.; White, T.; Urbas, A.; Bunning, T.; Li, Q. Org. Biomol. Chem. 2009, 7, 3930 .

37 Li, Y.; Urbas, A.; Li, Q. J. Org. Chem. 2011, 76, 7148. 


\section{Chapter 6}

\section{Towards dual responsive liquid crystal films}

In Chapter 5, we have described how topological defects can be generated in liquid crystals, reversibly. While the generation of torons is now relatively well controlled, manipulating these defects remains a challenge, and would open perspectives towards precise, dynamic and continuous information units for optical function. One strategy to achieve this manipulation consists in creating the topological defects by using an electric field, and further use irradiation with light in order to induce rotation and/or translation of these defects. In this chapter, we design dual-responsive cholesteric liquid crystals that respond both to irradiation with light by a twist of the cholesteric pitch via transduction of molecular motion, and to an electric field, by a twist of the director that occurs by molecular reorientation. The design involves the use of photo-chemical switches, a co-dopant, and a liquid crystal matrix with relevant dielectric properties that accommodate the electric-field induced reorientation in the desired direction, under specific boundary conditions. 
Towards dual responsive liquid crystal films

\subsection{Introduction}

Defects are not always associated with negative phenomena. Various types of defects were observed in solid crystals ${ }^{1,2}$ and liquid crystals ${ }^{3-5}$ where they display fascinating physical properties.

In chiral nematic liquid in Chapter 5, I have discussed how in light-responsive liquid crystals confined in perpendicular boundary conditions, where the cholesteric helix is unwound and aligned perpendicularly to the substrate, ${ }^{6,7}$ a class of localized microstructures with twisted configuration called torons ${ }^{5}$ can be created by using an external trigger such as light or an electric field, by using either a supramolecular ${ }^{5,8}$ or a molecular-strategy (see also Figure 5.2). ${ }^{9,10}$ The former strategy by optically creating in passive film of untwisted cholesteric liquid crystal, called for high power of external field (in level of hundreds of $\mathrm{mW}){ }^{5,8}$ The strategy reported in Chapter 5 that is based on molecular activation, addresses this limitation by decreasing the energy required down to level of the nanowatt, based on the transduction of molecular movement into a twist of the director in the film. ${ }^{10}$

However, most of the work reported on torons so far described static torons. ${ }^{5,8,9}$ Mobile torons were mentioned briefly, ${ }^{11}$ but complex photo-control over the translation and rotation of torons remains to be demonstrated. Surprisingly, with a composition of photo-responsive liquid crystal similar as reported in Chapter 5, we have observed the continuous and regular rotation of torons upon increasing the power of light $(1.38 \mathrm{nW}) .{ }^{12}$ This preliminary observation inspired us to design novel dynamic features for localized structures. Ideally, these dynamic features can be implemented to provide information devices with new functions, in particular, in order to direct the transformation of optical information.

The engineering strategy is based on using one external stimulus (either e-field or light) to initially create the torons and the second stimulus to initiate and sustain their translation and rotation. Consequently, this strategy calls for the optimization of dual-responsive liquid crystals that respond both to irradiation with light and to an electric field, with twisting of the molecular director. Introducing such dual-responsiveness in confined untwisted cholesteric film was achieved by using liquid crystal containing a photo-active dopant to obtain lightresponsive liquid crystals. Meanwhile, for gaining additional access to an electrical response, the nematic liquid crystal used as a matrix for the system was a liquid crystal with negative 
dielectric anisotropy. The dipole moment of these liquid crystals is perpendicular to their long axis, which is why they tend to re-oriented perpendicularly to direction of the applied electric field (Figure 6.1a(ii)). This is a suitable behavior for the frustrated system we are considering, in which the liquid crystal is confined in homeotropic boundary condition initially (Figure 6.2). Specifically, we used a dual-frequency liquid crystal (DFNLC), ${ }^{13}$ constituting both positive and the required negative dielectric anisotropy at different frequencies that is characterized by a negative dielectric anisotropy above a cross-over frequency. Co-chiral dopants, one of which acts as a photo-active unit to twist the helix, and the other one being passive, were used to promote light induced twisting. As a preliminary investigation towards manipulating the torons, the formation of torons in such a liquid crystal is described hereafter.

\subsection{Results and discussion}

\subsubsection{A dual-responsive cholesteric liquid crystal and its photo- response under confinement}

As described earlier in Chapter 5, doping a liquid crystal with an efficient, chiral and photo-responsive dopant usually provides a cholesteric liquid crystal that unwinds under irradiation with light since many chiral photo-responsive dopants, designed to date, decrease order of liquid crystals upon their photo-isomerization that are accompanied by the reduction of helicity in liquid crystals. ${ }^{14}$ In order to design cholesteric liquid crystals that wind under irradiation with UV light, we consequently follow a co-doping strategy, where a lightresponsive dopant and a shape-persistent dopant are used in combination (see also Chapter 5; section 5.5.3). Here we use an overcrowded alkene as the photo-responsive dopant $\mathbf{m}$ (Fig. 6.1b) and a bridged binol BB as a shape-persistent dopant (Fig. 6.1c). These components are mixed in adequate proportions with a nematic liquid crystal matrix, to yield a photo-twisting cholesteric liquid crystal (Figure 6.1a). 
Towards dual responsive liquid crystal films

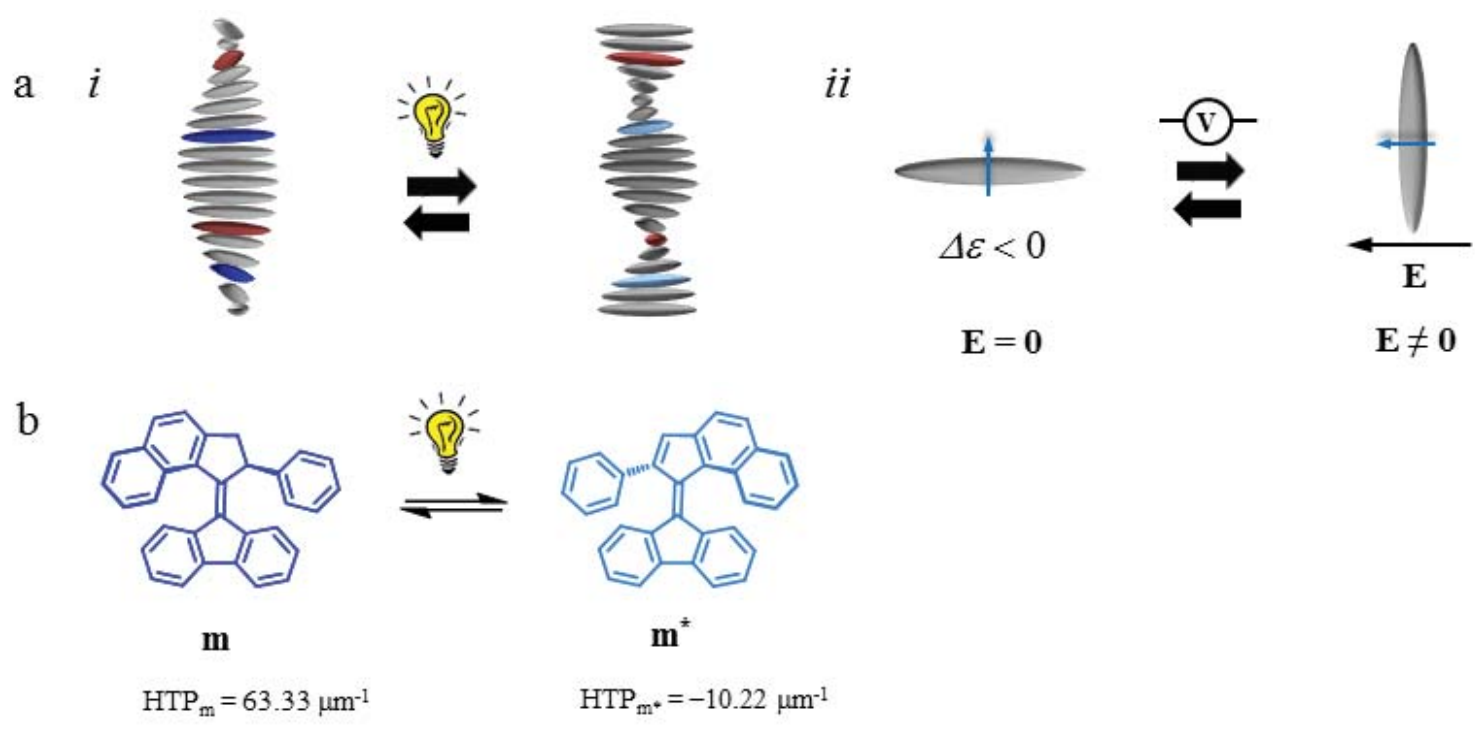

$\mathrm{c}$

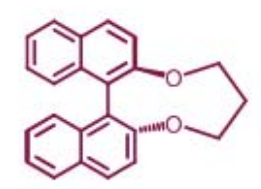

BB

$\mathrm{HTP}_{\mathrm{BB}}=-38.95 \mu \mathrm{m}^{-1}$

Figure 6.1 a) Responsiveness of dual-responsive liquid crystals by trigger of (i) light via photo-isomerization of chiral switch molecule or (ii) electric-field via interaction between liquid crystal medium and the applied electric field that is dictated by directions between transverse dipole moment of the liquid crystal with negative dielectric anisotropy (blue arrow) and applied electric field (black arrow).b) Molecular structure and photoisomerization of the photo-active dopant $\mathbf{m}$ c) Molecular structure of the shape persistent dopant $\mathbf{B B}$. ; distinct colors of dopants structures in $\mathrm{b}$ and $\mathrm{c}$ are relevant and used to recognize these dopants in a).

In order to design a dual-responsive liquid crystal that responds both to light and to an electric-field in particular confinement, the co-doping strategy described above is complemented by an appropriate choice of the nematic liquid crystal matrix, specifically, by choosing a nematic liquid crystals with relevant dielectric properties. In our study that involves frustrated cholesteric liquid crystals entrapped under homeotropic confinement (Figure 6.2a), the electric field should promote alignment parallel to the substrate, i.e. perpendicularly to the direction of the electric field, in order for a twisted structure to be formed (Figure 6.2). This condition imposes that the nematic liquid crystal should have a negative dielectric anisotropy where the required re-orientation of the director is induced by 
interaction between the transverse dipole moment of the liquid crystals on one hand and the direction of the applied field on the other hand (Figure 6.2b).

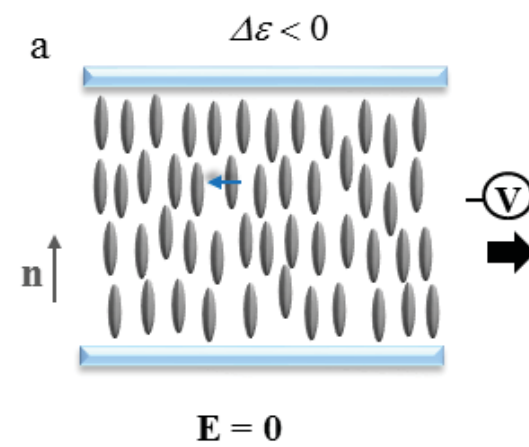

$\mathrm{b}$

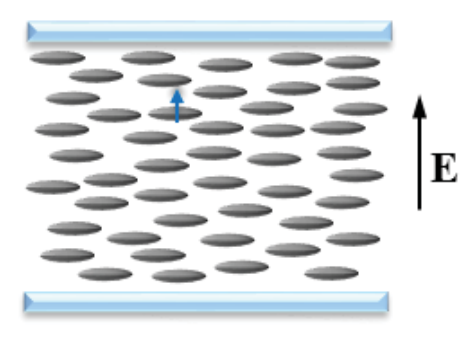

$\mathbf{E} \neq \mathbf{0}$

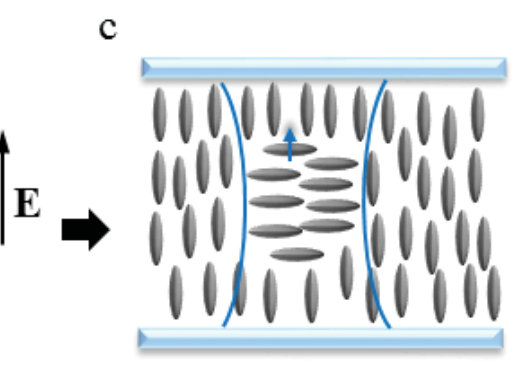

$\mathbf{E}=\mathbf{0}$

Figure 6.2 Re-orientation of liquid crystals with negative dielectric anisotropy $(\Delta \varepsilon<0)$ in homeotropic boundary condition by electric field a) initial untwisted state b) re-oriented state under the electric-field and c) residual state with the electrical-induced torons after the electric field is turned off. ; gray, blue and black arrows indicate the liquid crystal director (n), the transverse dipole moment and the direction of electric-field (E), respectively.

As liquid crystal host, we selected a commercially available liquid crystal that is nematic at room temperature (DFNLC), and whose untwisted cholesteric structure, formed after blending with chiral dopants and suppressing in homeotropic boundary condition, can be electrically tuned in two ways, i.e. both twisting and untwisting can be achieved. In the other words, the director of the initial untwisted cholesteric film in homeotropic boundary conditions can be re-oriented to twist and to reversibly untwist by choosing different frequencies of the electric field. The possibility of a two-ways switching is due to the special dielectric anisotropy of DFNLC matrix ${ }^{13}$ that is positive at low frequencies of the applied electric field, and becomes negative above a given cross over-frequency $\left(20 \mathrm{kHz}\right.$ at $\left.25^{\circ} \mathrm{C}\right)$. This two-way switching allowed to locally generate defect in confined untwisted cholesteric film (via twisting) as well as abruptly erase it (via untwisting) by adjusting the frequency of the electric field.

The proportions of chiral dopants were adjusted by using the equations that were described in Chapter 5 (section 5.5.3, eq 3-5). Using these equations required to predetermine the helical twisting powers (HTP) of the dopants in DFNLC, since HTP values, that characterize the capacity of chiral dopant to induce helical organization in liquid crystals (see also Chapter 5; section 5.5.3), depend both on the dopant and on the host matrix that are used. 
Towards dual responsive liquid crystal films

To this purpose, cholesteric liquid crystals were prepared by doping DFNLC with a chiral dopant, at different concentrations. The pitch of the resulting cholesteric liquid crystals was measured by using the Grandjean Cano method (using a wedge cell, see section 6.5.3). Knowing the concentration of the dopant (c) and the measured pitch (p), the HTPs could be measured by using a formula that is valid for low concentrations of dopants (HTP =1/c.p.ee). The HTP of photo-active dopant $(\mathbf{m})$ is $\operatorname{HTP}(\mathbf{m})=63.33 \mu \mathrm{m}^{-1}$ and $\operatorname{HTP}\left(\mathbf{m}^{*}\right)=-10,22 \mu \mathrm{m}^{-1}$ for the initial and the photo-stationary states, respectively (6.5.3, Table 6.1). The HTP of the shape persistent dopant $(\mathbf{B B})$ is $\operatorname{HTP}(\mathbf{B B})=-38.95 \mu \mathrm{m}^{-1}$ (6.5.3, Table 6.2). Additionally, the fact that these dopants promote an opposite handedness initially but the same handedness at photo-stationary state was confirmed by examining the direction shift of interference color of cholesteric films in wedge cell when turning analyzer from initial crossed position.

With these values of helical twisting powers in hand, the quantity of dopants ( $\mathbf{m}$ and $\mathbf{B B}$ ) needed for preparing dual-responsive cholesteric liquid crystals with different ranges of pitch change was derived from equations 3-5 shown in Chapter 5. In the following, we describe the behavior of two dual-responsive cholesteric liquid crystals that twist under irradiation with UV light, with a photo-induced pitch change that was pre-defined to range from -15.56 to $-5.50 \mu \mathrm{m}$ (liquid crystal $\mathbf{1}$ ) and from -18 to $-2 \mu \mathrm{m}$ (liquid crystal 2 ), respectively.

After introducing the cholesteric liquid crystals $\mathbf{1}$ and $\mathbf{2}$ into a cell with a $10 \mu \mathrm{m}$ gap and homeotropic anchoring conditions, both dual-responsive films displayed nematic-like textures under cross-polarizers. The absence of any fingerprint or finger texture confirmed that a helical twist was absent for both cholesteric liquid crystals in their ground state, being forced into an untwisted organization because of the anchoring conditions (Figure 6.3b(i) and $\mathrm{c}(i))$. Alternatively, under irradiation with light $(\lambda=365 \mathrm{~nm})$, the texture of both films was modified into a fingerprint, a pattern that is characteristic for twisted cholesteric structures (Figure 6.3b(ii) and c(ii)). This observation indicates that the photo-isomerization $\mathbf{m}$ into $\mathbf{m} *$ has modulated molecular interactions in the cholesteric system which eventually results in cholesteric restructuring. The characteristic textures of untwisted- and twisted cholesteric liquid crystals in homeotropic anchoring are well known to emerge from the competition between the chiral strength of the cholesteric liquid crystal and the anchoring strength of confinement, which can be engineered by adjusting the ratio between the cholesteric pitch $(p)$ and the thickness of cell $(d) ; d / p_{c}=K_{3} /\left(2 K_{2}\right)$ (see also Chapter 5). ${ }^{6,7}$ More simply, the 
smaller ratio of pitch relative to thickness, the more likely twisted structure that is recognized by fingerprint texture is developed. Consequently, the result of texture alteration in both liquid crystals (Figure 6.3b and c) reveals twisting of cholesteric structure from larger to shorter pitch by light activation (Figure 6.3a), which constitutes a pre-requisite to photogenerate defects.

a

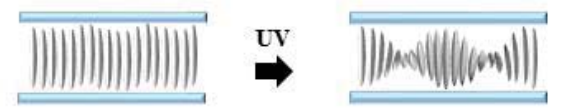

$\mathrm{b}$

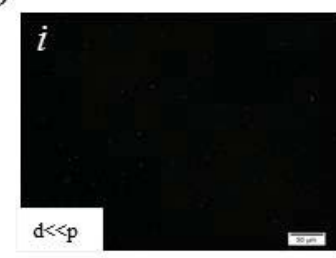

d

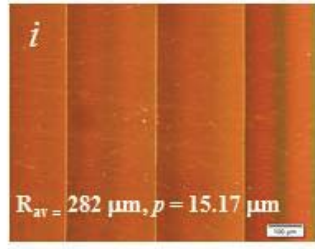

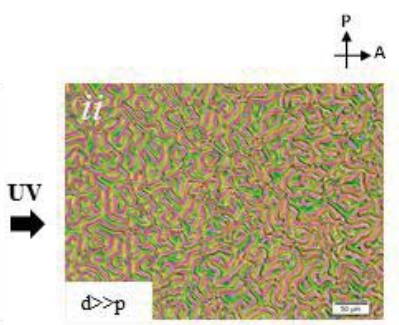

$\stackrel{p}{\uparrow} \mathrm{e}$

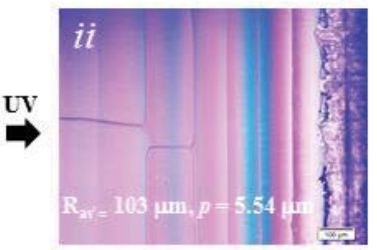

c
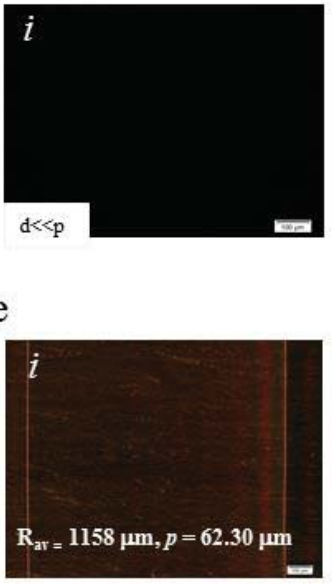

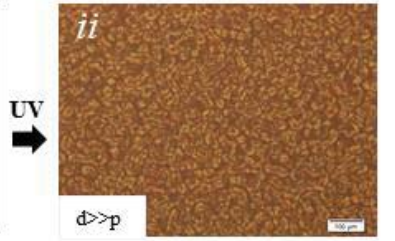

$\stackrel{P}{\rightarrow} A$
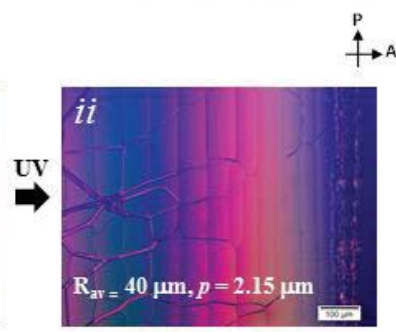

Figure 6.3 a) Illustration of structural modification of dual-responsive cholesteric liquid crystal in homeotropic confinement by light-induced twisting. Polarized optical micrographs of b) cholesteric liquid crystal $\mathbf{1}$ and c) cholesteric liquid crystal $\mathbf{2}$ in homeotropic confinement upon UV-exposure. Polarized optical micrographs of d) mixture 1 and e) mixture 2 in wedge cell $(\tan \theta=0.0269)$ upon UV-exposure. ; $i$ and ii indicating initial- and photo-stationary state (30 min UV-exposure), respectively. $R_{a v}$ standing for average distance between Cano lines that can be convert to pitch value $(p)$.

Using the Grandjean Cano method, it was possible to confirm that the cholesteric liquid crystal 1 exhibited pitch change from $-15.17 \mu \mathrm{m}$ (at initial state, IS) to $-5.54 \mu \mathrm{m}$ (at photostationary state, PSS) with high precision in respect to pre-defined values at both state, from $-15.56 \mu \mathrm{m}$ (IS) to $-5.50 \mu \mathrm{m}$ (PSS) (Figure 6.3d). The pitch of cholesteric liquid crystal 2 (Figure 6.3e) ranged from $-62.30 \mu \mathrm{m}$ (IS) to $-2.15 \mu \mathrm{m}$ (PSS) that showed large deviation relative to pre-defined value at the initial state (IS), $-18 \mu \mathrm{m}$, while closer to pre-defined value at the photo-stationary state (PSS), $-2 \mu \mathrm{m}$. The large deviation between the measured pitches of liquid crystal 2 compared to their pre-defined values is likely due to the fact that some 
Towards dual responsive liquid crystal films

twisting range fall where there is a non-linear relation between the doping concentration (c) and the inverse of the pitch $(1 / p)$, i.e. out of the expression $c=1 /(p . H T P . e e)$. In particular, the use of high concentrations of dopant which is naturally required for strong twists as for mixture 2, accounts for this non-linearity. ${ }^{15}$ As expected, twisted state (fingerprint texture) of cholesteric films under homeotropic anchoring condition could switch back to initial untwisted state (nematic-like texture) in the dark, returning to its initial state 20 minutes after irradiation was stopped (Figure 6.8), as directed by the back-isomerization of $\mathbf{m}^{*}$ to $\mathbf{m}$. The reversibility of the textures of the liquid crystal films demonstrated enabling ability for re-writing and self- or commanded erasing device.

\subsubsection{Creation of localized defects in thin films of dual responsive liquid crystals using light}

Cholesteric liquid crystal 2 was used to investigate the possibility to induce the rotation of optically-induced torons with light. In our early observations, the rotation of localized defects was observed upon increasing the power of light up to $1.38 \mathrm{nW}$ in a film that is identical to the light-responsive film reported in Chapter $5 .^{12} \mathrm{We}$ thus anticipated that it was also possible to induce the rotation of the torons in a dual- responsive cholesteric film. The rotation of topological defects likely originates from two factors; 1) a gradient of pitch between the defect and the surrounding bulk and 2) a continuous re-orientation of the director within the static defect induced by a gradual increase in the light beam intensity. The optical generation of the torons and other defects, and attempts to induce their rotation were performed by using a Gaussian light beam as in Chapter 5. Light was split into two paths (Figure 6.4a), including a wide diameter beam $\left(\mathrm{B}_{1}\right)$ that is used to control the background pitch, i.e. the natural pitch of the frustrated cholesteric liquid crystal, and a narrow beam $\left(\mathrm{B}_{2}\right)$ that is used to generate the defect locally and to induce its rotation as well. A range of illumination powers was used to determine the threshold over which the geometric frustration is relieved. At powers above $\sim 700 \mathrm{mkW}$, we observed that the frustrated cholesteric organization switched into a twisted state, as evidenced by a texture transition that can be followed by optical microscopy. In our attempts to induce the rotation of torons, we tried various choices between the natural pitch and the photo-generated pitch, by adjusting the power of the wide beam $\left(B_{1}\right)$ in a range below the unwinding threshold $(0-650 \mathrm{mkW})$ to 
control the pitch of the background. The pitch within the localized defect was expected to constantly reach minimum value (at $\sim 2 \mu \mathrm{m}$ (PSS) of liquid crystal 2) by using the narrow light beam $\left(\mathrm{B}_{2}\right)$. For each power of $\mathrm{B}_{1}$ (wide beam), localized defects were generated and transformed by gradually increasing the power of $\mathrm{B}_{2}$ (narrow beam) (Figure 6.4a).
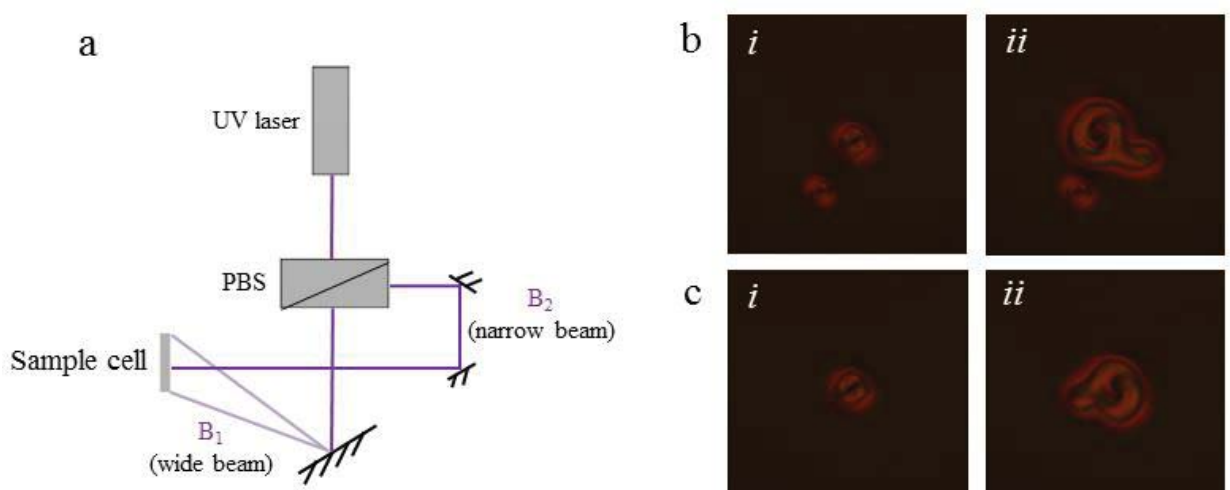

c
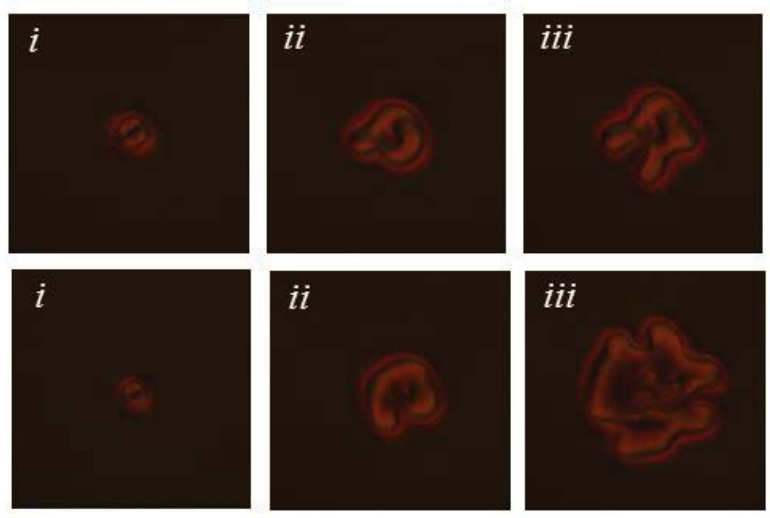

Figure 6.4 a) Experimental setup, demonstrating two splitting paths of light beam, $\mathrm{B}_{1}$ (background beam) and $\mathrm{B}_{2}$ (localized beam). b-d) Polarized optical micrographs of lightinduced defect creation and manipulation in the homeotropic film $2(\mathrm{~d}=10 \mu \mathrm{m})$ under pitch gradient condition by adjusting split beams b) $\mathrm{B}_{1}=650 \mathrm{mkW}$ and $\mathrm{B}_{2}=0 \rightarrow 1.59 \mathrm{nW}$ c) $\mathrm{B}_{1}$ $=450 \mathrm{mkW}$ and $\left.\mathrm{B}_{2}=0.9 \rightarrow 2.55 \mathrm{nW} \mathrm{d}\right) \mathrm{B}_{1}=0 \mathrm{mkW}$ and $\mathrm{B}_{2}=1.23 \rightarrow 5.43 \mathrm{nW}$.; $i$ at initial state, $i i$ at intermediate state and iii at final state upon increasing power of $\mathrm{B}_{2}$.

Based on relative pitches of these two regions, namely between background and the localized defect, generation and manipulation of defect with pitch gradient condition could be divided into three cases, according to set of background pitch, in which; 1) $\mathrm{p}_{\text {background }} \sim \mathrm{d}$, but $>$ p pthreshold $\left.\left.\left(B_{1}=650 \mathrm{mkW}\right) 2\right) p_{\text {background }}>>d\left(B_{1}=150-450 \mathrm{mkW}\right) 3\right) p_{\text {background }} \sim-62.3$ $\mu \mathrm{m}\left(\mathrm{B}_{1}=0 \mathrm{mkW}\right)$. The result of all cases are depicted in Figure 6.4b-d (and Figure 6.9, providing additional experiments of cases 2 ). In all cases, the torons were created statically in film at lower power of narrow beam $\left(\mathrm{B}_{2}\right)$ (initial applied power, Figure $6.4 \mathrm{~b}-\mathrm{d}(\mathrm{i})$ and 6.9(i)). However, their rotation was never detected, even when the power of the beam $\left(\mathrm{B}_{2}\right)$ was ranged up (Figure 6.4b-c(ii-iii) and 6.9(ii)). We anticipate that the absence of any rotation arises from the lack of control over the pitch, or was related to the physical properties of the 
dual-frequency nematic liquid crystal matrix. Instead, we observed transformation of localized microstructure from a well-defined toron into various cholesteric finger-like structures upon increasing the power of $\mathrm{B}_{2}$ gradually (Figure 6.4b-d). This shape evolution of localized microstructure based on the control of pitch gradient by light for all cases is shown Figure 6.5.
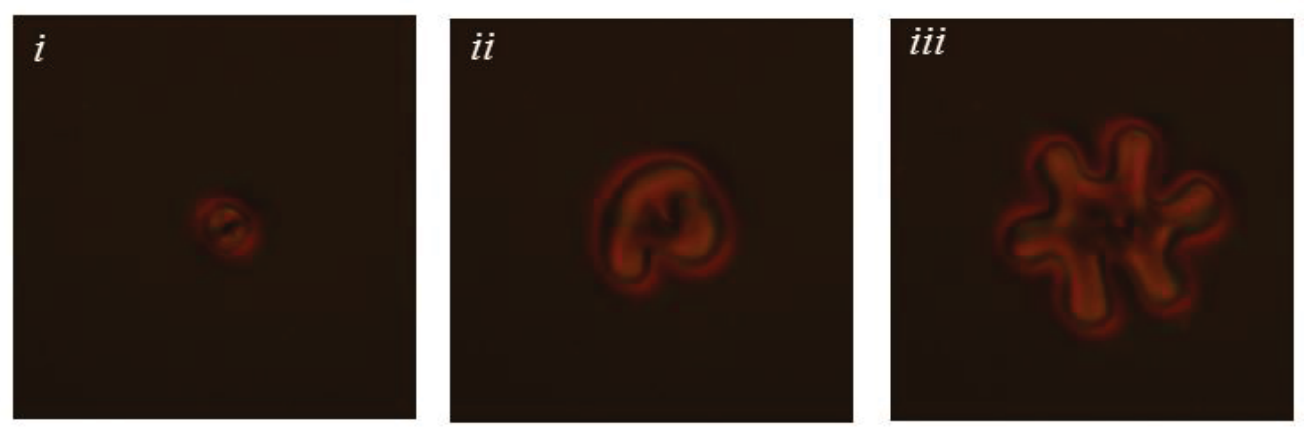

Figure 6.5 Shape evolution of a micro-twisted defect structure in 2 submitted to perpendicular boundary conditions. (case $2 ; \mathrm{B}_{1}=300 \mathrm{mkW}$ and $\mathrm{B}_{2}=0.9 \rightarrow 3.71 \mathrm{nW}$ )

\subsubsection{Creation of localized defects in thin films of dual responsive liquid crystals using electric field}

Future experiments will make use of the electrical-responsiveness of the dual frequency nematic matrix, by creating torons with an electric field (Figure 6.2) and either rotate them by irradiating with light locally, or translate them by inducing a gradient of pitch along the sample. Using electric-field is expected to stabilize the presence of the torons longer relative to creation by light ascribed to exclusion of thermal relaxation of $\mathbf{m}^{*}$ from the reversible process to untwisted state. The more stability of the electrical-induced torons may facilitate induction and manipulation of the defect rotation. 


\subsection{Conclusions and perspectives}

We report the design of a dual-responsive cholesteric liquid crystal that responds to both irradiation with light or to an electric-field, by twist of director. The design of this dualresponsive liquid crystal is based on the co-doping strategy that was already used successfully in Chapter 5 that involves using both light-responsive and shape-persistent dopants. The dual-twisting function of this cholesteric liquid crystal facilitates structure and texture modulation in homeotropic confinement, which revealed especially fruitful for designing adaptive boards to write twisted microstructures either optically or electrically. Also, the reversibility of twisting gave access to both erasing and re-writing functions. Our early attempts to create and optically manipulate topological defects, by exploiting a gradient of pitch between the localized defect and the surrounding bulk, however, only yielded a stepwise evolution from torons to cholesteric finger-like structures upon increasing the power of the localized light beam. Manipulating torons consequently remains an open challenge that we propose to address by either using different nematic liquid crystal hosts or by optimizing the range of the photo-induced pitch change.

\subsection{Acknowledgments}

We gratefully acknowledge Dr. Etienne Brasselet and his team "Singular optics and liquid crystals" (University of Bordeaux, France) and Dr. Tatiana Orlova for the experiments on topological defects (Figure 6.4, 6.5 and 6.9). C. A. Hommersom is acknowledged for the synthesis of (R)-2,2'-(1,3-propylenedioxy)-1,1'-binaphthalene.

\subsection{Materials and methods}

\subsubsection{General}

Both the wedge cells and the homeotropic cells were purchased from E.H.C. Co., Ltd, Japan. The nematic liquid crystal DFNLC was supplied by BEAM Engineering for Advanced Measurements Co. Dichloromethane with HPLC grade was purchased from VWR. Optical micrographs of texture and pitch characterization were taken with a polarized optical 
Towards dual responsive liquid crystal films

microscope Olympus (BX51). All illuminating experiments with wedge cell were carried out with a Spectroline $36-380 \mathrm{UV}$ lamp at $\lambda=365 \mathrm{~nm}\left(\approx 1.000 \mu \mathrm{W} / \mathrm{cm}^{2}\right)$. The laser illumination for defect generation was set at the University of Bordeaux, France.

\subsubsection{Preparation of the cholesteric liquid crystals}

The synthesis and characterization of chiral dopant $\mathbf{m}$ were previously described in chapter 5 (section 5.5.2, scheme 5.1). Likewise, all doped cholesteric liquid crystals were prepared by mixing each dopant with DFNLC in dichloromethane. The resulting solution was then evaporated at $60^{\circ} \mathrm{C}$ under a stream of nitrogen, providing a cholesteric liquid crystal. The liquid crystal was heated up to isotropic phase (transparent) at $\sim 110^{\circ} \mathrm{C}$ for $30-60 \mathrm{~min}$ to secure the homogeneity of the mixture, followed by gradually cooling down to ambient temperature. The cholesteric liquid crystal mixture was then ready to be injected into cell by capillary force. To determine HTP of dopants, cholesteric liquid crystals doped with single dopant either $\mathbf{m}$ or $\mathbf{B B}$ were prepared with different doping concentrations. Determining the HTP of the shape persistent dopant was performed by doping DFNLC with $0.5 \%$ and $0.25 \%$ of BB. Determining the HTP of the photo-active dopant was performed by doping DFNLC with $0.5 \%, 0.25 \%$ and $0.125 \%$ of $\mathbf{m}$. The dual-responsive liquid crystal 1 with a pitch that varies from $-15,56 \mu \mathrm{m}$ to $-5,50 \mu \mathrm{m}$ was prepared out of $0.16 \%$ wt of $\mathbf{m}$ and $0.42 \%$ wt of $\mathbf{B B}$ in DFNLC. The dual-responsive liquid crystal 2 with a pitch that varies from $-18 \mu \mathrm{m}$ to -2 $\mu \mathrm{m}$ was prepared out of $0.60 \%$ wt of $\mathbf{m}$ and $1.13 \%$ wt of BB in DFNLC. The values of pitch were measured by the Grandjean Cano method, using a wedge cell $(\mathrm{d}=0.7 \mathrm{~mm}, \tan \theta=$ 0.0269)). The values of pitch at photo-stationary state were also measured in a Grandjean Cano wedge cell, after 30 min illumination with UV light $(1.000 \mu \mathrm{W} / \mathrm{cm} 2)$. Cholesteric liquid crystals 1 and 2 were introduced in cells with homeotropic anchoring (with $10 \mu \mathrm{m}$ thickness) by capillarity. 


\subsubsection{Helical twisting power of dopants in a dual frequency nematic liquid crystal}

The helical twisting powers of the photo-active dopant $\mathbf{m}$ and the shape-persistent dopant BB were determined based on the following equation that is valid at low concentrations $(\mathrm{HTP}=1 /$ c.p.ee $)$.

a

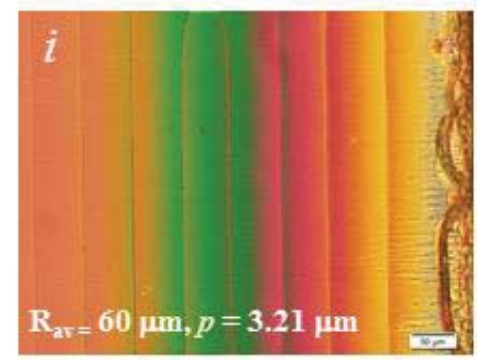

b

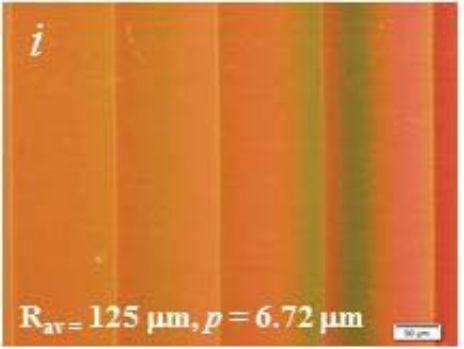

$\mathrm{c}$

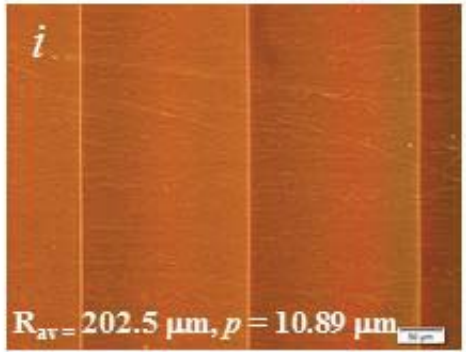

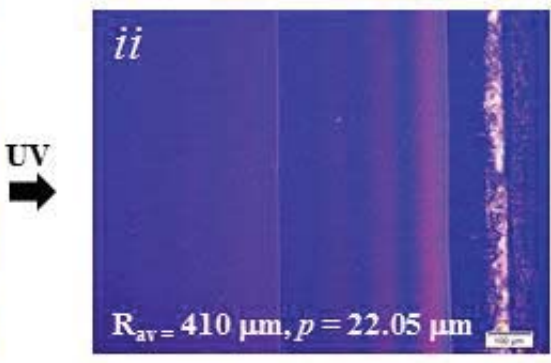
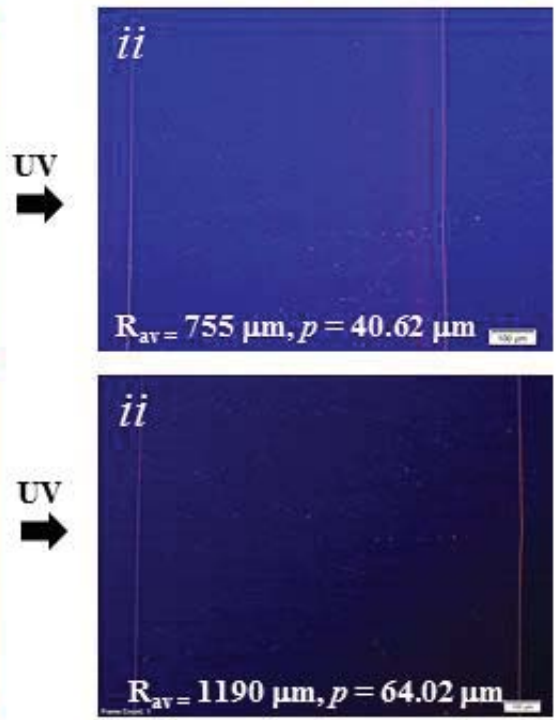

Figure 6.6 Polarized optical micrographs of Cano wedge cells of a) $0.5 \%$ wt, b) $0.25 w t \%$ and c) $0.125 \mathrm{wt} \%$ of chiral dopant $\mathbf{m}$ in DFNLC (i) at initial- and (ii) at photostationary state (after 30 min exposure to UV light).; $\mathrm{R}_{\mathrm{av}}$ standing for average distance between Cano lines that implied pitch $(p)$ of each mixture. 
Towards dual responsive liquid crystal films

Table 6.1 HTP calculated from different concentrations of $\mathbf{m}$ at initial- $\left(\mathrm{HTP}_{\mathrm{m}}\right)$ and photo stationary state $\left(\mathrm{HTP}_{\mathrm{m}}\right)$.

\begin{tabular}{|c|c|c|c|c|}
\hline Concentration of $\mathbf{m}$ in DFNLC & $p_{i}(\mu \mathrm{m})$ & $p_{i i}(\mu \mathrm{m})$ & $\mathrm{HTP}_{\mathrm{m}}(\mathrm{wt})$ & $\mathrm{HTP}_{\mathrm{m}}(\mathrm{wt})$ \\
\hline $0.5 \% \mathrm{wt}$ & 3.21 & 22.05 & 57,65 & $-8,40$ \\
\hline $0,25 \% \mathrm{wt}$ & 6.72 & 40.62 & 58,89 & $-9,75$ \\
\hline $0,125 \% \mathrm{wt}$ & 10.89 & 64.02 & 73,43 & $-12,50$ \\
\hline Average HTP value & & & $\mathbf{6 3 , 3 3}$ & $\mathbf{- 1 0 , 2 2}$ \\
\hline
\end{tabular}

a

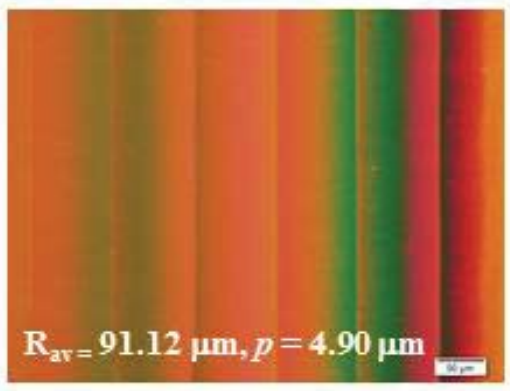

$\mathrm{b}$

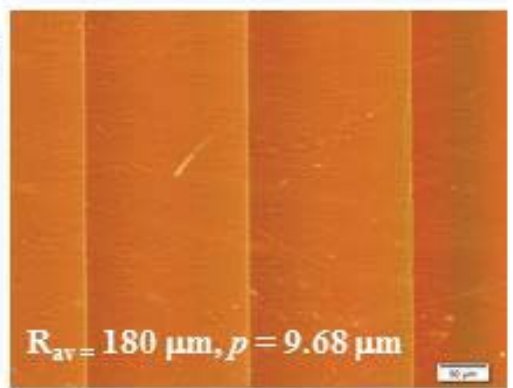

Figure 6.7 Polarized optical micrographs of Cano wedge cells of a) $0.5 \% w t, b) 0.25 w t \%$ of chiral dopant BB in DFNLC.; $\mathrm{R}_{\mathrm{av}}$ standing for average distance between Cano lines that implied a pitch $(p)$ of each cholesteric liquid crystal.

Table 6.2 HTP calculated for different concentrations of BB.

\begin{tabular}{|c|c|c|}
\hline Concentration of BB in DFNLC & $p_{i}(\mu \mathrm{m})$ & $\mathrm{HTP}_{\mathrm{BB}}(\mathrm{wt})$ \\
\hline $0.5 \% \mathrm{wt}$ & 4.90 & -38.15 \\
\hline $0.25 \% \mathrm{wt}$ & 9.68 & -39.74 \\
\hline Average HTP value & & $\mathbf{- 3 8 . 9 5}$ \\
\hline
\end{tabular}



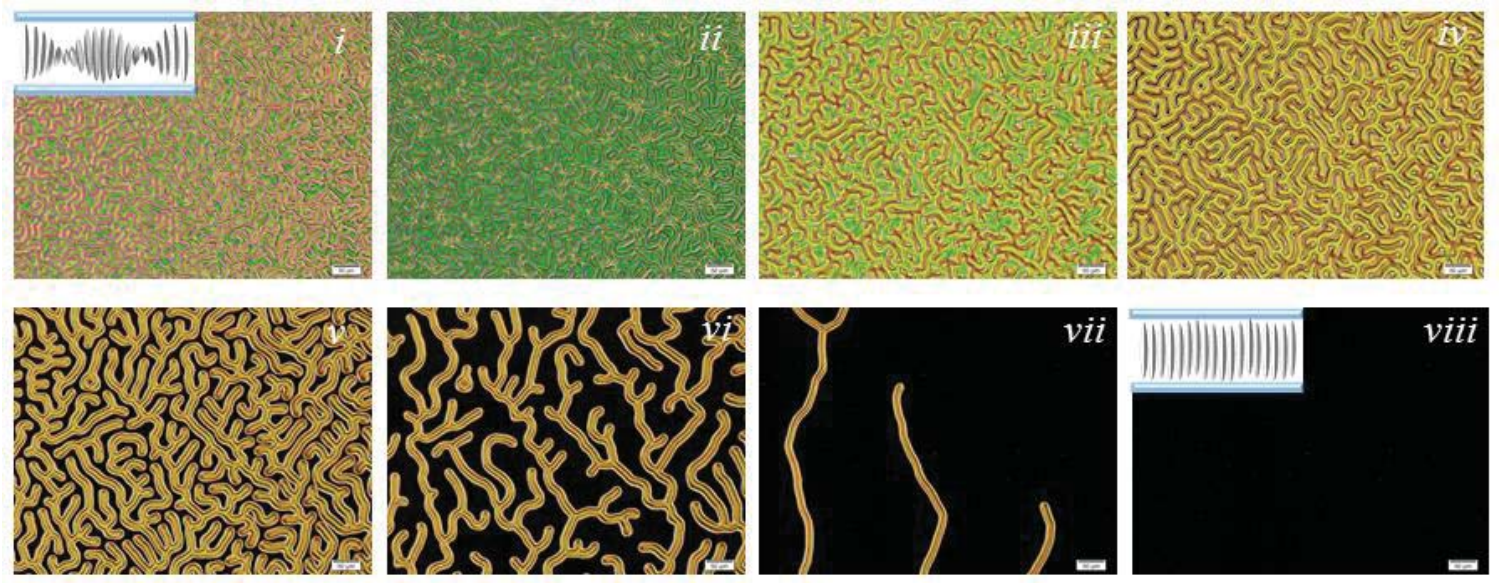

Figure 6.8 Thermal reversibility of dual-responsive film 1 under perpendicular boundary conditions. Scale bar: $50 \mu \mathrm{m}$.
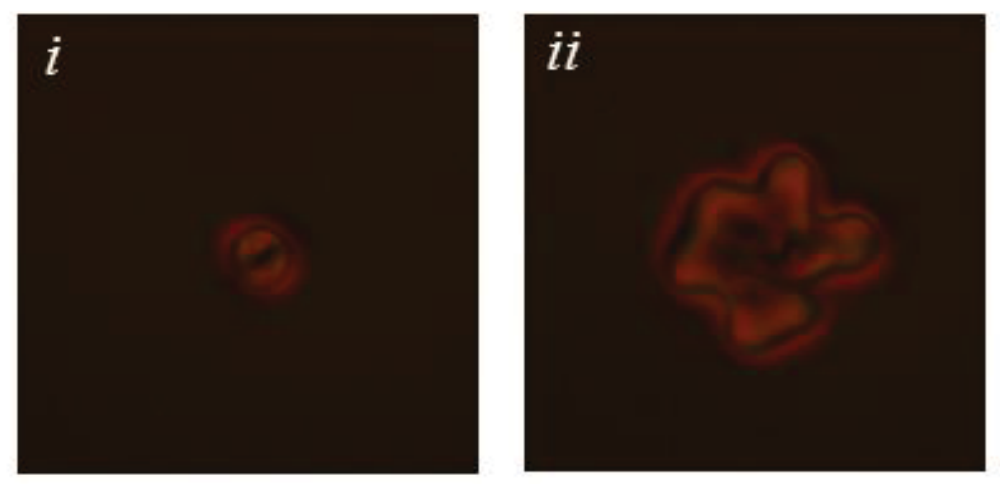

Figure 6.9 Creation of a defect by light and its manipulation in the homeotropic film $2(\mathrm{~d}=$ $10 \mu \mathrm{m})$ under pitch gradient condition by using split beams $\mathrm{B}_{1}=450 \mathrm{mkW}$ and $\mathrm{B}_{2}=0.9 \rightarrow$ $2.55 \mathrm{nW} ; i$ at initial state $(0.9 \mathrm{nW})$ and $i i$ at final state $(2.55 \mathrm{nW})$ upon increasing the power of $\mathrm{B}_{2}$.

\subsection{References}

1 Qi, M.; Lidorikis, E.; Rakich, P. T.; Johnson, S. G.; Joannopoulos, J. D.; Ippen, E. P.; Smith, H. I. Nature 2004, 429, 538.

2 Sun, C.-H.; Jiang, P. Nat Photon 2008, 2, 9.

3 Orlova, T.; Aßhoff, S. J.; Yamaguchi, T.; Katsonis, N.; Brasselet, E. Nat. Commun. 2015, 6 .

4 Broer, D. J. Nat. Mater. 2010, 9, 99.

5 Smalyukh, I. I.; Lansac, Y.; Clark, N. A.; Trivedi, R. P. Nat. Mater. 2010, 9, 139.

$6 \quad$ Oswald, P.; Baudry, J.; Pirkl, S. Phys. Rep. 2000, 337, 67. 
Towards dual responsive liquid crystal films

7 Ribiere, P.; Oswald, P. J. Phys. France 1990, 51, 1703.

$8 \quad$ Loussert, C.; Brasselet, E. Appl. Phys. Lett. 2014, 104, 051911.

9 Trushkevych, O.; Ackerman, P.; Crossland, W. A.; Smalyukh, I. I. Appl. Phys. Lett. 2010, 97, 201906.

10 Loussert, C.; Iamsaard, S.; Katsonis, N.; Brasselet, E. Adv. Mater. 2014, 26, 4242.

11 Paul J. Ackerman, Z. Q., and Ivan I. Smalyukh Phys. Rev. E 2012, 86, 021703.

12 Private communication with E. Brasselet, unpublished results.

13 Xianyu, H.; Wu, S.-T.; Lin, C.-L. Liq. Cryst. 2009, 36, 717.

14 Kosa, T.; Sukhomlinova, L.; Su, L.; Taheri, B.; White, T. J.; Bunning, T. J. Nature 2012, 485, 347.

15 Blinov, L. M. Structure and properties of liquid crystals; Springer 2011. 


\section{Summary}

Smart materials adapt to, rather than resist, changes to their environment. In Nature, a variety of smart materials in the biological system demonstrate unique and potential functions, which appear as norm in the design of smart synthetic materials. Liquid crystals are very useful in the engineering of smart synthetic materials because of their well-ordered and controllable structure, anisotropy, and high sensitivity to external stimuli. These properties promote the engineering of synthetic materials to display smart and potential functions comparative to those of biological materials. The research presented in this thesis describes strategies to develop new, functional and smart molecular materials by amplifying molecular motion, which is intrinsically limited to the nanoscale, up to the macroscopic level of functional materials. A special focus is given to molecular motion that is triggered by light as an external stimulus, and induces a change in chiral structure of cholesteric liquid crystals at molecular level, which is eventually amplified by cooperativity of the liquid crystals into either a mechanical or an optical output.

This work also provides insights into the mechanisms of amplification of molecular movement. While stabilizing the twisted organization of liquid crystals by in-situ polymerization, photo-induced molecular switching lead to disorder in the liquid crystal polymer network, which in turn created strain and was eventually transformed into mechanical motion at the macroscale (chapter 3 and 4), including both helical motion (chapter 3 and 4) and bending motion (chapter 4) which were shown to arise from a twisted geometry. Alternatively, starting from a ground state in which the twisting of a chiral liquid crystal is hampered, amplification of molecular motion was achieved by releasing this frustration, which was translated into original properties for this system (chapter 5 and 6). These investigations also point out that the amplification of molecular motion can be manipulated to reach different ranges, from the microscale (chapter 5 and 6) to the macroscale (chapter 3 and 4), by using an appropriate choice of irradiation with light (with either a local- or a spatial- focus). In addition to amplifying motion, we showed that chirality, an essential property of molecular matter, can be translated (e.g. chapter 3; molecular chirality indirectly amplified into macroscopic chiral shapes) or suppressed (e.g. chapter 5 
and 6; supramolecular chirality is suppressed using homeotropic confinement) at different scales. Changes of material design, such as architecture, chemical constitution, fabrication and controlled operation have impact on the amplification of molecular motion, molecular chirality as well as other intrinsic molecular properties to present variously at the level of functional materials (e.g. mechanical output in chapter 3 (helical motion) and chapter 4 (helical- and bending motion with slow shape relaxation), and optical output in chapter 5 (single stimuli-responsiveness) and chapter 6 (dual stimuli-responsiveness)). Notably, taking inspiration from the design of biological materials in nature can lead to effective strategies in engineering new, smart and sophisticated function of materials (e.g. chapter 3).

Overall, we have developed light-responsive liquid crystal polymer networks that are capable of performing light-induced helical motion and some of them also retain their lightinduced shape. The complex and versatile helix-based behavior they display suggests prospective use in applications such as soft robotics and microfluidic technology. Moreover, light-responsive chiral liquid crystals show original optical properties that could be used for the development of re-writable and photonic technologies. 


\section{Samenvatting}

Zogenoemde slimme (smart) materialen zijn niet alleen bestand tegen hun omgeving, maar passen zich zelfs aan aan veranderingen in die omgeving. In de natuur laten diverse slimme materialen in biologische systemen unieke functies zien - in de biologie zijn slimme materialen de norm, waardoor ze een waardevolle bron van inspiratie vormen. Vloeibare kristallen zijn erg nuttig bij het ontwerpen van slimme synthetische materialen vanwege hun goed geordende en controleerbare structuur, anisotropie en hoge gevoeligheid voor externe stimuli. Het onderzoek gepresenteerd in dit proefschrift beschrijft strategieën om nieuwe, functionele en slimme moleculaire materialen te ontwikkelen door moleculaire beweging intrinsiek beperkt tot de nanoschaal - uit te vergroten tot het macroscopische niveau van functionele materialen. Hierbij ligt de focus op moleculaire beweging die gestuurd wordt door licht als externe stimulus, wat een verandering op moleculaire schaal teweegbrengt en uiteindelijk uitvergroot wordt naar een mechanisch of optisch uitgangssignaal.

Dit werk geeft inzicht in de mechanismen van het uitvergroten van moleculaire beweging. Terwijl de gedraaide organisatie van vloeibare kristallen gestabiliseerd wordt door in-situ polymerisatie, leidt licht-geïnduceerde wisseling tot wanorde in het vloeibare kristal polymeernetwerk. Dit veroorzaakt lengteverandering en wordt uiteindelijk omgezet in mechanische beweging op de macroschaal (hoofdstuk 3 en 4), inclusief zowel spiraalvormige beweging (hoofdstuk 3 en 4) als buigende beweging (hoofdstuk 4), die beide veroorzaakt zijn door de gedraaide geometrie. Daarnaast is een uitgangstoestand gebruikt waarin de draaiing van een chiraal vloeibaar kristal werd gehinderd. Uitvergroten van moleculaire beweging is bereikt door deze beperking los te laten, wat zich vertaalde in originele eigenschappen voor dit systeem (hoofdstuk 5 en 6).

Deze onderzoeken laten ook zien dat het uitvergroten van moleculaire beweging gecontroleerd kan worden om verschillende lengteschalen te bereiken door een passende keuze te maken voor het bestralen met licht (met óf een lokale óf een ruimtelijke focus), van de microschaal (hoofdstuk 5 en 6) tot de macroschaal (hoofdstuk 3 en 4). Naast het uitvergroten van beweging laten we ook zien dat chiraliteit, een essentiële eigenschap van moleculaire materie, op verschillende lengteschalen vertaald kan worden (bijv. hoofdstuk 3 : 
moleculaire chiraliteit is indirect uitvergroot naar macroscopisch chirale vormen) of onderdrukt kan worden (bijv. hoofdstuk 5 en 6: supramoleculaire chiraliteit is onderdrukt door homeotrope insluiting). Veranderingen van materiaalontwerp, zoals architectuur, chemische samenstelling, fabricage en gecontroleerde werking, hebben invloed op de uitvergroting van moleculaire beweging, moleculaire chiraliteit en andere intrinsieke moleculaire eigenschappen die veelvoudig aanwezig zijn op het niveau van functionele materialen (bijv. een mechanisch uitgangssignaal in hoofdstuk 3 (spiraalvormige beweging) en hoofdstuk 4 (spiraalvormige en buigende beweging met langzame terugvorming) en een optisch uitgangssignaal in hoofdstuk 5 (enkele stimulus-responsiviteit) en hoofdstuk 6 (dubbele stimulus-responsiviteit)). Het is noemenswaardig dat inspiratie uit het ontwerp van biologische materialen in de natuur kan leiden tot effectieve strategieën in het ontwerpen van nieuwe, slimme en verfijnde functies van materialen (bijv. hoofdstuk 3).

Samenvattend hebben we licht-gestuurde vloeibaar kristal polymeernetwerken gemaakt die licht-geïnduceerde spiraalvormige bewegingen kunnen uitvoeren, waarbij sommige netwerken zelfs hun licht-geïnduceerde vorm behouden. Het complexe en veelzijdige spiraalgebaseerde gedrag dat ze laten zien suggereert toekomstig gebruik in toepassingen zoals zachte robotica en microfluïdische technologie. Licht-gestuurde chirale vloeibare kristallen laten bovendien originele optische eigenschappen zien die gebruikt zouden kunnen worden voor de ontwikkeling van herschrijfbare en fotonische technologieën. 


\section{Acknowledgements}

While completing this Ph.D. thesis, I have received immense support, help and many suggestions from many people. I would like to take this chance to express my sincere thanks to all of them.

I am especially grateful to my supervisor, Prof. Nathalie Katsonis, for providing me with an offer to study this Ph.D. with its many great opportunities for my life: experiencing life abroad, interesting research topics to learn, a chance to meet and work with kind, international and smart people, as well as joining English/ training courses and international conferences. My gratitude also for your research views and guidance throughout my study; it has allowed me to learn many new things and has developed my thinking. In particular, I have appreciated your unceasing motivation to push work forward and your talent for introducing and unfolding science in solid and fascinating ways that I have experienced from your correction on the draft of the thesis chapters.

I am also grateful to Prof. Jeroen Cornelissen, my co-promotor, for the opportunity of joining the BNT group, and for the advice and helpful attitude that you have provided throughout the study, specifically during BNT and individual work meetings.

Input from collaboration is also crucial to the completion of this thesis. I am thankful to Prof. Stephen Fletcher from Oxford University for suggesting key points and commenting constructively on the manuscript (in chapter 3 and 4) and also for the great experience of visiting your group, which broadened my synthetic view and knowledge. Many thanks to motivating members from his research group as well: Tiana for showing me the lab access and coaching me regarding flame dry reaction during my visit to the group in Oxford, Emanuela for synthesizing fluoro-azobenzene switches, and Elise for the first draft of nature protocol, together with our fruitful discussions during your visit to Enschede. Moreover, I am grateful to Dr. Etienne Brasselet from University of Bordeaux for creating the inventive project described in chapter 5 and 6 , collaborative works that have widened my knowledge of the physics side of liquid crystals.

I would like to express my gratitude to my committee members: Prof. D. J. Broer from Eindhoven University of Technology, Prof. Stephen Fletcher from Oxford University, 
Prof. Jurriaan Huskens and Dr. Mark Hempenius from University of Twente, for your comments on the concept thesis and for the honorable opportunity of having my Ph.D. defense under your consideration.

I am grateful to Prof. Jurriaan Huskens, Dr. Wim Verboom, Prof. Pascal Jonkheijm, Dr. Melissa Koay and Dr. Tibor Kudernac for raising intellectual questions and suggestions on my Ph.D. projects during the BNT \& MNF colloquium, which are useful to my study and have allowed me to learn and see what I missed. Many thanks to Tibor for creative experimental design and suggestions on work described in chapter 3, as well as helpful discussion on my project, especially during Nathalie's maternity leave.

My special thanks also go to the helpful technicians and staff members in our BNT and MNF group; Marcel for your help particularly with the specific tools used in my Ph.D. projects, for useful information about local searches and for funny conversations. Bothering you for help at your office, accompanied by small talk is enjoyable... (:) Regine, for help with chemicals and glassware, and your kindness. Richard, for an introduction to lab, UV spectrometer access as well as your help with fixing all my IT problems. Bianca, for introductions and information regarding NMR. Nicole and Izabel, for consistent help with many arrangements, especially with addressing my complicated administration and visa, and for always being kind even with the complicated issues.

Also, I am very pleased to show my thanks to workmates in BNT and MNF group for participation, help and company, which are very essential to both my work in this thesis and to my spirit;

Firstly, my big thanks to Sarah, a smart and charming lady, for sharing optical microscopy techniques, liquid crystal knowledge and great scientific advice. I am also very thankful for your sharing of fun stories with me and your cool way of thinking in our chats, your help from when I first came to Europe till now, your amity, and also all the moments we have shared in different activities and places, which have become good memories for me. ;) To my happy and amusing friend, Ben, I very much enjoyed working with you and things often go nicely in your company. Thank you a lot also for being my listener; your helpful advice and encouragement to both my work and my mind, together with all fun events you let me join and the particularly happy time I have had while sharing an apartment with you...Without the input ideas in the brainstorm meetings and contribution of you both 
(Sarah and Ben), the described work in chapter 3 of this thesis would not have been possible. Thank both of you again very much...:- Then, my special thanks goes to Tetiana, for your contribution to the work described in chapter 6; for your helpful discussion, your guidance in drafting chapter 6 and, eventually, correction over my first version of chapter 6 . Your strong competence in liquid crystals, deliberation and ability to always come up with wise explanations despite unexpected results are notable. It has been my luck to have had the chance to work and learn from you $\odot$. I also wish to thank to Federico very much, for your contribution and time for the protocol. Without you, I would not have been able to complete my study yet and, more importantly, it would have been such a pity to have missed working and talking with an independent and creative person with a strong background in science like you. My many thanks also go to Rianna for synthesizing the dopant used in Chapter 5 and 6 , your help including my house moving, cozy chats, and always being friendly to me. Janneke, thank you a lot for quick and effective Dutch summary and also for your friendliness.

In the next part, I would like to express my thanks to people who may not have directly participated in the work described in this thesis but have given meaningful help and encouragement to me. To Wilfried, funny and sincere friend, my special thanks to you for always cheering me up, primarily during the difficult time of my last year of study, your help with uses of Endnote, reminding me of my thesis timeline, as well as all the fun times at lunch and evening breaks with you. Although we have no overlapped projects, your help and goodwill has had a big effect on the success of this thesis...:- Tushar, our enjoyable and easy chats have been a relief and happiness to me. Apart from our funny talks, many thanks for sharing with me friendly advice about works in common and also the administrative issues of overseas students. I wish you a wonderful married life and enjoyment in your new career! To workmate girls who come from overseas, I would like to thank Meow (Supa), my fun and helpful paranymph, for amusing talks during our lunch time, your encouragement as well as the pleasant Thai cuisine you have shared with me, especially for saving me from starving during the thesis writing period...(-) To another kind and lively paranymph of mine, Rindia, I thank you for sharing with me your smart attitude regarding work, your advice in general, cheering me and also bringing fun and/or updated stories to our talks. Very special thanks to both of you for immediately accepting to be my paranymphs and all things you do 
for assisting me and my defense To Aijie, a sweet housemate and friend, many thanks for sharing with me polymer chemistry knowledge, and for the enjoyable activities you let me join, including meal celebrating, Chinese hot pot, going shopping using your car, your encouragement, as well as pleasant times at our house. Liuyi!!, a cheerful girl, thank you for our funny and girly talks, lovely dinners, and your words that always refresh me. I thank all you girls also for the great times during our meeting and small fun trip. I wish you all the best of luck in your Ph.D. work and future life!

My thanks goes to more workmates in our groups; Goodday (Tadatsugu) for giving me Japanese snacks while staying late in the lab, sharing me knowledge about kinetics of your spiropyran compounds and giving me inspiration through always keeping happy and smiling despite working hard. Alexis and He, for sharing with me your opinions of work, funny jokes and a good atmosphere at gatherings. Rajesh, for traditional Indian snacks, nice chats that make me awake when staying in the lab late, and your wishes. And finally, the real truth came!!!...to the most sucia girl ever!!!, Raquel, for bringing smile and happiness to me (particularly your amazing laugh, which can spread happiness to far kilometres...eiei). Sucia! You are a kind and very friendly person. Thank you also for help with the chapter format and your encouraging words. I wish you a great life ahead with Thomas!...:- While, to sucio , Leile, thank you for the advice on my presentation at the Munster symposium, the funny atmosphere together with the sucia and joyful talk. Tom, my kind former neighbour in the chicken farm, for your big help with translating Dutch document issues, particularly my unemployment benefit application, your clever advice when I had problems to proceed with such issues, and also your smiling chat. Jenny, my current and generous neighbor in new office, for helpful information about the thesis process, for lending me a book on scientific writing, for the nice occasions you invited me e.g. paper folding and cute chat. Bettina, for sharing tasty baking with me, also for nice occasions and cozy chats, especially for your kind explanations when I do not get the whole story in the chat or in the gathering because of my different cultural experiences and limitations in English. Emanuela, for lovely greetings and chats, kind explanations in gatherings and also your friendliness. Roberto for sharing interesting stories in the gatherings that often let me know things I never heard, and also for your amity. 
I also want to thank the following people in our groups, who are either my former or current colleagues for raising questions or suggestion on my works during the BNT and MNF \& BNT meeting, giving miscellaneous help to me in work or in group activities as well as friendship. (:): Tegar, Stan, Jenny, Mark, Robin, Christoph, Liulin, Chengfen, Maarten, Vijay, Melanie, Anne, Jealemy, Rik, Piotr, Martijn from BNT group and Laura, Gülistan, Andrea, Liang, Alexander, Rick, Jacopo, Daniele, Joao, Shirish, Andreas, Mark, Wouter, Maike, Jordi, Mudassir, Sven, Pieter, Carlo, Nicolai, Carmen, Jasper, Kim, Jiguang, Amal, Angle, Wies, Jens, Wei from MNF group.

Furthermore, I wish to express my deep gratitude to my former supervisor Prof. dr. Boonsong Kongkathip from Kasetsart university and Assistant Prof. dr. Tienthong Thongpanchang from Mahidol University, for providing me with valuable knowledge from your lectures and practical skills while performing thesis projects in the NPOS lab and TT lab, respectively. The value from these groups has greatly helped me go on in Ph.D. study. Many thanks to my former colleagues in the NPOS and TT labs, as well for training me in lab work and sharing your synthetic expertise with me. Importantly, my special thanks to (Arjarn) Tienthong Thongpanchang and (P') Nopporn Ruangsupapichat for bringing the Ph.D. opportunity to me and your support in helping me gain this Ph.D. position.

My thanks also to Thai students in Enschede, P' Lek, P' May, P' Pai, P' Siti, P' Tuk, P' Wow, P' Pin, Moew and $\mathbf{M}$ from UT and also Nong nong from Saxion, for general help, useful information, especially during my settlement in Enschede, fun sightseeing and also for traditional Thai meals.

To my friends from far, I am very thankful to Mai, Pang, Bu and Pik for fun stories, updates on life, as well as your cheer through our message chats.

Towards this end, I would like to express my deep thanks to my dear family; mom, dad, sister, brother and grandmother, for your unconditional love, care and constant support. Although, there are time shifts and a distance barrier between us, I especially thank you Mom! for consistent Skype talks, in which you listen to my update, give me mature advice on my life, and also encourage me. My special thanks also to Weerayut for your patience despite the barriers, companionship, together with happiness from our daily chats.

All the best to everyone 
Acknowledgements 


\section{About the author}

Supitchaya Iamsaard was born in Thailand on the $24^{\text {th }}$ of May 1985. She studied for a bachelor's degree in chemistry at Kasetsart University, Thailand and carried out a senior project in the group of Prof. dr. Boonsong Kongkathip. The project was on "Studies toward the synthesis of disaccharide, a part of anticancer agent, OSW-1". In 2010 she received a master's degree for her study in the field of organic chemistry at Mahidol University, Thailand. In this study she conducted thesis research on the topic of "Ether stabilized oxiranyl 'remote' anions" under the supervision of Assistant Prof. dr. Tienthong Thongpanchang. During the study, she gained a scholarship from the DPST (Development and Promotion of Science and Technology) under the Thai government for bachelor and master's level degrees and also a scholarship from PERCH-CIC (Center for Innovation in Chemistry: Post Graduate Education Research Program in Chemistry) for the master's level degree.

Since September 2011 she has been a Ph.D. candidate at the University of Twente under the supervision of Prof. dr. Nathalie Katsonis. The aim of the Ph.D. project is to synthesize photochromic molecules and exploit their molecular motion upon light activation to engineer light-responsive materials, based on cholesteric liquid crystals. The result of this project is described in this thesis. 


\section{List of publications}

1. S. Iamsaard, E. Villemin, F. Lancia, S.J. A $\beta$ hoff, S.P. Fletcher and N. Katsonis, Nature Protocols, submitted.

2. C. Loussert, S. Iamsaard, N. Katsonis, E. Brasselet, "Subnanowatt Opto-Molecular Generation of Localized Defects in Chiral Liquid Crystals" Adv. Mater. 2014, 26, $4242-4246$.

3. S. Iamsaard, S.J. Aßhoff, B. Matt, T. Kudernac, J.J.L.M. Cornelissen, S.P. Fletcher and N. Katsonis, "Conversion of light into macroscopic helical motion" Nat. Chem. 2014, 6, 229-235.

4. S.J. Aßhoff, S. Iamsaard, A. Bosco, J.J.L.M. Cornelissen, B.L. Feringa and N. Katsonis, "Time-programmed helix inversion in phototunable liquid crystals" Chem. Commun. 2013, 49, 4256-4258.

5. P. Saisaha, C. Nerungsi, S. Iamsaard, T. Thongpanchang, "Pyridine stabilized oxiranyl remote anions" Tetrahedron Lett. 2009, 50, 4217-4220. 

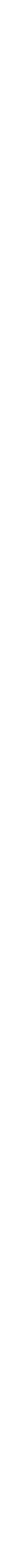